\title{
DISCRETE CARLEMAN ESTIMATES FOR ELLIPTIC OPERATORS AND UNIFORM CONTROLLABILITY OF SEMI-DISCRETIZED PARABOLIC EQUATIONS*
}

\author{
FRANCK BOYER ${ }^{\dagger \S}$, FLORENCE HUBERT ${ }^{\ddagger}$, AND JÉRÔME LE ROUSSEAU §
}

\begin{abstract}
We derive a semi-discrete two-dimensional elliptic global Carleman estimate, in which the usual large parameter is connected to the one-dimensional discretization step-size. The discretizations we address are some families of smoothly varying meshes. As a consequence of the Carleman estimate, we derive a partial spectral inequality of the form of that proved by G. Lebeau and L. Robbiano, in the case of a discrete elliptic operator in one dimension. Here, this inequality concerns the lower part of the discrete spectrum. The range of eigenvalues/eigenfunctions we treat is however quasi-optimal and represents a constant portion of the discrete spectrum. For the associated parabolic problem, we then obtain a uniform null controllability result for this lower part of the spectrum. Moreover, with the control function that we construct, the $L^{2}$ norm of the final state converges to zero super-algebraically as the step-size of the discretization goes to zero. A relaxed observability estimate is then deduced.
\end{abstract}

Key words. Elliptic operator, discrete Carleman estimate, spectral inequality, parabolic equation, semi-discrete scheme, uniform controllability / observability.

AMS subject classifications. 35K05 - 65M06 - 93B05 - 93B07 - $93 \mathrm{~B} 40$

1. Introduction and settings. Let $\Omega, \omega$ be connected non-empty bounded open subsets of $\mathbb{R}^{n}$ with $\omega \Subset \Omega$. We consider the following parabolic problem in $(0, T) \times \Omega$, with $T>0$,

$$
\partial_{t} y-\nabla_{x} \cdot\left(\gamma \nabla_{x} y\right)=\mathbf{1}_{\omega} v \text { in }(0, T) \times \Omega,\left.\quad y\right|_{\partial \Omega}=0, \quad \text { and }\left.y\right|_{t=0}=y_{0},
$$

where the diffusion coefficient $\gamma=\gamma(x)>0$ satisfies

$$
\operatorname{reg}(\gamma) \stackrel{\text { def }}{=} \sup _{x \in \Omega}\left(\gamma(x)+\frac{1}{\gamma(x)}+\left|\nabla_{x} \gamma(x)\right|\right)<+\infty .
$$

G. Lebeau and L. Robbiano proved in [LR95] the null controllability of system (1.1), i.e., for all $y_{0} \in L^{2}(\Omega)$, there exists $v \in L^{2}((0, T) \times \Omega)$, such that $y(T)=0$ and $\|v\|_{L^{2}((0, T) \times \Omega)} \leq C\left|y_{0}\right|_{L^{2}(\Omega)}$, where $C>0$ only depends on $\Omega, \omega, \gamma$ and $T$. They in fact constructed the control function $v$ semi-explicitly. This construction is based on the following spectral inequality.

TheOREM 1.1 ([LR95, JL99, LZ98a]). Let $\left(\phi_{k}\right)_{k \in \mathbb{N}^{*}}$ be a set of $L^{2}(\Omega)$-orthonormal eigenfunctions of the operator $\mathcal{A}:=-\nabla_{x} \cdot\left(\gamma \nabla_{x}\right)$ with homogeneous Dirichlet boundary conditions, and $\left(\mu_{k}\right)_{k \in \mathbb{N}^{*}}$ be the set of the associated eigenvalues (with finite multiplicities) sorted in a non-decreasing sequence. There exists $C>0$ such that for all

\footnotetext{
Date: November 4, 2009

*The three authors were partially supported by l'Agence Nationale de la Recherche under grant ANR-07-JCJC-0139-01.

†Université Paul Cézanne (fboyer@latp.univ-mrs.fr).

¥Université de Provence (fhubert@latp. univ-mrs.fr).

$\S$ Laboratoire d'Analyse Topologie Probabilités (LATP), CNRS UMR 6632, Aix-Marseille Universit, 39 rue F. Joliot-Curie, 13453 Marseille cedex 13, France.

I Université de Provence. Current address: Laboratoire Mathématiques et Applications, Physique Mathématique d'Orléans, CNRS UMR 6628, Université d'Orléans (jlr@univ-orleans.fr). Part of this work was carried out where the third author was on a research leave at Laboratoire POEMS, INRIA Paris-Rocquencourt/ENSTA, CNRS UMR 2706, France.
} 


$$
\begin{aligned}
& \mu \geq 0 \text { and for all }\left(\alpha_{k}\right)_{k \in \mathbb{N}^{*}} \subset \mathbb{C} \\
& \qquad \sum_{\mu_{k} \leq \mu}\left|\alpha_{k}\right|^{2}=\int_{\Omega}\left|\sum_{\mu_{k} \leq \mu} \alpha_{k} \phi_{k}(x)\right|^{2} d x \leq C e^{C \sqrt{\mu}} \int_{\omega}\left|\sum_{\mu_{k} \leq \mu} \alpha_{k} \phi_{k}(x)\right|^{2} d x .
\end{aligned}
$$

The proof of this result relied on local Carleman estimates for the augmented elliptic operator $-\partial_{t}^{2}+\mathcal{A}$ in $\left(0, T_{*}\right) \times \Omega$, for some $T_{*}>0$, where $t$ is an additional variable.

This article provides similar results, i.e., elliptic Carleman estimates, a LebeauRobbiano-type spectral inequality, and controllability result, in the case of a spatial discretization of the parabolic operator in (1.1).

To our knowledge, in the discrete case, the only positive uniform null controllability result is the one in [LZ98b] concerning the case of a boundary control in 1D, with a constant diffusion coefficient $\gamma$ and for a constant step size finite-difference discretization. In two dimensions, again for finite differences, there is however a counter-example to the null and approximate controllabilities for uniform grids on a square domain for distributed or boundary control (see [Zua06]).

On the one hand, the proof of the result of [LZ98b] relies on a decomposition along a basis of explicit eigenfunctions of the finite-difference approximation of $\mathcal{A}$ in one dimension, thus requiring the diffusion coefficient $\gamma$ and the step size to be constant. On the other hand, the counter-example provided in [Zua06], exploits an explicit eigenfunction of $\mathcal{A}$ in two dimensions that is solely localized on the diagonal of the square domain. It naturally follows that the control region (distributed control or boundary control) would have to meet the diagonal of the domain for the null or approximate controllabilities to hold.

In this article, we concentrate on distributed control. The case of a boundary control can then be obtained following a domain extension method (see e.g. [FI96]). To address non uniform discretizations and non constant diffusion coefficients, we propose to base our analysis on discrete global Carleman estimates. As a first step, in this article, for the sake of exposition, we restrict our analysis of semi-discrete parabolic operators to one dimension in space. However, the proof of such Carleman estimates does not effectively rely on the space dimension. As a consequence, we cannot expect to obtain any uniform controllability result for the full spectrum with this method, even in one dimension, because of the counter-example in higher dimension.

In [Zua06, Zhe08], the derivation of discrete Carleman estimates was proposed as a challenging research problem. In fact, in the course of the proof of such estimates, the Carleman large parameter $s$ has to be connected to the mesh size $h$ : we obtain a condition of the form $s h \leq \varepsilon_{0}$, with $\varepsilon_{0}=\varepsilon_{0}(\Omega, \omega, \gamma)$. This kind of condition cannot be avoided: without such a restriction we would be able to achieve a Lebeau-Robbiano spectral inequality for the full spectrum of the discrete operator. Yet, such a result does not hold (see Remark 1.3 below). Note that an earlier attempt at deriving discrete Carleman estimates can be found in [KS91]. The result presented in [KS91] cannot be used here as the condition imposed by these authors on the discretization step size, in connection to the large Carleman parameter, is too strong.

Here, the condition $s h \leq \varepsilon_{0}$ in the Carleman estimate only yields a partial LebeauRobbiano spectral inequality for the lower part of the spectrum. By "lower part" we actually mean a constant portion of the discrete spectrum (see Remark 1.5 below). In particular, the Lebeau-Robbiano inequality for the full spectrum of the differential operator $\mathcal{A}$ can be recovered when $h$ goes to zero.

As far as the controllability result in the semi-discrete case is concerned, we 
consider the following system,

$$
\partial_{t} y_{h}+\mathcal{A}^{\mathfrak{M}} y_{h}=\mathbf{1}_{\omega} v_{h},\left.\quad y_{h}\right|_{\partial \Omega}=0,\left.\quad y\right|_{t=0}=y_{0}^{h},
$$

where $\mathcal{A}^{\mathfrak{M}}$ is a discrete approximation of $\mathcal{A}$ for a mesh $\mathfrak{M}$ with step-size $h$ to be precisely introduced below. We prove that there exists a control function $v_{h}$, with $\left\|v_{h}\right\|_{L^{2}((0, T) \times \omega)} \leq C\left|y_{0}^{h}\right|_{L^{2}(\Omega)}, C>0$ independent of $h$, such that the frequencies of the controlled solution $y_{h}$ associated to the lower part of the spectrum vanish at the final time $T$. We furthermore prove that

$$
\left|y_{h}(T)\right|_{L^{2}(\Omega)} \leq C e^{-C / h^{2}}\left|y_{0}^{h}\right|_{L^{2}(\Omega)} .
$$

This should not be considered as an approximate controllability result and should rather be compared with the result obtained in [LT06], where they proved (in a somewhat more general framework) a result of the form (1.3) with $e^{-C / h^{2}}$ replaced by $h^{\alpha}$, for some explicit exponent $\alpha>0$. See also the observability estimate (1.10) below. Note that in the sequel we shall drop the subscript $h$, in the case of discrete function, as in $y_{h}$ or $v_{h}$, for the sake of concision.

As mentioned above, we chose to restrict ourselves in one space dimension since additional technicalities are needed for the multidimensional case. This issue will be developed in future work [BHL09a]. With the discrete partial Lebeau-Robbiano inequality we prove here, the full discrete problem can also be addressed [BHL09b, BHL09c].

A challenging question lays in the derivation of uniform discrete parabolic global Carleman estimates. In the continuous case, global parabolic Carleman estimates were introduced in [FI96] and they in particular lead to the null controllability of linear and semi-linear parabolic equations [Bar00, FCZ00]. Like in the elliptic case that we treat here, we cannot hope to obtain such estimates, in the discrete parabolic case, with an arbitrary large parameter.

1.1. Discrete settings. As mentioned above we restrict our analysis of semidiscrete parabolic operators to one dimension in space. Let us consider the elliptic operator on $\Omega=(a, b)$ given by $\mathcal{A}=-\partial_{x}\left(\gamma \partial_{x}\right)$ with homogeneous Dirichlet boundary conditions and $\gamma$ satisfying (1.2).

We introduce finite difference approximations of the operator $\mathcal{A}$. Let $a=x_{0}<$ $x_{1}<\cdots<x_{N}<x_{N+1}=b$, see Figure 1.1. We refer to this discretization as to the primal mesh $\mathfrak{M}:=\left\{x_{i} ; i=1, \ldots, N\right\}$. We set $|\mathfrak{M}|:=N$. We set $h_{i+\frac{1}{2}}=x_{i+1}-x_{i}$ and $x_{i+\frac{1}{2}}=\left(x_{i+1}+x_{i}\right) / 2, i=0, \ldots, N$, and $h=\max _{0 \leq i \leq N} h_{i+\frac{1}{2}}$. We call $\overline{\mathfrak{M}}:=$ $\left\{x_{i+\frac{1}{2}} ; i=0, \ldots, N\right\}$ the dual mesh and we set $h_{i}=x_{i+\frac{1}{2}}-x_{i-\frac{1}{2}}=\left(h_{i+\frac{1}{2}}+h_{i-\frac{1}{2}}\right) / 2$, $i=1, \ldots, N$.

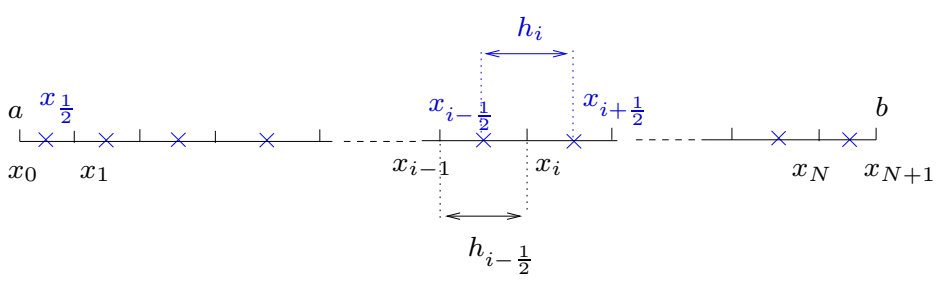

FIG. 1.1. Notation for the mesh geometry 
In the present article, we shall only consider some families of regular non uniform meshes, that will be precisely defined in Section 1.2. Note that the extension of our results to more general mesh families does not seem to be straightforward.

We denote by $\mathbb{C}^{\mathfrak{M}}$ and $\mathbb{C}^{\overline{\mathfrak{M}}}$ the sets of discrete functions defined on $\mathfrak{M}$ and $\overline{\mathfrak{M}}$ respectively. If $u \in \mathbb{C}^{\mathfrak{M}}$ (resp. $\mathbb{C}^{\overline{\mathfrak{M}}}$ ), we denote by $u_{i}$ (resp. $u_{i+\frac{1}{2}}$ ) its value corresponding to $x_{i}$ (resp. $\left.x_{i+\frac{1}{2}}\right)$. For $u \in \mathbb{C}^{\mathfrak{M}}$ we define

$$
u^{\mathfrak{M}}=\sum_{i=1}^{N} \mathbf{1}_{\left[x_{i-\frac{1}{2}}, x_{i+\frac{1}{2}}\right]} u_{i} \in L^{\infty}(\Omega)
$$

Since no confusion is possible, by abuse of notation we shall often write $u$ in place of $u^{\mathfrak{M}}$. For $u \in \mathbb{C}^{\mathfrak{M}}$ we define $\int_{\Omega} u:=\int_{\Omega} u^{\mathfrak{M}}(x) d x=\sum_{i=1}^{N} h_{i} u_{i}$.

For $u \in \mathbb{C}^{\bar{M}}$ we define

$$
u^{\overline{\mathfrak{M}}}=\sum_{i=0}^{N} \mathbf{1}_{\left[x_{i}, x_{i+1}\right]} u_{i+\frac{1}{2}} .
$$

As above, for $u \in \mathbb{C}^{\overline{\mathfrak{M}}}$, we define $\int_{\Omega} u:=\int_{\Omega} u^{\overline{\mathfrak{M}}}(x) d x=\sum_{i=0}^{N} h_{i+\frac{1}{2}} u_{i+\frac{1}{2}}$. Similarly, with $Q=(0, T) \times \Omega$, and $u(t)$ in $\mathbb{C}^{\mathfrak{M}}$ or $\mathbb{C}^{\overline{\mathfrak{M}}}$ for all $t \in(0, T)$, we shall write $\iint_{Q} u d t=$ $\int_{\mathbb{C}^{\frac{0}{\mathfrak{M}}}}^{T} \int_{\Omega} u(t) d t$. In particular we define the following $L^{2}$ inner product on $\mathbb{C}^{\mathfrak{M}}$ (resp.

$$
(u, v)_{L^{2}}=\int_{\Omega} u^{\mathfrak{M}}(x)\left(v^{\mathfrak{M}}(x)\right)^{*} d x, \quad \text { resp. } \quad(u, v)_{L^{2}}=\int_{\Omega} u^{\overline{\mathfrak{M}}}(x)\left(v^{\overline{\mathfrak{M}}}(x)\right)^{*} d x .
$$

For some $u \in \mathbb{C}^{\mathfrak{M}}$, we shall need to associate boundary conditions $u^{\partial \mathfrak{M}}=\left\{u_{0}, u_{N+1}\right\}$. The set of such extended discrete functions is denoted by $\mathbb{C}^{\mathfrak{M} \cup \partial \mathfrak{M}}$. Homogeneous Dirichlet boundary conditions then consist in the choice $u_{0}=u_{N+1}=0$, in short $u^{\partial \mathfrak{M}}=0$. We can now define translation operators $\tau^{ \pm}$, a difference operator $D$ and an averaging operator as the maps $\mathbb{C}^{\mathfrak{M} \cup \partial \mathfrak{M}} \rightarrow \mathbb{C}^{\overline{\mathfrak{M}}}$ given by

$$
\begin{array}{ll}
\left(\tau^{+} u\right)_{i+\frac{1}{2}}:=u_{i+1}, \quad\left(\tau^{-} u\right)_{i+\frac{1}{2}}:=u_{i}, & i=0, \ldots, N \\
(D u)_{i+\frac{1}{2}}:=\frac{1}{h_{i+\frac{1}{2}}}\left(\tau^{+} u-\tau^{-} u\right)_{i+\frac{1}{2}}, & \tilde{u}:=\frac{1}{2}\left(\tau^{+}+\tau^{-}\right) u
\end{array}
$$

We also define, on the dual mesh, translations operators $\tau^{ \pm}$, a difference operator $\bar{D}$ and an averaging operator as the maps $\mathbb{C}^{\bar{M}} \rightarrow \mathbb{C}^{\mathfrak{M}}$ given by

$$
\begin{aligned}
& \left(\tau^{+} u\right)_{i}:=u_{i+\frac{1}{2}}, \quad\left(\tau^{-} u\right)_{i}:=u_{i-\frac{1}{2}}, \quad i=1, \ldots, N \\
& (\bar{D} u)_{i}:=\frac{1}{h_{i}}\left(\tau^{+} u-\tau^{-} u\right)_{i}, \quad \bar{u}:=\frac{1}{2}\left(\tau^{+}+\tau^{-}\right) u
\end{aligned}
$$

Note that there is no need for boundary conditions here.

A continuous function $f$ defined in a neighborhood of $\bar{\Omega}$ can be sampled on the primal mesh $f^{\mathfrak{M}}=\left\{f\left(x_{1}\right), \ldots, f\left(x_{N}\right)\right\}$ which we identify to

$$
f^{\mathfrak{M}}=\sum_{i=1}^{N} \mathbf{1}_{\left[x_{i-\frac{1}{2}}, x_{i+\frac{1}{2}}\right]} f_{i}, \quad f_{i}=f\left(x_{i}\right), \quad i=1, \ldots, N
$$

We also set

$$
f^{\partial \mathfrak{M}}=\left\{f\left(x_{0}\right), f\left(x_{N+1}\right)\right\}, \quad f^{\mathfrak{M} \cup \partial \mathfrak{M}}=\left\{f\left(x_{0}\right), f\left(x_{1}\right), \ldots, f\left(x_{N}\right), f\left(x_{N+1}\right)\right\} .
$$




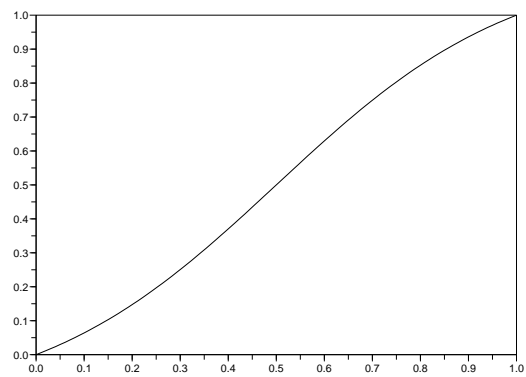

FIG. 1.2. Some non-uniform meshes for $N=10,20,40$ (left) and the corresponding map $\vartheta$ (right).

The function $f$ can also be sampled on the dual mesh $f^{\bar{M}}=\left\{f\left(x_{\frac{1}{2}}\right), \ldots, f\left(x_{N+\frac{1}{2}}\right)\right\}$ which we identify to

$$
f^{\bar{M}}=\sum_{i=0}^{N} \mathbf{1}_{\left[x_{i}, x_{i+1}\right]} f_{i+\frac{1}{2}}, \quad f_{i+\frac{1}{2}}=f\left(x_{i+\frac{1}{2}}\right), \quad i=0, \ldots, N .
$$

In the sequel, we shall often use $f$ for both the continuous function and its discretization on the primal mesh, i.e., $f^{\mathfrak{M} \cup \partial \mathfrak{M}}$. We shall write $f_{d}$ for the sampling $f^{\bar{M}}$ of $f$ on the dual mesh. In fact we shall write $D f:=D f^{\mathfrak{M} \cup \partial \mathfrak{M}}$ and $\bar{D} f_{d}:=\bar{D} f^{\bar{M}}$, with similar conventions for compositions of the discrete operators we defined above. See also Remark 3.1 for conventions concerning the action of discrete operators on continuous functions.

Throughout the article, a volume norm, i.e., over an open subset of $Q=(0, T) \times \Omega$, will be denoted by $\|$.$\| ; a surface norm will be denoted by |.|. Note that we shall use$ the same norm signs for continuous, semi-discrete and discrete norms over volumes and surfaces. For a semi-discrete function $u$ on $Q$, i.e., with $u(t) \in \mathbb{C}^{\mathfrak{M}}$ or $\mathbb{C}^{\bar{M}}$ for all $t \in(0, T)$, we thus set $\|u\|_{L^{2}(Q)}^{2}=\int_{0}^{T} \int_{\Omega}|u(t)|^{2} d t$.

1.2. Regular families of non-uniform meshes. In this paper, we address non uniform meshes that are obtained as the smooth image of an uniform grid.

More precisely, let $\left.\Omega_{0}=\right] 0,1[$ and let $\vartheta: \mathbb{R} \mapsto \mathbb{R}$ be an increasing map such that

$$
\vartheta \in \mathscr{C}^{\infty}, \quad \vartheta\left(\overline{\Omega_{0}}\right)=\bar{\Omega}, \quad \inf _{\overline{\Omega_{0}}} \vartheta^{\prime}>0 .
$$

Given an integer $N$, let $\mathfrak{M}_{0}=\left(i h^{\star}\right)_{1 \leq i \leq N}$, with $h^{\star}=\frac{1}{N+1}$ be a uniform mesh of $\Omega_{0}$ and $\overline{\mathfrak{M}_{0}}$ the dual mesh. We define a non-uniform mesh $\mathfrak{M}$ of $\Omega$ as the image of $\mathfrak{M}_{0}$ by the map $\vartheta$, setting

$$
x_{i}=\vartheta\left(i h^{\star}\right), \quad \forall i \in\{0, \ldots, N+1\} .
$$

The dual mesh $\overline{\mathfrak{M}}$, and the general notation are those of the previous section. We give in Figure 1.2 an example of such a family of non-uniform meshes and the map $\vartheta$ that we used to construct those meshes. 
1.3. Statement of the main results. With the notation we have introduced, a consistent finite difference approximation of $\mathcal{A} u$ with homogeneous boundary conditions is $\mathcal{A}^{\mathfrak{M}} u=-\bar{D}\left(\gamma_{d} D u\right)$ for $u \in \mathbb{C}^{\mathfrak{M} \cup \partial \mathfrak{M}}$ satisfying $u^{\partial \mathfrak{M}}=0$. Recall that $\gamma_{d}$ is the sampling of the given continuous diffusion coefficient $\gamma$ on the dual mesh $\overline{\mathfrak{M}}$, so that for any $u \in \mathbb{C}^{\mathfrak{M} \cup \partial \mathfrak{M}}$ we have

$$
\left(\mathcal{A}^{\mathfrak{M}} u\right)_{i}=-\frac{\gamma\left(x_{i+\frac{1}{2}}\right) \frac{u_{i+1}-u_{i}}{h_{i+\frac{1}{2}}}-\gamma\left(x_{i-\frac{1}{2}}\right) \frac{u_{i}-u_{i-1}}{h_{i-\frac{1}{2}}}}{h_{i}}, \quad i=1, \ldots, N .
$$

Note however that other consistent choices of discretization of $\gamma$ are possible, such as $\tilde{\gamma}$, i.e. the averaging on the dual mesh of the sampling of $\gamma$ on the primal mesh.

REMARK 1.2. Note that the discretization we have introduced can also be viewed as a finite volume approximation of the problem on the dual mesh.

For a suitable weight function $\varphi$, the announced semi-discrete Carleman estimate for the operator $P^{\mathfrak{M}}=-\partial_{t}^{2}+\mathcal{A}^{\mathfrak{M}}$ on $\left(0, T_{*}\right) \times \Omega$, for the non-uniform meshes we consider, is of the form

$$
\begin{gathered}
s^{3}\left\|e^{s \varphi} u\right\|_{L^{2}(Q)}^{2}+s\left\|e^{s \varphi} \partial_{t} u\right\|_{L^{2}(Q)}^{2}+s\left\|e^{s \varphi_{d}} D u\right\|_{L^{2}(Q)}^{2}+s\left|e^{s \varphi(0, .)} \partial_{t} u(0, .)\right|_{L^{2}(\Omega)}^{2} \\
+s e^{2 s \varphi\left(T_{*}\right)}\left|\partial_{t} u\left(T_{*}, .\right)\right|_{L^{2}(\Omega)}^{2}+s^{3} e^{2 s \varphi\left(T_{*}\right)}\left|u\left(T_{*}, .\right)\right|_{L^{2}(\Omega)}^{2} \\
\leq C\left(\left\|e^{s \varphi} P^{\mathfrak{M}} u\right\|_{L^{2}(Q)}^{2}+s e^{2 s \varphi_{d}\left(T_{*}\right)}\left|D u\left(T_{*}, .\right)\right|_{L^{2}(\Omega)}^{2}+s\left|e^{s \varphi(0, .)} \partial_{t} u(0, .)\right|_{L^{2}(\omega)}^{2}\right)
\end{gathered}
$$

for any $s \geq s_{0}$, and any $h \leq h_{0}$ such that $s h \leq \varepsilon_{0}$, and any $u$ satisfying $\left.u\right|_{\{0\} \times \Omega}=0$, $\left.u\right|_{\left(0, T_{*}\right) \times \partial \Omega}=0$, where $s_{0}, h_{0}$ and $\varepsilon_{0}$ only depend on the data (see Theorem 5.5). The proof of this estimate will be first carried out for uniform meshes, and then adapted to the case of non-uniform meshes we introduced in Section 1.2.

Note that the discrete operator $\mathcal{A}^{\mathfrak{M}}$ is selfadjoint with respect to the $L^{2}$ inner product on $\mathbb{C}^{\mathfrak{M}}$ introduced in (1.4). We denote by $\phi^{\mathfrak{M}}$ a set of discrete $L^{2}$ orthonormal eigenfunctions, $\phi_{j} \in \mathbb{C}^{\mathfrak{M}}, 1 \leq j \leq|\mathfrak{M}|$, of the operator $\mathcal{A}^{\mathfrak{M}}$, and by $\mu^{\mathfrak{M}}=\left\{\mu_{j}, 1 \leq\right.$ $j \leq|\mathfrak{M}|\}$ the set of the associated eigenvalues sorted in a non-decreasing sequence.

The announced partial Lebeau-Robbiano spectral inequality for the lower part of the spectrum reads

$$
\sum_{\substack{\mu_{k} \in \mu \mathfrak{M} \\ \mu_{k} \leq \mu}}\left|\alpha_{k}\right|^{2}=\int_{\Omega}\left|\sum_{\substack{\mu_{k} \in \mu \mathfrak{M} \\ \mu_{k} \leq \mu}} \alpha_{k} \phi_{k}\right|^{2} \leq C e^{C \sqrt{\mu}} \int_{\omega}\left|\sum_{\substack{\mu_{k} \in \mu \mathfrak{M} \\ \mu_{k} \leq \mu}} \alpha_{k} \phi_{k}\right|^{2}, \quad \forall\left(\alpha_{k}\right)_{1 \leq k \leq|\mathfrak{M}|} \subset \mathbb{C} .
$$

for $C>0$ only depending on $(\Omega, \omega, \gamma, \vartheta)$ and for $\mu h^{2}$ and $h$ sufficiently small. (see Theorem 6.1 for details).

REMARK 1.3. The inequality we have obtained only concerns a constant portion of the discrete spectrum. It is however quasi-optimal by the following argument. Observe indeed that the map

$$
\left(\alpha_{k}\right)_{1 \leq k \leq M} \in \mathbb{C}^{M} \mapsto\left(\sum_{1 \leq k \leq M} \alpha_{k} \phi_{k}\left(x_{j}\right)\right)_{x_{j} \in \omega} \in \mathbb{C}^{N_{\omega}}
$$

where $N_{\omega}=\#(\mathfrak{M} \cap \omega)$, is never injective if $M>N_{\omega}$. The maximal number of eigenfunctions we could possibly have in such an inequality is then of the order of $\frac{|\omega| N \mid}{|\Omega|}$. 
Since we can prove the asymptotic behavior $\mu_{k} \sim C k^{2}$, we are clearly restricted to the condition $\mu h^{2} \leq C \frac{|\omega|^{2}}{|\Omega|^{2}}$. We show here that the discrete Lebeau-Robbiano inequality holds for $\mu h^{2} \leq \varepsilon_{0}$ but we do not know if the $\varepsilon_{0}$ we obtain is optimal.

We introduce the following finite dimensional spaces

$$
E_{j}=\operatorname{Span}\left\{\phi_{k} ; 1 \leq \mu_{k} \leq 2^{2 j}\right\} \subset \mathbb{C}^{\mathfrak{M}}, \quad j \in \mathbb{N},
$$

and denote by $\Pi_{E_{j}}$ the $L^{2}$ orthogonal projection onto $E_{j}$. The controllability result we can deduce from the above results is the following.

Theorem 1.4. Let $T>0$ and $\vartheta$ satisfying (1.7). There exist $h_{0}>0, C_{T}>0$ and $C_{1}, C_{2}, C_{3}>0$ such that for all meshes $\mathfrak{M}$ defined by (1.8), with $0<h \leq h_{0}$, and all initial data $y_{0} \in \mathbb{C}^{\mathfrak{M}}$, there exists a semi-discrete control function $v$ such that the solution to

$$
\partial_{t} y-\bar{D}\left(\gamma_{d} D y\right)=\mathbf{1}_{\omega} v, \quad y^{\partial \mathfrak{M}}=0,\left.\quad y\right|_{t=0}=y_{0} .
$$

satisfies $\Pi_{E_{j} \mathfrak{m}} y(T)=0$, for $j^{\mathfrak{M}}=\max \left\{j ; 2^{2 j} \leq C_{1} / h^{2}\right\}$, with $\|v\|_{L^{2}(Q)} \leq C_{T}\left|y_{0}\right|_{L^{2}(\Omega)}$ and furthermore $|y(T)|_{L^{2}(\Omega)} \leq C_{2} e^{-C_{3} / h^{2}}\left|y_{0}\right|_{L^{2}(\Omega)}$.

The different constants $h_{0}, C_{j}, j=1,2,3$, appearing in the statement of the theorem will be made more explicit in the main text.

REMARK 1.5. Here the highest mode we are able to control uniformly satisfies $\mu_{k} \leq \varepsilon_{1} / h^{2}$. In fact for some $d_{1}>0$ and $d_{2}>0$, for all $1 \leq k \leq N$ we have $d_{1} k^{2} \leq \mu_{k} \leq d_{2} k^{2}$. It follows that we can treat any mode that satisfies $d_{2} k^{2} \leq$ $\varepsilon_{1} / h^{2} \leq C N^{2}$, or rather $k \leq C^{\prime} N$. The result of Theorem 1.4 thus states the null controllability of a constant portion of the discrete spectrum. Furthermore, note that for $h$ sufficiently small the error made for the remainder of the spectrum goes to zero super-algebraically.

The (relaxed) observability estimate we then obtain is of the form

$$
|q(0)|_{L^{2}(\Omega)} \leq C_{T}\left(\int_{0}^{T} \int_{\omega}|q(t)|^{2} d t\right)^{\frac{1}{2}}+C e^{-C / h^{2}}|q(T)|_{L^{2}(\Omega)} .
$$

for any $q$ solution to the adjoint system of system (1.9) (see Corollary 7.5 for details).

1.4. Outline. In Section 2, in the continuous case, we present an alternative method to prove the Lebeau-Robbiano spectral inequality. A large part of the article is dedicated to the extension of this approach to the discrete case. In Section 3 we have gathered preliminary discrete calculus results. To ease the reading most of the proofs have been placed in Appendix A. Section 4 is devoted to the proof of the semi-discrete elliptic Carleman estimate for uniform meshes. Again, to ease the reading, a large number of proofs of intermediate estimates have been placed in Appendix B. This result is then extended to non-uniform meshes in Section 5. In Section 6, with such a Carleman estimate at hand, we derive a partial discrete Lebeau-Robbiano spectral inequality. Finally, in Section 7, as an application, we prove the controllability result of Theorem 1.4. 
1.5. Additional notation. We shall denote by $z^{*}$ the complex conjugate of $z \in \mathbb{C}$. In the sequel, $C$ will denote a generic constant independent of $h$, whose value may change from line to line. As usual, we shall denote by $\mathcal{O}(1)$ a bounded function. We shall denote by $\mathcal{O}_{\mu}(1)$ a function that depends on a parameter $\mu$ and is bounded once $\mu$ is fixed. The notation $C_{\mu}$ will denote a constant whose value depends on the parameter $\mu$.

We sometimes use multi-indices. We say that $\alpha$ is a multi-index if $\alpha=\left(\alpha_{1}, \ldots, \alpha_{n}\right) \in$ $\mathbb{N}^{n}$. For $\alpha$ and $\beta$ multi-indices $\xi \in \mathbb{R}^{n}$ then write

$$
\begin{aligned}
& |\alpha|=\alpha_{1}+\cdots+\alpha_{n}, \quad \partial^{\alpha}=\partial_{x_{1}}^{\alpha_{1}} \cdots \partial_{x_{n}}^{\alpha_{n}}, \quad \xi^{\alpha}=\xi_{1}^{\alpha_{1}} \cdots \xi_{n}^{\alpha_{n}}, \\
& \beta \leq \alpha, \text { if } \beta_{1} \leq \alpha_{1}, \ldots, \beta_{n} \leq \alpha_{n}, \quad\left(\begin{array}{c}
\alpha \\
\beta
\end{array}\right)=\left(\begin{array}{c}
\alpha_{1} \\
\beta_{1}
\end{array}\right) \cdots\left(\begin{array}{c}
\alpha_{n} \\
\beta_{n}
\end{array}\right) \text { if } \beta \leq \alpha .
\end{aligned}
$$

2. The continuous case. Let $\Omega$ be a bounded open subset of $\mathbb{R}^{n}$ with $\mathscr{C}^{2}$ boundary. Let $\omega$ be a nonempty open subset of $\Omega$ such that $\omega \Subset \Omega$. Let $T_{*}>0$ and $Q=\left(0, T_{*}\right) \times \Omega$. We shall use the notation $\nabla=\left(\partial_{t}, \nabla_{x}\right)^{t}$ here and we denote by $n$ the outward unit normal to $Q$ on $\partial Q$ and by $n_{x}$ the outward unit normal to $\Omega$ on $\partial \Omega$. We consider the operator $\mathcal{A}=-\nabla_{x} \cdot\left(\gamma \nabla_{x}\right)$ defined on $\Omega$ with domain $D(\mathcal{A})=H^{2}(\Omega) \cap H_{0}^{1}(\Omega)$ (homogeneous Dirichlet boundary conditions).

The Lebeau-Robbiano spectral inequality of Theorem 1.1 measures the loss of orthogonality of the eigenfunctions $\left(\phi_{k}\right)_{k \in \mathbb{N}^{*}}$, when restricted to $\omega$. It yields the null controllability of the associated parabolic equation through a semi-explicit construction of the control function, which makes use of the natural parabolic exponential decay of the solution (see e.g. [LR95, LZ98a, Mil06, LL09]). Other applications can be found in [JL99].

In this section we give a proof of the Lebeau-Robbiano inequality that differs from the original proof provided in [LR95]. Specifically, the proof in [LR95] relies on an interpolation inequality, itself based on local Carleman estimates. Here, we do not rely on such an interpolation inequality and use a global Carleman estimate instead. The alternative method we propose will be used in the sequel for the discrete version $\mathcal{A}^{\mathfrak{M}}$ of the operator $\mathcal{A}$.

From the regularity of the boundary we may choose a function $\psi$ that satisfies the following property. We enlarge the open set $\Omega$ to a larger open set $\tilde{\Omega}$ as this will be needed for the discrete case in the following sections.

Assumption 2.1. Let $\tilde{\Omega}$ be a smooth open and connected neighborhood of $\bar{\Omega}$ in $\mathbb{R}^{n}$ and set $\tilde{Q}=\left(0, T_{*}\right) \times \tilde{\Omega}$. The function $\psi$ is in $\mathscr{C}^{2}(\tilde{Q}, \mathbb{R})$ and satisfies, for some $c>0$,

$$
\begin{array}{ll}
|\nabla \psi| \geq c \text { and } \psi>0 \text { in } \tilde{Q}, & \partial_{n_{x}} \psi(t, x)<0 \text { in }\left(0, T_{*}\right) \times V_{\partial \Omega}, \\
\partial_{t} \psi \geq c \text { on }\{0\} \times(\Omega \backslash \omega), & \nabla_{x} \psi=0 \text { and } \partial_{t} \psi \leq-c \text { on }\left\{T_{*}\right\} \times \Omega,
\end{array}
$$

where $V_{\partial \Omega}$ is a sufficiently small neighborhood of $\partial \Omega$ in $\tilde{\Omega}$, in which the outward unit normal $n_{x}$ to $\Omega$ is extended from $\partial \Omega$.

Such a function can be obtained by following the technique of [FI96], i.e., making use of Morse functions and the associated approximation theorem [AE84]. Some details of the construction of $\psi$ are given in Appendix C.

With such a function $\psi$, we define the weight function $\varphi:=e^{\lambda \psi}$. We denote by $\varphi\left(T_{*}\right)$ the constant value taken by $\varphi$ over $\left\{T_{*}\right\} \times \bar{\Omega}$. We have the following global Carleman estimate for the elliptic operator $P=-\partial_{t}^{2}+\mathcal{A}$. 
TheOREm 2.2. For $\lambda \geq 1$ sufficiently large, there exist $C>0$ and $s_{0} \geq 1$, both depending on $\Omega, \omega, T_{*}$, and $\operatorname{reg}(\gamma)$, such that

$$
\begin{aligned}
& s^{3}\left\|e^{s \varphi} u\right\|_{L^{2}(Q)}^{2}+s\left\|e^{s \varphi} \nabla u\right\|_{L^{2}(Q)}^{2}+s\left|e^{s \varphi(0, .)} \partial_{t} u(0, .)\right|_{L^{2}(\Omega)}^{2} \\
& +s e^{2 s \varphi\left(T_{*}\right)}\left|\partial_{t} u\left(T_{*}, .\right)\right|_{L^{2}(\Omega)}^{2}+s^{3} e^{2 s \varphi\left(T_{*}\right)}\left|u\left(T_{*}, .\right)\right|_{L^{2}(\Omega)}^{2} \\
& \quad \leq C\left(\left\|e^{s \varphi} P u\right\|_{L^{2}(Q)}^{2}+s e^{2 s \varphi\left(T_{*}\right)}\left|\nabla_{x} u\left(T_{*}, .\right)\right|_{L^{2}(\Omega)}^{2}+s\left|e^{s \varphi(0, .)} \partial_{t} u(0, .)\right|_{L^{2}(\omega)}^{2}\right),
\end{aligned}
$$

for $s \geq s_{0}$, and for all $u \in H^{2}(Q)$, satisfying $\left.u\right|_{\{0\} \times \Omega}=0,\left.u\right|_{\left(0, T_{*}\right) \times \partial \Omega}=0$.

REMARK 2.3. Note that we do not impose any boundary condition for $u$ on $\left\{T_{*}\right\} \times \Omega$. The proof of the Carleman estimate can be found in Appendix 3.A of [Le 07]. Note also that letting the step size $h$ go to zero in the discrete Carleman estimate of Theorem 4.1 below yields a proof for Theorem 2.2.

With this global Carleman estimate we can now prove the Lebeau-Robbiano inequality.

Proof of Theorem 1.1. We set $u(t, x)=\sum_{\mu_{j} \leq \mu} \alpha_{j} \frac{\sinh \left(\sqrt{\mu_{j}} t\right)}{\sqrt{\mu_{j}}} \phi_{j}(x)$. We observe that $u$ satisfies $P u=0,\left.u\right|_{\{0\} \times \Omega}=0$ and $\left.u\right|_{\left(0, T_{*}\right) \times \partial \Omega}=0$. Simply keeping the fifth term in the l.h.s. of (2.1) we have

$$
s^{3} e^{2 s \varphi\left(T_{*}\right)}\left|u\left(T_{*}, .\right)\right|_{L^{2}(\Omega)}^{2} \leq C\left(s e^{2 s \varphi\left(T_{*}\right)}\left|\nabla_{x} u\left(T_{*}, .\right)\right|_{L^{2}(\Omega)}^{2}+s\left|e^{s \varphi(0, .)} \partial_{t} u(0, .)\right|_{L^{2}(\omega)}^{2}\right),
$$

for all $s \geq s_{0}>0$. We note that

$$
\left|u\left(T_{*}, .\right)\right|_{L^{2}(\Omega)}^{2}=\sum_{\mu_{j} \leq \mu}\left|\alpha_{j} \frac{\sinh \left(T_{*} \sqrt{\mu_{j}}\right)}{\sqrt{\mu_{j}}}\right|^{2} \geq T_{*}^{2} \sum_{\mu_{j} \leq \mu}\left|\alpha_{j}\right|^{2},
$$

since the eigenfunctions $\left(\phi_{k}\right)_{k \in \mathbb{N}}$ are chosen orthonormal in $L^{2}$ (recall that the $L^{2}$ inner product is defined in (1.4)). We furthermore note that

$$
\left|e^{s \varphi(0, .)} \partial_{t} u(0, .)\right|_{L^{2}(\omega)} \leq e^{s \sup \varphi(0, .)}\left|\partial_{t} u(0, .)\right|_{L^{2}(\omega)}=e^{s \sup \varphi(0, .)}\left|\sum_{\mu_{j} \leq \mu} \alpha_{j} \phi_{j}(x)\right|_{L^{2}(\omega)},
$$

where the supremum is taken for $x \in \bar{\Omega}$. The result will thus follow if we prove

$$
\frac{1}{2} s^{2}\left|u\left(T_{*}, .\right)\right|_{L^{2}(\Omega)}^{2} \geq C\left|\nabla_{x} u\left(T_{*}, .\right)\right|_{L^{2}(\Omega)}^{2}
$$

for $s \geq C \sqrt{\mu}$. We write

$$
\left|u\left(T_{*}, .\right)\right|_{L^{2}(\Omega)}^{2}=\sum_{\mu_{j} \leq \mu}\left|\alpha_{j} \frac{\sinh \left(T_{*} \sqrt{\mu_{j}}\right)}{\sqrt{\mu_{j}}}\right|^{2} \geq \frac{1}{\mu} \sum_{\mu_{j} \leq \mu}\left|\alpha_{j} \sinh \left(T_{*} \sqrt{\mu_{j}}\right)\right|^{2},
$$

and

$\left|\nabla_{x} u\left(T_{*}, .\right)\right|_{L^{2}(\Omega)}^{2} \leq \frac{1}{\gamma_{\min }}\left(\nabla_{x} u\left(T_{*}, .\right), \gamma \nabla_{x} u\left(T_{*}, .\right)\right)_{L^{2}(\Omega)} \leq \frac{1}{\gamma_{\min }} \sum_{\mu_{j} \leq \mu}\left|\alpha_{j} \sinh \left(T_{*} \sqrt{\mu_{j}}\right)\right|^{2}$,

since the functions $\left(\nabla_{x} \phi_{k}\right)_{k \in \mathbb{N}}$ satisfy $\left(\nabla_{x} \phi_{k}, \gamma \nabla_{x} \phi_{l}\right)_{L^{2}(\Omega)}=\mu_{k} \delta_{k l}, k, l \in \mathbb{N}$. We thus see that condition (2.2) is fulfilled for $s^{2} \geq C \mu$. 
3. Some preliminary discrete calculus results. Here, to prepare for Section 4 , we only consider constant-step discretizations, i.e., $h_{i+\frac{1}{2}}=h, i=0, \ldots, N$.

This section aims to provide calculus rules for discrete operators such as $D, \bar{D}$ and also to provide estimates for the successive applications of such operators on the weight functions. To avoid cumbersome notation we introduce the following continuous difference and averaging operators. For a function $f$ defined on $\mathbb{R}$ we set

$$
\begin{aligned}
& \tau^{+} f(x):=f(x+h / 2), \quad \tau^{-} f(x):=f(x-h / 2), \\
& \text { D } f:=\left(\tau^{+}-\tau^{-}\right) f / h, \quad \hat{f}=\left(\tau^{+}+\tau^{-}\right) f / 2 .
\end{aligned}
$$

REMARK 3.1. To iterate averaging symbols we shall sometimes write $\mathrm{A} f=\hat{f}$, and thus $\mathrm{A}^{2} f=\hat{\hat{f}}$.

Discrete versions of the results we give below will be natural; with the notation given in the introduction, for a function $f$ continuously defined on $\mathbb{R}$, the discrete function $D f$ is in fact $\mathrm{D} f$ sampled on the dual mesh, $\overline{\mathfrak{M}}$, and $\bar{D} f_{d}$ is $\mathrm{D} f$ sampled on the primal mesh, $\mathfrak{M}$. We shall use similar meanings for averaging symbols, $\tilde{f}, \bar{f}$ (see (1.5) and (1.6)), and for more general combinations: for instance $\widetilde{\overline{D D f}}$ will be the function $\widehat{\mathrm{DD} f}$ sampled on $\overline{\mathfrak{M}}$.

3.1. Discrete calculus formulae. We provide calculus results for the finitedifference operators that were defined in the introductory section.

Lemma 3.2. Let the functions $f_{1}$ and $f_{2}$ be continuously defined over $\mathbb{R}$. We have

$$
\mathrm{D}\left(f_{1} f_{2}\right)=\mathrm{D}\left(f_{1}\right) \hat{f}_{2}+\hat{f}_{1} \mathrm{D}\left(f_{2}\right) .
$$

Note that the immediate translation of the proposition to discrete functions $f_{1}, f_{2} \in$ $\mathbb{C}^{\mathfrak{M}}$, and $g_{1}, g_{2} \in \mathbb{C}^{\bar{M}}$ is

$$
D\left(f_{1} f_{2}\right)=D\left(f_{1}\right) \tilde{f}_{2}+\tilde{f}_{1} D\left(f_{2}\right), \quad \bar{D}\left(g_{1} g_{2}\right)=\bar{D}\left(g_{1}\right) \bar{g}_{2}+\bar{g}_{1} \bar{D}\left(g_{2}\right) .
$$

Proof. We have

$$
\begin{aligned}
\mathrm{D}\left(f_{1} f_{2}\right)(x) & =h^{-1}\left(f_{1} f_{2}\right)(x+h / 2)-h^{-1}\left(f_{1} f_{2}\right)(x-h / 2) \\
& =\left(\mathrm{D} f_{1}\right)(x)\left(\tau^{+} f_{2}\right)(x)+\left(\tau^{-} f_{1}\right)(x)\left(\mathrm{D} f_{2}\right)(x) .
\end{aligned}
$$

For symmetry reasons we also have $\mathrm{D}\left(f_{1} f_{2}\right)=\mathrm{D}\left(f_{1}\right) \tau^{-}\left(f_{2}\right)+\tau^{+}\left(f_{1}\right) \mathrm{D}\left(f_{2}\right)$. Averaging the two equations we obtain the result. $\square$

LEMma 3.3. Let the functions $f_{1}$ and $f_{2}$ be continuously defined over $\mathbb{R}$. We then have

$$
\widehat{f_{1} f_{2}}=\hat{f}_{1} \hat{f}_{2}+\frac{h^{2}}{4} \mathrm{D}\left(f_{1}\right) \mathrm{D}\left(f_{2}\right) .
$$

Note that the immediate translation of the proposition to discrete functions $f_{1}, f_{2} \in$ $\mathbb{C}^{\mathfrak{M}}, g_{1}, g_{2} \in \mathbb{C}^{\bar{M}}$ is

$$
\widetilde{f_{1} f_{2}}=\tilde{f}_{1} \tilde{f}_{2}+\frac{h^{2}}{4} D\left(f_{1}\right) D\left(f_{2}\right), \quad \overline{g_{1} g_{2}}=\bar{g}_{1} \bar{g}_{2}+\frac{h^{2}}{4} \bar{D}\left(g_{1}\right) \bar{D}\left(g_{2}\right) .
$$

Some of the following properties can be extended in such a manner to discrete functions. We shall not always write it explicitly. 
Proof. We have

$$
\begin{aligned}
4 \hat{f}_{1} \hat{f}_{2} & =\left(\tau^{+} f_{1}+\tau^{-} f_{1}\right)\left(\tau^{+} f_{2}+\tau^{-} f_{2}\right)=2 \tau^{+}\left(f_{1} f_{2}\right)+2 \tau^{-}\left(f_{1} f_{2}\right) \\
& +\tau^{+} f_{1}\left(\tau^{-} f_{2}-\tau^{+} f_{2}\right)+\tau^{-} f_{1}\left(\tau^{+} f_{2}-\tau^{-} f_{2}\right) \\
& =4 \widehat{f_{1} f_{2}}-h^{2}\left(\mathrm{D} f_{1}\right)\left(D f_{2}\right) . \square
\end{aligned}
$$

Averaging a function twice gives the following formula.

Lemma 3.4. Let the function $f$ be continuously defined over $\mathbb{R}$. We have

$$
\mathrm{A}^{2} f:=\hat{\hat{f}}=f+\frac{h^{2}}{4} \mathrm{DD} f
$$

Proof. We have

$$
\begin{aligned}
4 \hat{\hat{f}} & =\left(\left(\tau^{+}\right)^{2} f+\left(\tau^{-}\right)^{2} f+2 f\right)=4 f+\left(\left(\tau^{+}\right)^{2} f+\left(\tau^{-}\right)^{2} f-2 f\right) \\
& =4 f+h\left(\tau^{+}(\mathrm{D} f)-\tau^{-}(\mathrm{D} f)\right)=4 f+h^{2} \mathrm{DD} f . \mathbf{\square}
\end{aligned}
$$

The following proposition covers discrete integrations by parts and related formulae.

Proposition 3.5. Let $f \in \mathbb{C}^{\mathfrak{M} \cup 2 \mathfrak{M}}$ and $g \in \mathbb{C}^{\bar{M}}$. We have the following formulae:

$$
\begin{aligned}
& \int_{\Omega} f\left(\tau^{+} g\right)=\int_{\Omega}\left(\tau^{-} f\right) g-h f_{0} g_{\frac{1}{2}}, \quad \int_{\Omega} f\left(\tau^{-} g\right)=\int_{\Omega}\left(\tau^{+} f\right) g-h f_{N+1} g_{N+\frac{1}{2}}, \\
& \int_{\Omega} f(\bar{D} g)=-\int_{\Omega}(D f) g+f_{N+1} g_{N+\frac{1}{2}}-f_{0} g_{\frac{1}{2}}, \quad \int_{\Omega} f \bar{g}=\int_{\Omega} \tilde{f} g-\frac{h}{2} f_{N+1} g_{N+\frac{1}{2}}-\frac{h}{2} f_{0} g_{\frac{1}{2}} .
\end{aligned}
$$

Lemma 3.6. Let $f$ be a smooth function on $\mathbb{R}$. We have

$$
\begin{aligned}
& \tau^{ \pm} f=f \pm \frac{h}{2} \int_{0}^{1} \partial_{x} f(. \pm \sigma h / 2) d \sigma, \quad \mathrm{A}^{j} f=f+C_{j} h^{2} \int_{-1}^{1}(1-|\sigma|) \partial_{x}^{2} f\left(.+l_{j} \sigma h\right) d \sigma \\
& \mathrm{D}^{j} f=\partial_{x}^{j} f+C_{j}^{\prime} h^{2} \int_{-1}^{1}(1-|\sigma|)^{j+1} \partial_{x}^{j+2} f\left(.+l_{j} \sigma h\right) d \sigma, \quad j=1,2, \quad l_{1}=\frac{1}{2}, l_{2}=1 .
\end{aligned}
$$

Proof. The results follow from Taylor formulae,

$$
f(x+y)=\sum_{j=0}^{n-1} \frac{y^{j}}{j !} f^{(j)}(x)+y^{n} \int_{0}^{1} \frac{(1-\sigma)^{n-1}}{(n-1) !} f^{(n)}(x+\sigma y) d \sigma,
$$

at order $n=1$ for the first result, order $n=2$ for the second one and orders $n=3$ and 4 for the last one.

3.2. Calculus results related to the weight functions. We now provide some technical lemmata related to discrete operations performed on the Carleman weight functions that is of the form $e^{s \varphi}$ with $\psi \in \mathscr{C}^{k}$, with $k$ sufficiently large. For concision, we set $r=e^{s \varphi}$ and $\rho=r^{-1}$. The positive parameters $s$ and $h$ will be large and small respectively and we are particularly interested in the dependence on $s, h$ and $\lambda$ in the following basic estimates.

We assume $s \geq 1$ and $\lambda \geq 1$. We shall use multi-indices of the form $\alpha=\left(\alpha_{t}, \alpha_{x}\right) \in$ $\mathbb{N}^{2}$. The proofs can be found in Appendix A. 
Lemma 3.7. Let $\alpha$ and $\beta$ be multi-indices. We have

$$
\begin{aligned}
\partial^{\beta}\left(r \partial^{\alpha} \rho\right)= & |\alpha|^{|\beta|}(-s \varphi)^{|\alpha|} \lambda^{|\alpha+\beta|}(\nabla \psi)^{\alpha+\beta} \\
& +|\alpha||\beta|(s \varphi)^{|\alpha|} \lambda^{|\alpha+\beta|-1} \mathcal{O}(1)+s^{|\alpha|-1}|\alpha|(|\alpha|-1) \mathcal{O}_{\lambda}(1)=\mathcal{O}_{\lambda}\left(s^{|\alpha|}\right) .
\end{aligned}
$$

Let $\sigma \in[-1,1]$. We have

$$
\partial^{\beta}\left(r(x)\left(\partial^{\alpha} \rho\right)(x+\sigma h)\right)=\mathcal{O}_{\lambda}\left(s^{|\alpha|}\left(1+(s h)^{|\beta|}\right)\right) e^{\mathcal{O}_{\lambda}(s h)} .
$$

Provided sh $\leq \mathfrak{K}$ we have $\partial^{\beta}\left(r(x)\left(\partial^{\alpha} \rho\right)(x+\sigma h)\right)=\mathcal{O}_{\lambda, \mathfrak{K}}\left(s^{|\alpha|}\right)$. The same expressions hold with $r$ and $\rho$ interchanged and with $s$ changed into $-s$.

With Leibniz formula we have the following estimate.

Corollary 3.8. Let $\alpha, \beta$ and $\delta$ be multi-indices. We have

$$
\begin{aligned}
\partial^{\delta}\left(r^{2}\left(\partial^{\alpha} \rho\right) \partial^{\beta} \rho\right)= & |\alpha+\beta|^{|\delta|}(-s \varphi)^{|\alpha+\beta|} \lambda^{|\alpha+\beta+\delta|}(\nabla \psi)^{\alpha+\beta+\delta} \\
& +|\delta||\alpha+\beta|(s \varphi)^{|\alpha+\beta|} \lambda^{|\alpha+\beta+\delta|-1} \mathcal{O}(1) \\
& +s^{|\alpha+\beta|-1}(|\alpha|(|\alpha|-1)+|\beta|(|\beta|-1)) \mathcal{O}_{\lambda}(1)=\mathcal{O}_{\lambda}\left(s^{|\alpha+\beta|}\right) .
\end{aligned}
$$

Proposition 3.9. Let $\alpha$ be a multi-index. Provided sh $\leq \mathfrak{K}$, we have

$$
\begin{aligned}
r \tau^{ \pm} \partial^{\alpha} \rho & =r \partial^{\alpha} \rho+s^{|\alpha|} \mathcal{O}_{\lambda, \mathfrak{K}}(s h)=s^{|\alpha|} \mathcal{O}_{\lambda, \mathfrak{K}}(1), \\
r \mathrm{~A}^{j} \partial^{\alpha} \rho & =r \partial^{\alpha} \rho+s^{|\alpha|} \mathcal{O}_{\lambda, \mathfrak{K}}\left((s h)^{2}\right)=s^{|\alpha|} \mathcal{O}_{\lambda, \mathfrak{K}}(1), \quad j=1,2, \\
r \mathrm{~A}^{j} \mathrm{D} \rho & =r \partial_{x} \rho+s \mathcal{O}_{\lambda, \mathfrak{K}}\left((s h)^{2}\right)=s \mathcal{O}_{\lambda, \mathfrak{K}}(1), \quad j=0,1 \\
r \mathrm{D}^{2} \rho & =r \partial_{x}^{2} \rho+s^{2} \mathcal{O}_{\lambda, \mathfrak{K}}\left((s h)^{2}\right)=s^{2} \mathcal{O}_{\lambda, \mathfrak{K}}(1) .
\end{aligned}
$$

The same estimates hold with $\rho$ and $r$ interchanged.

Lemma 3.10. Let $\alpha, \beta$ be multi-indices and $k=1,2, j=1,2$. Provided $s h \leq \mathfrak{K}$, we have

$$
\begin{aligned}
& \mathrm{D}^{k}\left(\partial^{\beta}\left(r \partial^{\alpha} \rho\right)\right)=\partial_{x}^{k} \partial^{\beta}\left(r \partial^{\alpha} \rho\right)+h^{2} \mathcal{O}_{\lambda, \mathfrak{K}}\left(s^{|\alpha|}\right), \\
& \mathrm{A}^{j} \partial^{\beta}\left(r \partial^{\alpha} \rho\right)=\partial^{\beta}\left(r \partial^{\alpha} \rho\right)+h^{2} \mathcal{O}_{\lambda, \mathfrak{K}}\left(s^{|\alpha|}\right) .
\end{aligned}
$$

Let $\sigma \in[-1,1]$, we have $\mathrm{D}^{k} \partial^{\beta}\left(r(x) \partial^{\alpha} \rho(x+\sigma h)\right)=\mathcal{O}_{\lambda, \mathfrak{K}}\left(s^{|\alpha|}\right)$. The same expressions hold with $r$ and $\rho$ interchanged.

Lemma 3.11. Let $\alpha, \beta, \delta$ be multi-indices and $k=1,2, j=1,2$. Provided sh $\leq \mathfrak{K}$, we have

$$
\begin{aligned}
& \mathrm{A}^{j} \partial^{\delta}\left(r^{2}\left(\partial^{\alpha} \rho\right) \partial^{\beta} \rho\right)=\partial^{\delta}\left(r^{2}\left(\partial^{\alpha} \rho\right) \partial^{\beta} \rho\right)+h^{2} \mathcal{O}_{\lambda, \mathfrak{K}}\left(s^{|\alpha|+|\beta|}\right)=\mathcal{O}_{\lambda, \mathfrak{K}}\left(s^{|\alpha|+|\beta|}\right), \\
& \mathrm{D}^{k} \partial^{\delta}\left(r^{2}\left(\partial^{\alpha} \rho\right) \partial^{\beta} \rho\right)=\partial_{x}^{k}\left(\partial^{\delta}\left(r^{2}\left(\partial^{\alpha} \rho\right) \partial^{\beta} \rho\right)\right)+h^{2} \mathcal{O}_{\lambda, \mathfrak{K}}\left(s^{|\alpha|+|\beta|}\right)=\mathcal{O}_{\lambda, \mathfrak{K}}\left(s^{|\alpha|+|\beta|}\right) .
\end{aligned}
$$

Let $\sigma, \sigma^{\prime} \in[-1,1]$. We have

$$
\begin{aligned}
& \mathrm{A}^{j} \partial^{\delta}\left(r(x)^{2}\left(\partial^{\alpha} \rho(x+\sigma h)\right) \partial^{\beta} \rho\left(x+\sigma^{\prime} h\right)\right)=\mathcal{O}_{\lambda, \mathfrak{K}}\left(s^{|\alpha|+|\beta|}\right), \\
& \mathrm{D}^{k} \partial^{\delta}\left(r(x)^{2}\left(\partial^{\alpha} \rho(x+\sigma h)\right) \partial^{\beta} \rho\left(x+\sigma^{\prime} h\right)\right)=\mathcal{O}_{\lambda, \mathfrak{K}}\left(s^{|\alpha|+|\beta|}\right) .
\end{aligned}
$$

The same expressions hold with $r$ and $\rho$ interchanged. 
Proposition 3.12.

Let $\alpha$ be a multi-index. For $k=0,1,2, j=0,1,2$, and for sh $\leq \mathfrak{K}$, we have

$\mathrm{D}^{k} \mathrm{~A}^{j} \partial^{\alpha}(r \widehat{\mathrm{D} \rho})=\partial_{x}^{k} \partial^{\alpha}\left(r \partial_{x} \rho\right)+s \mathcal{O}_{\lambda, \mathfrak{K}}\left((s h)^{2}\right)=s \mathcal{O}_{\lambda, \mathfrak{K}}(1)$,

$\mathrm{D}^{k}\left(r \mathrm{D}^{2} \rho\right)=\partial_{x}^{k}\left(r \partial_{x}^{2} \rho\right)+s^{2} \mathcal{O}_{\lambda, \mathfrak{K}}\left((s h)^{2}\right)=s^{2} \mathcal{O}_{\lambda, \mathfrak{K}}(1), \quad \mathrm{D}^{k}\left(r \mathrm{~A}^{2} \rho\right)=\mathcal{O}_{\lambda, \mathfrak{K}}\left((s h)^{2}\right)$.

The same expressions hold with $r$ and $\rho$ interchanged.

Proposition 3.13. Let $\alpha$ and $\beta$ be multi-indices and $k=0,1,2, j=0,1,2$ Provided sh $\leq \mathfrak{K}$ we have

$$
\begin{aligned}
& \mathrm{A}^{j} \mathrm{D}^{k} \partial^{\beta}\left(r^{2}\left(\partial^{\alpha} \rho\right) \widehat{\mathrm{D} \rho}\right)=\partial_{x}^{k} \partial^{\beta}\left(r^{2}\left(\partial^{\alpha} \rho\right) \partial_{x} \rho\right)+s^{|\alpha|+1} \mathcal{O}_{\lambda, \mathfrak{K}}\left((s h)^{2}\right)=s^{|\alpha|+1} \mathcal{O}_{\lambda, \mathfrak{K}}(1), \\
& \mathrm{A}^{j} \mathrm{D}^{k} \partial^{\beta}\left(r^{2}\left(\partial^{\alpha} \rho\right) \mathrm{A}^{2} \rho\right)=\partial_{x}^{k} \partial^{\beta}\left(r\left(\partial^{\alpha} \rho\right)\right)+s^{|\alpha|} \mathcal{O}_{\lambda, \mathfrak{K}}\left((s h)^{2}\right)=s^{|\alpha|} \mathcal{O}_{\lambda, \mathfrak{K}}(1), \\
& \mathrm{A}^{j} \mathrm{D}^{k} \partial^{\beta}\left(r^{2}\left(\partial^{\alpha} \rho\right) \mathrm{D}^{2} \rho\right)=\partial_{x}^{k} \partial^{\beta}\left(r^{2}\left(\partial^{\alpha} \rho\right) \partial_{x}^{2} \rho\right)+s^{|\alpha|+2} \mathcal{O}_{\lambda, \mathfrak{K}}\left((s h)^{2}\right)=s^{|\alpha|+2} \mathcal{O}_{\lambda, \mathfrak{K}}(1), \\
& \mathrm{A}^{j} \mathrm{D}^{k} \partial^{\alpha}\left(r^{2} \widehat{\mathrm{D} \rho} \mathrm{D}^{2} \rho\right)=\partial_{x}^{k} \partial^{\alpha}\left(r^{2}\left(\partial_{x} \rho\right) \partial_{x}^{2} \rho\right)+s^{3} \mathcal{O}_{\lambda, \mathfrak{K}}\left((s h)^{2}\right)=s^{3} \mathcal{O}_{\lambda, \mathfrak{K}}(1), \\
& \mathrm{A}^{j} \mathrm{D}^{k} \partial^{\alpha}\left(r^{2} \widehat{\mathrm{D} \rho} \mathrm{A}^{2} \rho\right)=\partial_{x}^{k} \partial^{\alpha}\left(r \partial_{x} \rho\right)+s \mathcal{O}_{\lambda, \mathfrak{K}}\left((s h)^{2}\right)=s \mathcal{O}_{\lambda, \mathfrak{K}}(1) .
\end{aligned}
$$

REMARK 3.14. We set $\mathrm{D}_{2}:=\left(\left(\tau^{+}\right)^{2}-\left(\tau^{-}\right)^{2}\right) / 2 h=\mathrm{AD}$ and $\mathrm{A}_{2}:=\left(\left(\tau^{+}\right)^{2}+\right.$ $\left.\left(\tau^{-}\right)^{2}\right) / 2$. We see that the results in the previous Lemmata and Propositions are preserved when we replace some of the $D$ by $D_{2}$ and some of the $A$ by $A_{2}$.

4. A semi-discrete elliptic Carleman estimate for uniform meshes. Here we consider constant-step discretizations. The case of non-uniform meshes is treated in the following section.

For any uniform mesh $\mathfrak{M}$, let $\xi_{1} \in \mathbb{R}^{\mathfrak{M}}$ and $\xi_{2} \in \mathbb{R}^{\bar{M}}$ be two positive discrete functions. We denote by $\operatorname{reg}(\xi)$ the following quantity

$$
\operatorname{reg}(\xi)=\max \left(\sup _{\mathfrak{M}}\left(\xi_{1}+\frac{1}{\xi_{1}}\right), \sup _{\overline{\mathfrak{M}}}\left(\xi_{2}+\frac{1}{\xi_{2}}\right), \sup _{\overline{\mathfrak{M}}}\left|D \xi_{1}\right|, \sup _{\mathfrak{M}}\left|\bar{D} \xi_{2}\right|\right) .
$$

Hence, $\operatorname{reg}(\xi)$ measures the boundedness of $\xi_{1}$ and $\xi_{2}$ and of their discrete derivatives as well as the distance to zero of $\xi_{1}$ and $\xi_{2}$.

We extend $\xi_{1}$ and $\xi_{2}$ to piecewise affine functions in the neighborhood $\tilde{\Omega}$ of $\Omega$ on the dual and the primal meshes respectively. Continuous versions of the previous properties are then satisfied. We also call $\xi_{1}$ and $\xi_{2}$ the two piecewise affine functions. Note that $\xi_{2, d}$ gives the discrete function $\xi_{2}$ we started from.

We let $\omega \Subset \Omega$ be a nonempty open subset. We set the operator $P^{\mathfrak{m}}$ to be $P^{\mathfrak{M}}=-\left(\xi_{1} \partial_{t}^{2}+\bar{D}\left(\xi_{2, d} D\right)\right)$, continuous in the variable $t \in\left(0, T_{*}\right)$, with $T_{*}>0$, and discrete in the variable $x \in \Omega$.

The Carleman weight function is of the form $r=e^{s \varphi}$ with $\varphi=e^{\lambda \psi}$, where $\psi \in \mathscr{C}^{k}(\overline{\tilde{Q}})$, with $k \in \mathbb{N}$ sufficiently large, satisfies Assumption 2.1. Here, to treat the semi-discrete case, we shall use the enlarged neighborhood $\tilde{\Omega}$ of $\Omega$ introduced in Assumption 2.1. This will allow multiple actions of discrete operators such as D and A on the weight functions. In particular we take $\psi$ such that $\partial_{x} \psi \geq 0$ in $\left(0, T_{*}\right) \times V_{a}$ and $\partial_{x} \psi \geq 0$ in $\left(0, T_{*}\right) \times V_{b}$ where $V_{a}$ and $V_{b}$ are neighborhoods of $a$ and $b$ respectively. This then yields

$$
(r \overline{D \rho})_{0} \leq 0, \quad(r \overline{D \rho})_{N+1} \geq 0
$$


We recall that $\rho=r^{-1}$. We introduce the following notation

$$
\nabla_{\xi} f=\left(\xi_{1}^{\frac{1}{2}} \partial_{t} f, \xi_{2}^{\frac{1}{2}} \partial_{x} f\right)^{t}, \quad \Delta_{\xi} f=\xi_{1} \partial_{t}^{2} f+\xi_{2} \partial_{x}^{2} f .
$$

We prove the following semi-discrete Carleman estimate. The function $u$ denotes a function that is continuously defined and regular $\left(\mathscr{C}^{2}\right)$ w.r.t. $t$ and discrete w.r.t. $x$.

TheOrem 4.1. Let $\mathrm{reg}^{0}>0$ be given. For the parameter $\lambda \geq 1$ sufficiently large, there exist $C, s_{0} \geq 1, h_{0}>0, \varepsilon_{0}>0$, depending on $\omega, T_{*}, \mathrm{reg}^{0}$, such that for any $\xi=\left(\xi_{1}, \xi_{2}\right)$ with $\operatorname{reg}(\xi) \leq \operatorname{reg}^{0}$, we have

$$
\begin{gathered}
s^{3}\left\|e^{s \varphi} u\right\|_{L^{2}(Q)}^{2}+s\left\|e^{s \varphi} \partial_{t} u\right\|_{L^{2}(Q)}^{2}+s\left\|e^{s \varphi_{d}} D u\right\|_{L^{2}(Q)}^{2}+s\left|e^{s \varphi(0, .)} \partial_{t} u(0, .)\right|_{L^{2}(\Omega)}^{2} \\
+s e^{2 s \varphi\left(T_{*}\right)}\left|\partial_{t} u\left(T_{*}, .\right)\right|_{L^{2}(\Omega)}^{2}+s^{3} e^{2 s \varphi\left(T_{*}\right)}\left|u\left(T_{*}, .\right)\right|_{L^{2}(\Omega)}^{2} \\
\leq C\left(\left\|e^{s \varphi} P^{M} u\right\|_{L^{2}(Q)}^{2}+s e^{2 s \varphi\left(T_{*}\right)}\left|D u\left(T_{*}, .\right)\right|_{L^{2}(\Omega)}^{2}+s\left|e^{s \varphi(0, .)} \partial_{t} u(0, .)\right|_{L^{2}(\omega)}^{2}\right),
\end{gathered}
$$

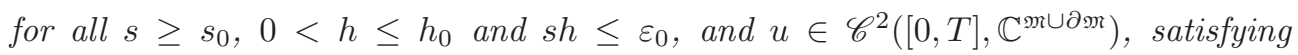
$\left.u\right|_{\{0\} \times \Omega}=0,\left.u\right|_{\left(0, T_{*}\right) \times \partial \Omega}=0$.

The proof of some of the lemmata below can be found in Appendix B.

Proof. We set $f:=-P^{\mathfrak{m}} u$. At first, we shall work with the function $v=r u$, i.e., $u=\rho v$, that satisfies

$$
r\left(\xi_{1} \partial_{t}^{2}(\rho v)+\bar{D}\left(\xi_{2, d} D(\rho v)\right)\right)=r f .
$$

By Lemma 3.2, we have $\partial_{t}^{2}(\rho v)=\left(\partial_{t}^{2} \rho\right) v+2\left(\partial_{t} \rho\right) \partial_{t} v+\rho \partial_{t}^{2} v$ and

$$
\bar{D}\left(\xi_{2, d} D(\rho v)\right)=\left(\bar{D}\left(\xi_{2, d} D \rho\right)\right) \overline{\tilde{v}}+\overline{\xi_{2, d} D \rho} \bar{D} \tilde{v}+(\bar{D} \tilde{\rho}) \overline{\xi_{2, d} D v}+\overline{\tilde{\rho}} \bar{D}\left(\xi_{2, d} D v\right),
$$

since $r \rho=1$. By Lemma 3.3 we have

$\overline{\xi_{2, d} D v}=\overline{\xi_{2, d}} \overline{D v}+\frac{h}{4}\left(\bar{D} \xi_{2, d}\right)\left(\tau^{+} D v-\tau^{-} D v\right), \quad \overline{\xi_{2, d} D \rho}=\overline{\xi_{2, d}} \overline{D \rho}+\frac{h^{2}}{4}\left(\bar{D} \xi_{2, d}\right)(\bar{D} D \rho)$, $\bar{D}\left(\xi_{2, d} D \rho\right)=\left(\bar{D} \xi_{2, d}\right) \overline{D \rho}+\overline{\xi_{2, d}} \bar{D} D \rho$.

Equation (4.3) thus reads $A v+B_{1} v=g^{\prime}$ where

$$
\begin{aligned}
A v= & \underbrace{\xi_{1} \partial_{t}^{2} v+r \overline{\tilde{\rho}} \bar{D}\left(\xi_{2, d} D v\right)}_{A_{1} v}+\underbrace{\xi_{1} r\left(\partial_{t}^{2} \rho\right) v+\xi_{2} r(\bar{D} D \rho) \overline{\tilde{v}}}_{A_{2} v}, \\
B_{1} v= & 2 \xi_{1} r\left(\partial_{t} \rho\right) \partial_{t} v+2 r \overline{D \rho} \xi_{2} \overline{D v}, \\
g^{\prime}= & r f-\frac{h}{4} r \overline{D \rho}\left(\bar{D} \xi_{2, d}\right)\left(\tau^{+} D v-\tau^{-} D v\right)-\frac{h^{2}}{4}\left(\bar{D} \xi_{2, d}\right) r(\bar{D} D \rho) \overline{D v} \\
& -h \mathcal{O}(1) r \overline{D \rho} \overline{D v}-\left(r\left(\bar{D} \xi_{2, d}\right) \overline{D \rho}+h \mathcal{O}(1) r(\bar{D} D \rho)\right) \overline{\tilde{v}},
\end{aligned}
$$

since $\bar{D} \tilde{w}=\overline{D w}$, for any function $w$ and since $\left\|\overline{\xi_{2, d}}-\xi_{2}\right\|_{\infty} \leq C h$. Following [FI96] we now set

$$
B v=\underbrace{2 \xi_{1} r\left(\partial_{t} \rho\right) \partial_{t} v+2 r \overline{D \rho} \xi_{2} \overline{D v}}_{B_{1} v} \underbrace{-2 s\left(\Delta_{\xi} \varphi\right) v}_{B_{2} v}, \quad g=g^{\prime}-2 s\left(\Delta_{\xi} \varphi\right) v .
$$


Equation (4.3) now reads $A v+B v=g$ and we write

$$
\|A v\|_{L^{2}(Q)}^{2}+\|B v\|_{L^{2}(Q)}^{2}+2 \operatorname{Re}(A v, B v)_{L^{2}(Q)}=\|g\|_{L^{2}(Q)}^{2} .
$$

We shall need the following estimation of $\|g\|_{L^{2}(Q)}$.

Lemma 4.2 (Estimate of the r.h.s.). For $s h \leq \mathfrak{K}$ we have

$$
\|g\|_{L^{2}(Q)}^{2} \leq C_{\lambda, \mathfrak{K}}\left(\|r f\|_{L^{2}(Q)}^{2}+s^{2}\|v\|_{L^{2}(Q)}^{2}+(s h)^{2}\|D v\|_{L^{2}(Q)}^{2}\right) .
$$

Most of the remaining of the proof will be dedicated to computing the innerproduct $\operatorname{Re}(A v, B v)_{L^{2}(Q)}$. Developing the inner-product $\operatorname{Re}(A v, B v)_{L^{2}(Q)}$, we set $I_{i j}=\operatorname{Re}\left(A_{i} v, B_{j} v\right)_{L^{2}(Q)}$.

Note that all the estimates depend on $\mathrm{reg}^{0}$, which is a bound of the regularity measure $\operatorname{reg}(\xi)$ of $\xi_{1}$ and $\xi_{2}$.

LEMma 4.3 (Estimate of $I_{11}$ ). For $s h \leq \mathfrak{K}$ we have $I_{11} \geq I_{11}^{a}+W_{11}+Y_{11}-X_{11}-$ $J_{11}$, with

$$
\begin{aligned}
I_{11}^{a}= & -s \lambda^{2} \iint_{Q} \xi_{1} \varphi\left|\nabla_{\xi} \psi\right|^{2}\left|\partial_{t} v\right|^{2} d t-s \lambda^{2} \iint_{Q}\left(\xi_{2} \varphi\left|\nabla_{\xi} \psi\right|^{2}\right)_{d}|D v|^{2} d t \\
& -s \lambda\left[\int_{\Omega} \xi_{1}^{2} \varphi\left(\partial_{t} \psi\right)\left|\partial_{t} v\right|^{2}\right]_{0}^{T_{*}}+s \lambda \int_{\Omega}\left(\xi_{1} \xi_{2} \varphi \partial_{t} \psi\right)_{d}\left(T_{*}\right)|D v|^{2}\left(T_{*}\right),
\end{aligned}
$$

and

$$
\begin{aligned}
Y_{11} & =\int_{0}^{T_{*}}\left(1+\mathcal{O}_{\lambda, \mathfrak{K}}\left((s h)^{2}\right)\right)\left(\left(\xi_{2} \overline{\xi_{2, d}} r \overline{D \rho}\right)_{N+1}|D v|_{N+\frac{1}{2}}^{2}-\left(\xi_{2} \overline{\xi_{2, d}} r \overline{D \rho}\right)_{0}|D v|_{\frac{1}{2}}^{2}\right) d t, \\
X_{11} & =\iint_{Q} \beta_{11}\left|\partial_{t} v\right|^{2} d t+\iint_{Q} \nu_{11}|D v|^{2} d t+\operatorname{Re} \iint_{Q} \alpha_{11}^{(1)} \widetilde{\partial_{t} v} D v^{*} d t \\
J_{11} & =\int_{\Omega} \delta_{11}|D v|^{2}\left(T_{*}\right), \quad W_{11}=\iint_{Q} \gamma_{11}\left|D \partial_{t} v\right|^{2} d t
\end{aligned}
$$

where

$$
\begin{aligned}
& \gamma_{11}=\frac{1}{2} h^{2} s \lambda^{2}\left(\xi_{1} \varphi\left|\nabla_{\xi} \psi\right|^{2}\right)_{d}+h^{2} s \lambda \varphi_{d} \mathcal{O}(1)+h \mathcal{O}_{\lambda, \mathfrak{K}}\left((s h)^{2}\right), \quad \delta_{11}=s \mathcal{O}_{\lambda, \mathfrak{K}}(s h), \\
& \beta_{11}=s \lambda \varphi \mathcal{O}(1)+s \mathcal{O}_{\lambda, \mathfrak{K}}(s h)+h \mathcal{O}_{\lambda}(1), \quad \nu_{11}=s \lambda \varphi_{d} \mathcal{O}(1)+s \mathcal{O}_{\lambda, \mathfrak{K}}(s h), \\
& \alpha_{11}^{(1)}=s \lambda \varphi_{d} \mathcal{O}(1)+s \mathcal{O}_{\lambda, \mathfrak{K}}(s h) .
\end{aligned}
$$

Lemma 4.4 (Estimate of $I_{12}$ ). For $s h \leq \mathfrak{K}$, the term $I_{12}$ is of the following form

$$
I_{12}=2 s \lambda^{2} \iint_{Q} \xi_{1} \varphi\left|\nabla_{\xi} \psi\right|^{2}\left|\partial_{t} v\right|^{2} d t+2 s \lambda^{2} \iint_{Q}\left(\varphi \xi_{2}\left|\nabla_{\xi} \psi\right|^{2}\right)_{d}|D v|^{2} d t-X_{12}-J_{12},
$$

with

$$
\begin{aligned}
X_{12} & =\iint_{Q} \beta_{12}\left|\partial_{t} v\right|^{2} d t+\iint_{Q} \nu_{12}|D v|^{2} d t+\iint_{Q} \mu_{12}|v|^{2} d t+\operatorname{Re} \iint_{Q} \alpha_{12}^{(1)} \tilde{v}^{*} D v d t, \\
J_{12} & =\operatorname{Re} \int_{\Omega}\left(\alpha_{12}^{(2)} v^{*} \partial_{t} v\right)\left(T_{*}\right)+\int_{\Omega} \eta_{12}|v|^{2}\left(T_{*}\right),
\end{aligned}
$$


where

$$
\begin{aligned}
\beta_{12} & =s \lambda \varphi \mathcal{O}(1), \quad \nu_{12}=s \lambda \varphi \mathcal{O}(1)+s \mathcal{O}_{\lambda, \mathfrak{K}}\left(h+(s h)^{2}\right), \quad \mu_{12}=s \mathcal{O}_{\lambda, \mathfrak{K}}(1), \\
\alpha_{12}^{(1)} & =s \mathcal{O}_{\lambda, \mathfrak{K}}(1), \quad \alpha_{12}^{(2)}=s \mathcal{O}_{\lambda, \mathfrak{K}}(1) \quad \text { and } \eta_{12}=s \mathcal{O}_{\lambda}(1) .
\end{aligned}
$$

LEMMA 4.5 (Estimate of $I_{21}$ ). For $s h \leq \mathfrak{K}$, the term $I_{21}$ can be estimated as

$$
\begin{aligned}
I_{21} \geq & 3 s^{3} \lambda^{4} \iint_{Q} \varphi^{3}\left|\nabla_{\xi} \psi\right|^{4}|v|^{2} d t-(s \lambda)^{3} \int_{\Omega} \xi_{1}\left(\varphi^{3}\left(\partial_{t} \psi\right)\left|\nabla_{\xi} \psi\right|^{2}\right)\left(T_{*}\right)|v|^{2}\left(T_{*}\right) \\
& +Y_{21}+W_{21}-X_{21}-J_{21}
\end{aligned}
$$

with

$$
\begin{aligned}
W_{21} & =\iint_{Q} \gamma_{21}\left|D \partial_{t} v\right|^{2} d t, \\
Y_{21} & =\int_{0}^{T_{*}} \mathcal{O}_{\lambda, \mathfrak{K}}\left((s h)^{2}\right)(r \overline{D \rho})_{0}|D v|_{\frac{1}{2}}^{2} d t+\int_{0}^{T_{*}} \mathcal{O}_{\lambda, \mathfrak{K}}\left((s h)^{2}\right)(r \overline{D \rho})_{N+1}|D v|_{N+\frac{1}{2}}^{2} d t, \\
X_{21} & =\iint_{Q} \mu_{21}|v|^{2} d t+\iint_{Q} \nu_{21}|D v|^{2} d t, \quad J_{21}=\int_{\Omega} \eta_{21}|v|^{2}\left(T_{*}\right)+\int_{\Omega} \delta_{21}|D v|^{2}\left(T_{*}\right),
\end{aligned}
$$

where

$$
\begin{aligned}
\gamma_{21} & =h \mathcal{O}(s h), \quad \mu_{21}=(s \lambda \varphi)^{3} \mathcal{O}(1)+s^{2} \mathcal{O}_{\lambda}(1)+s^{3} \mathcal{O}_{\lambda, \mathfrak{K}}\left((s h)^{2}\right), \\
\nu_{21} & =s \mathcal{O}_{\lambda, \mathfrak{K}}\left((s h)^{2}\right), \quad \eta_{21}=s^{3} \mathcal{O}_{\lambda, \mathfrak{K}}\left((s h)^{2}\right)+s^{2} \mathcal{O}_{\lambda, \mathfrak{K}}(1), \quad \text { and } \quad \delta_{21}=s \mathcal{O}_{\lambda, \mathfrak{K}}\left((s h)^{2}\right) .
\end{aligned}
$$

LEMMA 4.6 (Estimate of $I_{22}$ ). For sh $\leq \mathfrak{K}$, the term $I_{22}$ is of the following form

$$
I_{22}=-2 s^{3} \lambda^{4} \iint_{Q} \varphi^{3}\left|\nabla_{\xi} \psi\right|^{4}|v|^{2} d t-X_{22}, \quad X_{22}=\iint_{Q} \mu_{22}|v|^{2} d t+\iint_{Q} \nu_{22}|D v|^{2} d t
$$

where $\mu_{22}=(s \lambda \varphi)^{3} \mathcal{O}(1)+s^{2} \mathcal{O}_{\lambda, \mathfrak{K}}(1)+s^{3} \mathcal{O}_{\lambda, \mathfrak{K}}\left((s h)^{2}\right)$ and $\nu_{22}=s \mathcal{O}_{\lambda, \mathfrak{K}}\left((s h)^{2}\right)$.

Continuation of the proof of Theorem 4.1. Collecting the terms we have obtained in the previous lemmata, from (4.4) we obtain, for $s h \leq \mathfrak{K}$,

$$
\begin{gathered}
2 s \lambda^{2} \iint_{Q} \xi_{1} \varphi\left|\nabla_{\xi} \psi\right|^{2}\left|\partial_{t} v\right|^{2} d t+2 s \lambda^{2} \iint_{Q}\left(\varphi \xi_{2}\left|\nabla_{\xi} \psi\right|^{2}\right)_{d}|D v|^{2} d t+2 s^{3} \lambda^{4} \iint_{Q} \varphi^{3}\left|\nabla_{\xi} \psi\right|^{4}|v|^{2} d t \\
-2 s \lambda\left[\int_{\Omega} \xi_{1}^{2} \varphi\left(\partial_{t} \psi\right)\left|\partial_{t} v\right|^{2}\right]_{0}^{T_{*}}+2 s \lambda \int_{\Omega}\left(\xi_{1} \xi_{2} \varphi \partial_{t} \psi\right)_{d}\left(T_{*}\right)|D v|^{2}\left(T_{*}\right) \\
-2(s \lambda)^{3} \int_{\Omega} \xi_{1}\left(\varphi^{3}\left(\partial_{t} \psi\right)\left|\nabla_{\xi} \psi\right|^{2}\right)\left(T_{*}\right)|v|^{2}\left(T_{*}\right)+W+Y \leq C_{\lambda, \mathfrak{K}}\|r f\|_{L^{2}(Q)}^{2}+X+J,
\end{gathered}
$$

where $X=X_{11}+X_{12}+X_{21}+X_{22}+C_{\lambda, \mathfrak{K}} s^{2}\|v\|_{L^{2}(Q)}^{2}+C_{\lambda, \mathfrak{K}}(s h)^{2}\|D v\|_{L^{2}(Q)}^{2}, J=$ $J_{11}+J_{12}+J_{21}, W=W_{11}+W_{21}$ and $Y=Y_{11}+Y_{21}$. With the following lemma, we may in fact ignore the terms $W$ and $Y$.

LEMmA 4.7. Let sh $\leq \mathfrak{K}$. There exists $\lambda_{1} \geq 1$, and $\varepsilon_{1}(\lambda)>0$ such that for $\lambda \geq \lambda_{1}$ and $0<$ sh $\leq \varepsilon_{1}(\lambda)$, we have $W \geq 0$ and $Y \geq 0$. 
LEMMA 4.8. We have

$$
\begin{aligned}
& \left|\operatorname{Re} \iint_{Q} \alpha_{11}^{(1)} \widetilde{\partial_{t} v} D v^{*} d t\right| \leq \iint_{Q} \beta_{11}^{(2)}\left|\partial_{t} v\right|^{2} d t+\iint_{Q} \nu_{11}^{(2)}|D v|^{2} d t, \\
& \left|\operatorname{Re} \iint_{Q} \alpha_{12}^{(1)} \tilde{v}^{*} D v d t\right| \leq C s^{2} \iint_{Q} \mathcal{O}_{\lambda, \mathfrak{K}}(1)|v|^{2} d t+C \iint_{Q}|D v|^{2} d t, \\
& \left|\operatorname{Re} \int_{\Omega}\left(\alpha_{12}^{(2)} v^{*} \partial_{t} v\right)\left(T_{*}\right)\right| \leq C \int_{\Omega}\left|\partial_{t} v\right|^{2}\left(T_{*}\right)+s^{2} \int_{\Omega} \mathcal{O}_{\lambda, \mathfrak{K}}(1)|v|^{2}\left(T_{*}\right),
\end{aligned}
$$

with

$$
\beta_{11}^{(2)}=s \lambda \varphi \mathcal{O}(1)+s \mathcal{O}_{\lambda, \mathfrak{K}}(s h), \quad \nu_{11}^{(2)}=s \lambda \varphi_{d} \mathcal{O}(1)+s \mathcal{O}_{\lambda, \mathfrak{K}}(s h)
$$

Recalling the properties satisfied by $\psi$ listed in Assumption 2.1, if we choose $\lambda_{2} \geq \lambda_{1}$ sufficiently large, then for $\lambda=\lambda_{2}$ (fixed for the rest of the proof) and $s h \leq \varepsilon_{1}\left(\lambda_{2}\right)$, from (4.6) and Lemmata 4.7 and 4.8, we obtain

$$
\begin{aligned}
& s^{3}\|v\|_{L^{2}(Q)}^{2}+s\left\|\partial_{t} v\right\|_{L^{2}(Q)}^{2}+s\|D v\|_{L^{2}(Q)}^{2} \\
& +s\left|\partial_{t} v(0, .)\right|_{L^{2}(\Omega)}^{2}+s\left|\partial_{t} v\left(T_{*}, .\right)\right|_{L^{2}(\Omega)}^{2}+s^{3}\left|v\left(T_{*}, .\right)\right|_{L^{2}(\Omega)}^{2} \\
& \quad \leq C_{\lambda_{2}, \mathfrak{K}}\left(\|r f\|_{L^{2}(Q)}^{2}+s\left|D v\left(T_{*}, .\right)\right|_{L^{2}(\Omega)}^{2}+s\left|\partial_{t} v(0, .)\right|_{L^{2}(\omega)}^{2}\right)+\tilde{X}+\tilde{J},
\end{aligned}
$$

where

$$
\begin{aligned}
& \tilde{X}=\iint_{Q} \mu_{1}|v|^{2} d t+\iint_{Q} \beta_{1}\left|\partial_{t} v\right|^{2} d t+\iint_{Q} \nu_{1}|D v|^{2} d t \\
& \tilde{J}=\int_{\Omega} \eta_{1}|v|^{2}\left(T_{*}\right)+\int_{\Omega} \alpha_{1}\left|\partial_{t} v\right|^{2}\left(T_{*}\right)+\int_{\Omega} \delta_{1}|D v|^{2}\left(T_{*}\right),
\end{aligned}
$$

with

$$
\begin{aligned}
\mu_{1} & =s^{2} \mathcal{O}_{\lambda_{2}, \mathfrak{K}}(1)+s^{3} \mathcal{O}_{\lambda_{2}, \mathfrak{K}}\left((s h)^{2}\right), \quad \beta_{1}=s \mathcal{O}_{\lambda_{2}, \mathfrak{K}}(s h), \\
\nu_{1} & =s \mathcal{O}_{\lambda_{2}, \mathfrak{K}}(h+s h)+\mathcal{O}_{\lambda_{2}, \mathfrak{K}}(1), \quad \eta_{1}=s^{2} \mathcal{O}_{\lambda_{2}, \mathfrak{K}}(1)+s^{3} \mathcal{O}_{\lambda_{2}, \mathfrak{K}}\left((s h)^{2}\right), \\
\alpha_{1} & =s \mathcal{O}_{\lambda_{2}, \mathfrak{K}}\left((s h)^{2}\right)+C, \quad \delta_{1}=s \mathcal{O}_{\lambda_{2}, \mathfrak{K}, \eta}(1) .
\end{aligned}
$$

We can now choose $\varepsilon_{0}$ and $h_{0}$ sufficiently small, with $0<\varepsilon_{0} \leq \varepsilon_{1}\left(\lambda_{2}\right)$, and $s_{0} \geq 1$ sufficiently large, such that for $s \geq s_{0}, 0<h \leq h_{0}$, and $s h \leq \varepsilon_{0}$, we obtain

$$
\begin{aligned}
& s^{3}\|v\|_{L^{2}(Q)}^{2}+s\left\|\partial_{t} v\right\|_{L^{2}(Q)}^{2}+s\|D v\|_{L^{2}(Q)}^{2} \\
& +s\left|\partial_{t} v(0, .)\right|_{L^{2}(\Omega)}^{2}+s\left|\partial_{t} v\left(T_{*}, .\right)\right|_{L^{2}(\Omega)}^{2}+s^{3}\left|v\left(T_{*}, .\right)\right|_{L^{2}(\Omega)}^{2} \\
& \quad \leq C_{\lambda_{2}, \mathfrak{K}, \varepsilon_{0}, s_{0}}\left(\|r f\|_{L^{2}(Q)}^{2}+s\left|D v\left(T_{*}, .\right)\right|_{L^{2}(\Omega)}^{2}+s\left|\partial_{t} v(0, .)\right|_{L^{2}(\omega)}^{2}\right) .
\end{aligned}
$$

We now proceed with using back the unknown function $u$ in the estimates. In fact we have the following lemma.

LEMma 4.9. For $s h \leq \mathfrak{K}$ we have

$$
\begin{aligned}
& \left\|r_{d} D u\right\|_{L^{2}(Q)}^{2} \leq C_{\lambda, \mathfrak{K}}\left(s^{2}\|v\|_{L^{2}(Q)}^{2}+\|D v\|_{L^{2}(Q)}^{2}\right) \\
& \left\|r \partial_{t} u\right\|_{L^{2}(Q)}^{2} \leq C_{\lambda, \mathfrak{K}} s^{2}\left(\|v\|_{L^{2}(Q)}^{2}+\left\|\partial_{t} v\right\|_{L^{2}(Q)}^{2}\right) \\
& \left|r \partial_{t} u\left(T_{*}, .\right)\right|_{L^{2}(\Omega)}^{2} \leq C_{\lambda, \mathfrak{K}}\left(s^{2}\left|v\left(T_{*}, .\right)\right|_{L^{2}(\Omega)}^{2}+\left|\partial_{t} v\left(T_{*}, .\right)\right|_{L^{2}(\Omega)}^{2}\right) .
\end{aligned}
$$


Since $\varphi\left(T_{*}\right)=$ Cst by the properties of $\psi$ (see Assumption 2.1) and because of the zero-boundary condition imposed on $u$ at $t=0$ we have

$$
\begin{aligned}
& \left|\partial_{t} v(0, .)\right|_{L^{2}(\Omega)}^{2}=\left|r \partial_{t} u(0, .)\right|_{L^{2}(\Omega)}^{2}, \quad\left|\partial_{t} v(0, .)\right|_{L^{2}(\omega)}^{2}=\left|r \partial_{t} u(0, .)\right|_{L^{2}(\omega)}^{2}, \\
& \left|D v\left(T_{*}, .\right)\right|_{L^{2}(\Omega)}^{2}=r\left(T_{*}\right)^{2}\left|D u\left(T_{*}, .\right)\right|_{L^{2}(\Omega)}^{2} .
\end{aligned}
$$

We hence obtained the desired Carleman estimate from (4.8) and Lemma 4.9.

REMARK 4.10. Note that the term $W$ in (4.6), that we proved to be non-negative, has no counterpart in the continuous case.

5. Carleman estimates for regular non uniform meshes. We present in this section a way to extend the above results to the class of non uniform meshes introduced in Section 1.2, see also Figure 1.1. We chose a function $\vartheta$ satisfying (1.7) to remain fixed in the sequel.

By using first-order Taylor formulae we obtain the following result.

Lemma 5.1. Let us define $\zeta \in \mathbb{R}^{\bar{M}}$ and $\bar{\zeta} \in \mathbb{R}^{\mathfrak{M}}$ as follows

$$
\zeta_{i+\frac{1}{2}}=\frac{h_{i+\frac{1}{2}}}{h^{\star}}, \forall i \in\{0, \ldots, N\}, \quad \bar{\zeta}_{i}=\frac{h_{i}}{h^{\star}}, \forall i \in\{1, \ldots, N\} .
$$

These two discrete functions are connected to the geometry of the primal and dual meshes $\mathfrak{M}$ and $\overline{\mathfrak{M}}$, and we have

$$
\begin{gathered}
0<\inf _{\Omega_{0}} \vartheta^{\prime} \leq \zeta_{i+\frac{1}{2}} \leq \sup _{\Omega_{0}} \vartheta^{\prime}, \forall i \in\{0, \ldots, N\}, \\
0<\inf _{\Omega_{0}} \vartheta^{\prime} \leq \bar{\zeta}_{i} \leq \sup _{\Omega_{0}} \vartheta^{\prime}, \quad \forall i \in\{1, \ldots, N\} . \\
|\bar{D} \zeta|_{L^{\infty}(\Omega)} \leq \frac{\left\|\vartheta^{\prime \prime}\right\|_{L^{\infty}}}{\inf _{\Omega_{0}} \vartheta^{\prime}}, \quad|D \bar{\zeta}|_{L^{\infty}(\Omega)} \leq \frac{\left\|\vartheta^{\prime \prime}\right\|_{L^{\infty}}}{\inf _{\Omega_{0}} \vartheta^{\prime}} .
\end{gathered}
$$

We aim to prove uniform Carleman estimates in this framework by using the result on uniform meshes of Section 4 . To any $u \in \mathbb{C}^{\mathfrak{M} \cup \partial \mathfrak{m}}$, we associate the discrete function denoted by $\mathcal{Q}_{\mathfrak{M}}^{\mathfrak{M}_{0}} u \in \mathbb{C}^{\mathfrak{M}_{0}} \cup \mathfrak{M}_{0}$ defined on the uniform mesh $\mathfrak{M}_{0}$ which takes the same values as $u$ at the corresponding nodes. More precisely, if $u=$ $\sum_{i=1}^{N} \mathbf{1}_{\left[x_{i-\frac{1}{2}}, x_{i+\frac{1}{2}}\right]} u_{i}$, we let $\mathcal{Q}_{\mathfrak{M}}^{\mathfrak{M}_{0}} u=\sum_{i=1}^{N} \mathbf{1}_{\left[\left(i-\frac{1}{2}\right) h^{\star},\left(i+\frac{1}{2}\right) h^{\star}\right]} u_{i}$, and $\left(\mathcal{Q}_{\mathfrak{M}}^{\mathfrak{M}_{0}} u\right)_{0}=u_{0}$, $\left(\mathcal{Q}_{\mathfrak{M}}^{\mathfrak{M}_{0}} u\right)_{N+1}=u_{N+1}$. Similarly, for any $u \in \mathbb{C}^{\overline{\mathfrak{M}}}, u=\sum_{i=0}^{N} \mathbf{1}_{\left[x_{i}, x_{i+1}\right]} u_{i+\frac{1}{2}}$, we set $\mathcal{Q}_{\bar{M}}^{\overline{M_{0}}} u=\sum_{i=0}^{N} \mathbf{1}_{\left[i h^{\star},(i+1) h^{\star}\right]} u_{i+\frac{1}{2}}$. The operators $\mathcal{Q}_{\mathfrak{M}}^{\mathfrak{M}_{0}}$ and $\mathcal{Q}_{\bar{M}}^{\bar{M} 0}$ are invertible and we denote by $\mathcal{Q}_{\mathfrak{M}_{0}}^{\mathfrak{M}}$ and $\mathcal{Q} \overline{\mathfrak{M}_{0}}$ their respective inverses. Let us now give commutation properties between these operators and discrete difference operators. To lighten notation we shall use the same symbols $D$ (resp. $\bar{D}$ ) for the difference operators acting on $\mathbb{C}^{\mathfrak{M}_{0} \cup \partial \mathfrak{M}_{0}}$ and $\mathbb{C}^{\mathfrak{M} \cup \partial \mathfrak{M}}$ (resp. on $\mathbb{C}^{\overline{\mathfrak{M}_{0}}}$ and $\mathbb{C}^{\overline{\mathfrak{M}}}$ ).

LEMMA 5.2.

1. For any $u \in \mathbb{C}^{\mathfrak{M} \cup \partial \mathfrak{M}}$ and any $v \in \mathbb{C}^{\overline{\mathfrak{M}}}$, we have

$$
D\left(\mathcal{Q}_{\mathfrak{M}}^{\mathfrak{M}_{0}} u\right)=\mathcal{Q}_{\overline{\mathfrak{M}}}^{\overline{\mathfrak{M}_{0}}}(\zeta D u), \quad \bar{D}\left(\mathcal{Q}_{\overline{\mathfrak{M}}}^{\overline{\mathfrak{M}_{0}}} v\right)=\mathcal{Q}_{\mathfrak{M}}^{\mathfrak{M}_{0}}(\bar{\zeta} \bar{D} v) .
$$


2. For any $u \in \mathbb{C}^{\mathfrak{M} \cup \partial \mathfrak{M}}$ we have

$$
\bar{D}\left(\gamma_{d} D u\right)=(\bar{\zeta})^{-1} \mathcal{Q}_{\mathfrak{M}_{0}}^{\mathfrak{M}}\left(\bar{D}\left(\left(\mathcal{Q}_{\overline{\mathfrak{M}}}^{\overline{\mathfrak{M}_{0}}} \frac{\gamma_{d}}{\zeta}\right) D\left(\mathcal{Q}_{\mathfrak{M}}^{\mathfrak{M}_{0}} u\right)\right)\right)
$$

Proof. Let $0 \leq i \leq N$. On the one hand, by the definitions of $\mathcal{Q}_{\mathfrak{M}}^{\mathfrak{M}_{0}}$ and $D$ acting on $\mathbb{C}^{\mathfrak{M}_{0} \cup \partial \mathfrak{M}_{0}}$, we have

$$
\left(D\left(\mathcal{Q}_{\mathfrak{M}}^{\mathfrak{M}_{0}} u\right)\right)_{i+\frac{1}{2}}=\frac{\left(\mathcal{Q}_{\mathfrak{M}}^{\mathfrak{M}_{0}} u\right)_{i+1}-\left(\mathcal{Q}_{\mathfrak{M}}^{\mathfrak{M}_{0}} u\right)_{i}}{h^{\star}}=\frac{u_{i+1}-u_{i}}{h^{\star}} .
$$

On the other hand, by the definitions of $\zeta, \mathcal{Q}_{\overline{\mathfrak{M}}}^{\overline{\mathfrak{M}}}$, and $D$ acting on $\mathbb{C}^{\mathfrak{M} \cup \partial \mathfrak{M}}$ we have

$$
\left(\mathcal{Q}_{\overline{\mathfrak{M}}}^{\overline{\mathcal{M}_{0}}}(\zeta D u)\right)_{i+\frac{1}{2}}=(\zeta D u)_{i+\frac{1}{2}}=\frac{h_{i+\frac{1}{2}}}{h^{\star}} \frac{u_{i+1}-u_{i}}{h_{i+\frac{1}{2}}}=\frac{u_{i+1}-u_{i}}{h^{\star}},
$$

which proves the first result. The other statements can be proven in a similar manner.

口

LEMma 5.3. For any $u \in \mathbb{C}^{\mathfrak{M}}$ and any $v \in \mathbb{C}^{\overline{\mathfrak{M}}}$ we have

$$
\begin{gathered}
\left(\sup _{\Omega_{0}} \vartheta^{\prime}\right)^{-1}|u|_{L^{2}(\Omega)}^{2} \leq\left|\mathcal{Q}_{\mathfrak{M}_{0}}^{\mathfrak{M}_{0}} u\right|_{L^{2}\left(\Omega_{0}\right)}^{2} \leq\left(\inf _{\Omega_{0}} \vartheta^{\prime}\right)^{-1}|u|_{L^{2}(\Omega)}^{2}, \\
\left(\sup _{\Omega_{0}} \vartheta^{\prime}\right)^{-1}|v|_{L^{2}(\Omega)}^{2} \leq\left|\mathcal{Q}_{\overline{\mathfrak{M}}}^{\overline{\mathfrak{M}_{0}}} v\right|_{L^{2}\left(\Omega_{0}\right)}^{2} \leq\left(\inf _{\Omega_{0}} \vartheta^{\prime}\right)^{-1}|v|_{L^{2}(\Omega)}^{2} .
\end{gathered}
$$

Furthermore, the same inequalities hold by replacing $\Omega$ by $\omega$ and $\Omega_{0}$ by $\omega_{0}$, respectively.

Proof. By definition of $\mathcal{Q}_{\mathfrak{M}}^{\mathfrak{M}_{0}}$ and of the discrete norms, we have

$$
\left|\mathcal{Q}_{\mathfrak{M}}^{\mathfrak{M}_{0}} u\right|_{L^{2}\left(\Omega_{0}\right)}^{2}=\sum_{i=1}^{N} h^{\star}\left|u_{i}\right|^{2}=\sum_{i=1}^{N} \frac{1}{\bar{\zeta}_{i}} h_{i}\left|u_{i}\right|^{2} \text {, and }|u|_{L^{2}(\Omega)}^{2}=\sum_{i=1}^{N} h_{i}\left|u_{i}\right|^{2},
$$

so that the first property follows from Lemma 5.1. The property for $v$ is proved similarly.

To avoid any ambiguity we introduce the following notation. For any continuous function $f$ defined on $\bar{\Omega}$ (resp. on $\overline{\Omega_{0}}$ ) we denote by $\Pi_{\mathfrak{M}} f=\left(f\left(x_{i}\right)\right)_{0 \leq i \leq N+1} \in \mathbb{C}^{\mathfrak{M} \cup \partial \mathfrak{M}}$ the sampling of $f$ on $\mathfrak{M}$ (resp. $\Pi_{\mathfrak{M}_{0}} f=\left(f\left(i h^{\star}\right)\right)_{0 \leq i \leq N+1} \in \mathbb{C}^{\mathfrak{M}_{0} \cup \partial \mathfrak{M}_{0}}$ the sampling of $f$ on $\left.\mathfrak{M}_{0}\right)$.

LEMMA 5.4. Let $f$ be a continuous function defined on $\Omega$. We have

$$
\mathcal{Q}_{\mathfrak{M}}^{\mathfrak{M}_{0}} \Pi_{\mathfrak{M}} f=\Pi_{\mathfrak{M}_{0}}(f \circ \vartheta)
$$

In particular, for any $u \in \mathbb{C}^{\mathfrak{M} \cup \partial \mathfrak{M}}$ we have

$$
\mathcal{Q}_{\mathfrak{M}}^{\mathfrak{M}_{0}}\left(\left(\Pi_{\mathfrak{M}} f\right) u\right)=\Pi_{\mathfrak{M}_{0}}(f \circ \vartheta)\left(\mathcal{Q}_{\mathfrak{M}}^{\mathfrak{M}_{0}} u\right)
$$

We can now prove the following discrete Carleman estimate for our elliptic operator $P^{\mathfrak{M}}=-\partial_{t}^{2}-\bar{D}\left(\gamma_{d} D \cdot\right)$ on the mesh $\mathfrak{M}$.

THEOREM 5.5. Let $\vartheta$ satisfy (1.7) and $\psi$ be a weight function satisfying assumption 2.1 for the observation domain $\omega$. For the parameter $\lambda \geq 1$ sufficiently large, 
there exist $C, s_{0} \geq 1, h_{0}>0, \varepsilon_{0}>0$, depending on $\omega, T_{*}, \vartheta$, $\operatorname{reg}(\gamma)$, such that for any mesh $\mathfrak{M}$ obtained from $\vartheta$ by (1.8), we have

$$
\begin{gathered}
s^{3}\left\|e^{s \varphi} u\right\|_{L^{2}(Q)}^{2}+s\left\|e^{s \varphi} \partial_{t} u\right\|_{L^{2}(Q)}^{2}+s\left\|e^{s \varphi_{d}} D u\right\|_{L^{2}(Q)}^{2}+s\left|e^{s \varphi(0, .)} \partial_{t} u(0, .)\right|_{L^{2}(\Omega)}^{2} \\
+s e^{2 s \varphi\left(T_{*}\right)}\left|\partial_{t} u\left(T_{*}, .\right)\right|_{L^{2}(\Omega)}^{2}+s^{3} e^{2 s \varphi\left(T_{*}\right)}\left|u\left(T_{*}, .\right)\right|_{L^{2}(\Omega)}^{2} \\
\leq C\left(\left\|e^{s \varphi} P^{\mathfrak{M}} u\right\|_{L^{2}(Q)}^{2}+s e^{2 s \varphi\left(T_{*}\right)}\left|D u\left(T_{*}, .\right)\right|_{L^{2}(\Omega)}^{2}+s\left|e^{s \varphi(0, .)} \partial_{t} u(0, .)\right|_{L^{2}(\omega)}^{2}\right),
\end{gathered}
$$

for all $s \geq s_{0}, 0<h \leq h_{0}$ and $s h \leq \varepsilon_{0}$, and $u \in \mathscr{C}^{2}\left([0, T], \mathbb{C}^{\mathfrak{M} \cup \partial \mathfrak{M}}\right)$, satisfying $\left.u\right|_{\{0\} \times \Omega}=0,\left.u\right|_{\left(0, T_{*}\right) \times \partial \Omega}=0$.

Proof. We set $w=\mathcal{Q}_{\mathfrak{M}}^{\mathfrak{M}_{0}} u$ defined on the uniform mesh $\mathfrak{M}_{0}$. By using Lemma 5.2, we have

$$
\mathcal{Q}_{\mathfrak{M}}^{\mathfrak{M}_{0}}\left(\bar{\zeta} P^{\mathfrak{M}} u\right)=\left(\mathcal{Q}_{\mathfrak{M}}^{\mathfrak{M}_{0}} \bar{\zeta}\right) \partial_{t}^{2} w-\bar{D}\left(\left(\mathcal{Q}_{\overline{\mathfrak{M}}}^{\overline{\mathfrak{M}_{0}}} \frac{\gamma_{d}}{\zeta}\right) D w\right)
$$

We see that the right-hand side of (5.2) is a semi-discrete elliptic operator of the form $P^{\mathfrak{M}_{0}}=\xi_{1} \partial_{t}^{2}-\bar{D}\left(\xi_{2} D \cdot\right)$ applied to $w$, where

$$
\xi_{1}=\mathcal{Q}_{\mathfrak{M}}^{\mathfrak{M}_{0}} \bar{\zeta}, \quad \xi_{2}=\mathcal{Q}_{\overline{\mathfrak{M}}}^{\overline{\mathfrak{M}_{0}}} \frac{\gamma_{d}}{\zeta}
$$

By using assumption 2.1 and (1.7), we now observe that , the function $\psi \circ \vartheta:(t, x) \mapsto$ $\psi(t, \vartheta(x))$ is a suitable weight function associated to the control domain $\omega_{0}=\vartheta^{-1}(\omega)$ in $\Omega_{0}$, i.e. that $\psi \circ \vartheta$ satisfies assumption 2.1 for the domains $\Omega_{0}$ and $\omega_{0}$.

In Theorem 4.1, we have obtained a discrete uniform Carleman estimate for $P^{\mathfrak{M}_{0}}$ and the weight function $\psi \circ \vartheta$ on the uniform mesh $\mathfrak{M}_{0}$. We can now deduce the same result on the non-uniform mesh $\mathfrak{M}$.

Firstly, we observe that there exists $C_{\vartheta, \gamma}$ such that we have $\operatorname{reg}(\xi) \leq C_{\vartheta, \gamma}$ uniformly with respect to $h^{\star}$, with $\xi=\left(\xi_{1}, \xi_{2}\right)$ as defined in (5.3). Then, choosing $\operatorname{reg}^{0}=C_{\vartheta, \gamma}$ in the proof of Theorem 4.1, we see that estimate (4.8) holds

$$
\begin{aligned}
& s^{3}\left\|e^{s \varphi \circ \vartheta} w\right\|_{L^{2}\left(Q_{0}\right)}^{2}+s\left\|\partial_{t}\left(e^{s \varphi \circ \vartheta} w\right)\right\|_{L^{2}\left(Q_{0}\right)}^{2}+s\left\|D\left(e^{s(\varphi \circ \vartheta)} w\right)\right\|_{L^{2}\left(Q_{0}\right)}^{2} \\
& +s\left|e^{s \varphi \circ \vartheta(0, .)} \partial_{t} w(0, .)\right|_{L^{2}\left(\Omega_{0}\right)}^{2}+s\left|\partial_{t}\left(e^{2 s \varphi} w\right)\left(T_{*}, .\right)\right|_{L^{2}\left(\Omega_{0}\right)}^{2}+s^{3} e^{2 s \varphi\left(T_{*}\right)}\left|w\left(T_{*}, .\right)\right|_{L^{2}\left(\Omega_{0}\right)}^{2} \\
\leq & C\left(\left\|e^{s \varphi \circ \vartheta} P^{\mathfrak{M}_{0}} w\right\|_{L^{2}\left(Q_{0}\right)}^{2}+s e^{2 s \varphi\left(T_{*}\right)}\left|D w\left(T_{*}, .\right)\right|_{L^{2}\left(\Omega_{0}\right)}^{2}+s\left|e^{s \varphi \circ \vartheta(0, .)} \partial_{t} w(0, .)\right|_{L^{2}\left(\omega_{0}\right)}^{2}\right),
\end{aligned}
$$

and the constant $C$ is uniform in $h^{\star}$ for $s$ sufficiently large and with $s h^{\star} \leq \varepsilon_{0}$, for $\varepsilon_{0}$ sufficiently small. Note that, setting $\tilde{\varepsilon}_{0}=\left(\inf _{\Omega_{0}} \vartheta^{\prime}\right) \varepsilon_{0}$, we see that the condition $s h \leq \tilde{\varepsilon}_{0}$ on the size of the non-uniform mesh $\mathfrak{M}$ implies the condition $s h^{\star} \leq \varepsilon_{0}$ for the uniform mesh $\mathfrak{M}_{0}$.

Secondly, by using the previous lemmata 5.1, 5.2, 5.3, 5.4 and considering each term above separately, we see that we have the following estimates.

- For the third term in the 1.h.s. of (5.4)

$$
\begin{aligned}
&\left\|D\left(e^{s(\varphi \circ \vartheta)} w\right)\right\|_{L^{2}\left(Q_{0}\right)}^{2}=\left\|D\left(e^{s(\varphi \circ \vartheta)} \mathcal{Q}_{\mathfrak{M}}^{\mathfrak{M}_{0}} u\right)\right\|_{L^{2}\left(Q_{0}\right)}^{2}=\left\|D \mathcal{Q}_{\mathfrak{M}}^{\mathfrak{M}_{0}}\left(e^{s \varphi} u\right)\right\|_{L^{2}\left(Q_{0}\right)}^{2} \\
&=\left\|\mathcal{Q}_{\overline{\mathfrak{M}}}^{\overline{\mathcal{M}_{0}}}\left(\zeta D\left(e^{s \varphi} u\right)\right)\right\|_{L^{2}\left(Q_{0}\right)}^{2} \geq\left(\sup _{\Omega_{0}} \vartheta^{\prime}\right)^{-1}\left\|\zeta D\left(e^{s \varphi} u\right)\right\|_{L^{2}(Q)}^{2} \\
& \geq\left(\sup _{\Omega_{0}} \vartheta^{\prime}\right)^{-1}\left(\inf _{\Omega_{0}} \vartheta^{\prime}\right)^{2}\left\|D\left(e^{s \varphi} u\right)\right\|_{L^{2}(Q)}^{2}
\end{aligned}
$$


- For any $\alpha \in\{0,1\}$, we have

$$
\left\|\partial_{t}^{\alpha}\left(e^{s \varphi \circ \vartheta} w\right)\right\|_{L^{2}\left(Q_{0}\right)}^{2}=\left\|\mathcal{Q}_{\mathfrak{M}}^{\mathfrak{m}_{0}} \partial_{t}^{\alpha}\left(e^{s \varphi} u\right)\right\|_{L^{2}\left(Q_{0}\right)}^{2} \geq\left(\sup _{\Omega_{0}} \vartheta^{\prime}\right)^{-1}\left\|\partial_{t}^{\alpha}\left(e^{s \varphi} u\right)\right\|_{L^{2}(Q)}^{2},
$$

and similar inequalities hold for the other terms in the l.h.s. of (5.4).

- By using (5.2) and (5.3) we have

$$
\begin{gathered}
\left.\left\|e^{s \varphi \circ \vartheta} P^{\mathfrak{M}_{0}} w\right\|_{L^{2}\left(Q_{0}\right)}^{2}=\| e^{s \varphi \vartheta \vartheta} \mathcal{Q}_{\mathfrak{M}}^{\mathfrak{M}_{0}}\left(\bar{\zeta} P^{\mathfrak{M}} u\right)\right)\left\|_{L^{2}\left(Q_{0}\right)}^{2}=\right\| \mathcal{Q}_{\mathfrak{M}}^{\mathfrak{M}_{0}}\left(e^{s \varphi} \bar{\zeta} P^{\mathfrak{M}} u\right) \|_{L^{2}\left(Q_{0}\right)}^{2} \\
\leq\left(\inf _{\Omega_{0}} \vartheta^{\prime}\right)^{-1}\left\|e^{s \varphi} \bar{\zeta} P^{\mathfrak{M}} u\right\|_{L^{2}(Q)}^{2} \leq\left(\sup _{\Omega_{0}} \vartheta^{\prime}\right)^{2}\left(\inf _{\Omega_{0}} \vartheta^{\prime}\right)^{-1}\left\|e^{s \varphi} P^{\mathfrak{M}} u\right\|_{L^{2}(Q)}^{2} .
\end{gathered}
$$

- Finally, since $\vartheta\left(\omega_{0}\right)=\omega$, we have

$$
\begin{aligned}
\left|e^{s \varphi \circ \vartheta(0, \cdot)} \partial_{t} w(0, .)\right|_{L^{2}\left(\omega_{0}\right)}^{2}=\left|\mathcal{Q}_{\mathfrak{M}}^{\mathfrak{M}_{0}}\left(e^{s \varphi(0, \cdot)} \partial_{t} u(0, \cdot)\right)\right|_{L^{2}\left(\omega_{0}\right)}^{2} & \\
& \leq\left(\inf _{\Omega_{0}} \vartheta^{\prime}\right)^{-1}\left|e^{s \varphi(0, \cdot)} \partial_{t} u(0, \cdot)\right|_{L^{2}(\omega)}^{2} .
\end{aligned}
$$

The proof is complete.

6. A partial discrete Lebeau-Robbiano spectral inequality. In this section, with the Carleman estimate we just proved, we obtain a Lebeau-Robbiano type spectral inequality for the lower part of the spectrum of the operator $\mathcal{A}^{\mathfrak{M}}$. The constant we shall obtain in this inequality is in fact uniform w.r.t. to the step size of the chosen mesh $\mathfrak{M}$.

We recall that we denote by $\phi^{\mathfrak{m}}$ a set of discrete orthonormal eigenfunctions, $\phi_{j} \in \mathbb{C}^{\mathfrak{M}}, 1 \leq j \leq|\mathfrak{M}|$, of the operator $\mathcal{A}^{\mathfrak{M}}$ with homogeneous Dirichlet boundary conditions, and by $\mu^{\mathfrak{m}}$ the set of the associated eigenvalues sorted in a non-decreasing sequence, $\mu_{j}, 1 \leq j \leq|\mathfrak{M}|$.

TheOREM 6.1 (Partial discrete Lebeau-Robbiano inequality). Let $\vartheta$ satisfying (1.7). There exist $C>0, \varepsilon_{1}>0$ and $h_{0}$ such that, for any mesh $\mathfrak{M}$ obtained from $\vartheta$ by (1.8) such that $h \leq h_{0}$, for all $0<\mu \leq \varepsilon_{1} / h^{2}$, we have

$$
\sum_{\substack{\mu_{k} \in \mu \mathfrak{M} \\ \mu_{k} \leq \mu}}\left|\alpha_{k}\right|^{2}=\int_{\Omega}\left|\sum_{\substack{\mu_{k} \in \mu \mathfrak{M} \\ \mu_{k} \leq \mu}} \alpha_{k} \phi_{k}\right|^{2} \leq C e^{C \sqrt{\mu}} \int_{\omega}\left|\sum_{\substack{\mu_{k} \in \mu \mathfrak{M} \\ \mu_{k} \leq \mu}} \alpha_{k} \phi_{k}\right|^{2}, \quad \forall\left(\alpha_{k}\right)_{1 \leq k \leq|\mathfrak{M}|} \subset \mathbb{C} .
$$

Proof. We adapt the proof presented in Section 2. We introduce the following semi-discrete function $u(t)=\sum_{\mu_{k} \in \mu^{\mathfrak{M}}} \alpha_{k} \frac{\sinh \left(\sqrt{\mu_{k}} t\right)}{\sqrt{\mu_{k}}} \phi_{k}$, which satisfies the boundary conditions listed in the discrete Carleman estimate of Theorem 5.5 and $P^{\mathfrak{M}} u=-\partial_{t}^{2} u+$ $\mathcal{A}^{\mathfrak{M}} u=0$. For some $K>0, s_{0}>0, h_{0}>0$ and $\varepsilon_{0}>0$, uniform w.r.t. $\mathfrak{M}$, we thus have

$$
s^{3} e^{2 s \varphi\left(T_{*}\right)}\left|u\left(T_{*}, .\right)\right|_{L^{2}(\Omega)}^{2} \leq K\left(s e^{2 s \varphi_{d}\left(T_{*}\right)}\left|D u\left(T_{*}, .\right)\right|_{L^{2}(\Omega)}^{2}+s\left|e^{s \varphi(0, .)} \partial_{t} u(0, .)\right|_{L^{2}(\omega)}^{2}\right),
$$

for $s \geq s_{0}, 0<h \leq h_{0}$ and $s h \leq \varepsilon_{0}$. As in the proof of Theorem 1.1 it suffices to obtain $\frac{1}{2} s^{2}\left|u\left(T_{*}\right)\right|_{L^{2}(\Omega)}^{2} \geq K\left|D u\left(T_{*}\right)\right|_{L^{2}(\Omega)}^{2}$. In fact we have

$$
\begin{aligned}
\left|u\left(T_{*}\right)\right|_{L^{2}(\Omega)}^{2} \geq \frac{1}{\mu} \sum_{\mu_{k} \leq \mu}\left|\alpha_{k} \sinh \left(T_{*} \sqrt{\mu_{k}}\right)\right|^{2}, \\
\left|D u\left(T_{*}\right)\right|_{L^{2}(\Omega)}^{2} \leq \frac{1}{\gamma_{\min }} \sum_{\mu_{k} \leq \mu}\left|\alpha_{k} \sinh \left(T_{*} \sqrt{\mu_{k}}\right)\right|^{2},
\end{aligned}
$$


since the discrete functions $D \phi_{k}, 1 \leq j \leq|\mathfrak{M}|$, satisfy $\int_{\Omega} \gamma_{d} D \phi_{k} D \phi_{k}=\delta_{j k} \mu_{k}$. It thus suffices to have $s^{2} /(2 \mu) \geq K / \gamma_{\min }$. Since $s h \leq \varepsilon_{0}$, this can be made possible if $\mu \leq \gamma_{\min } \varepsilon_{0}^{2} /\left(2 K h^{2}\right)$. The result follows with $\varepsilon_{1}=\bar{\gamma}_{\min } \varepsilon_{0}^{2} / 2 K$.

7. Uniform controllability of the lower part of the spectrum. Proof of Theorem 1.4. Let $\vartheta$ satisfy (1.7) and $\mathfrak{M}$ be a mesh defined by (1.8) such that $h \leq h_{0}$. We set $\mu_{\max }^{\mathfrak{m}}=\varepsilon_{1} / h^{2}$, with $h_{0}$ and $\varepsilon_{1}$ given by Theorem 6.1 . Let $j^{\mathfrak{M}}=$ $\max \left\{j ; 2^{2 j} \leq \mu_{\max }^{\mathfrak{M}}\right\}$. We recall the following notation from the introduction

$$
E_{j}=\operatorname{Span}\left\{\phi_{k} ; \mu_{k} \leq 2^{2 j}\right\} \subset \mathbb{C}^{\mathfrak{M}}, \quad j \in \mathbb{N}
$$

and denote by $\Pi_{E_{j}}$ the $L^{2}(\Omega)$-orthogonal projection onto $E_{j}$.

LEMmA 7.1. There exists $C \geq 0$ such that, for $j \leq j^{\mathfrak{M}}$ and $S>0$, the semidiscrete solution $q$ in $\mathscr{C}^{\infty}\left([0, S], E_{j}\right)$ to the adjoint parabolic system

$$
\begin{cases}-\partial_{t} q+\mathcal{A}^{\mathfrak{M}} q=0 & \text { in }(0, S) \times \Omega, \\ q=0 & \text { on }(0, S) \times \partial \Omega \\ q(S)=q_{F} \in E_{j}, & \end{cases}
$$

satisfies the following observability estimate

$$
|q(0)|_{L^{2}(\Omega)}^{2} \leq \frac{C e^{C 2^{j}}}{S} \int_{0}^{S} \int_{\omega}|q(t)|^{2} d t .
$$

Proof. If $q(0)=\sum_{\mu_{k} \leq 2^{2 j}} b_{k} \phi_{k}$. Then $q(t)=\sum_{\mu_{k} \leq 2^{2 j}} \alpha_{k}(t) \phi_{k}$ with $\alpha_{k}(t)=$ $b_{k} e^{\mu_{k} t}$. Parabolic dissipation and Theorem 6.1, since $2^{2 j} \leq \varepsilon_{1} / h^{2}$, then yield

$$
\begin{aligned}
S|q(0)|_{L^{2}(\Omega)}^{2} & \leq \int_{0}^{S}|q(t)|_{L^{2}(\Omega)}^{2} d t=\int_{0}^{S}\left|\sum_{\mu_{k} \leq 2^{2 j}} \alpha_{k}(t) \phi_{k}\right|_{L^{2}(\Omega)}^{2} d t \\
& \leq C e^{C 2^{j}} \int_{0}^{S} \int_{\omega}\left|\sum_{\mu_{k} \leq 2^{2 j}} \alpha_{k}(t) \phi_{k}\right|^{2} d t=C e^{C 2^{j}} \int_{0}^{S} \int_{\omega}|q(t)|^{2} d t .
\end{aligned}
$$

We now consider the following partial control problem

$$
\begin{cases}\partial_{t} y+\mathcal{A}^{\mathfrak{M}} y=\Pi_{E_{j}}\left(\mathbf{1}_{\omega} v\right) & \text { in }(0, S) \times \Omega, \\ y=0 & \text { on }(0, S) \times \partial \Omega, \\ y(0)=y_{0} \in E_{j} & \text { in } \Omega .\end{cases}
$$

With the previous observability result we have the following lemma.

LEMMA 7.2. There exists $C>0$, such that for $j \leq j^{\mathfrak{M}}$, there exists a control function $w \in L^{2}((0, S) \times \Omega)$ that brings the solution to system $(7.2)$ to zero at time $S$, and which satisfies

$$
\|w\|_{L^{2}((0, S) \times \Omega)} \leq C S^{-\frac{1}{2}} e^{C 2^{j}}\left|y_{0}\right|_{L^{2}(\Omega)} .
$$

We shall denote by $V_{j}\left(y_{0}, a, S\right)$ such a control when working on the time interval $(a, a+S)$ instead.

We now present the iterative construction of the control function. We write $[0, T / 2]=\bigcup_{j \in \mathbb{N}}\left[a_{j}, a_{j+1}\right]$, with $a_{0}=0, a_{j+1}=a_{j}+2 T_{j}$, for $j \in \mathbb{N}$ and $T_{j}=K 2^{-j \rho}$ 
with $\rho \in(0,1)$ and the constant $K$ is such that $2 \sum_{j=0}^{\infty} T_{j}=T / 2$. The control function is defined as follows, for $0 \leq j \leq j^{\mathfrak{M}}$,

$$
\text { if } \begin{aligned}
t \in\left(a_{j}, a_{j}+T_{j}\right], \quad v(t)= & V_{j}\left(\Pi_{E_{j}} y\left(a_{j}\right), a_{j}, T_{j}\right) \\
& \text { and } y(t)=S\left(t-a_{j}\right) y\left(a_{j}\right)+\int_{a_{j}}^{t} S(t-s) v(s) d s,
\end{aligned}
$$

if $t \in\left(a_{j}+T_{j}, a_{j+1}\right], \quad v(t)=0$ and $\quad y(t)=S\left(t-a_{j}-T_{j}\right) y\left(a_{j}+T_{j}\right)$,

and $v(t)=0$ for $t \in\left[a_{j \mathfrak{M}}, T\right]$ where $S(t)$ denote the semi-group $S(t)=e^{-t \mathcal{A}^{\mathfrak{M}}}$. In particular, $\|S(t)\|_{\left(L^{2}, L^{2}\right)} \leq 1$. This choice of the control function $v$ in the time interval $\left[a_{j}, a_{j}+T_{j}\right], j \leq j^{\mathfrak{m}}$, implies

$$
\left|y\left(a_{j}+T_{j}\right)\right|_{L^{2}(\Omega)} \leq\left(1+C e^{C 2^{j}}\right)\left|y\left(a_{j}\right)\right|_{L^{2}(\Omega)}, \quad \text { and } \quad \Pi_{E_{j}} y\left(a_{j}+T_{j}\right)=0 .
$$

During the passive period, $t \in\left[a_{j}+T_{j}, a_{j+1}\right]$, there is an exponential decrease of the $L^{2}$ norm, $\left|y\left(a_{j+1}\right)\right|_{L^{2}(\Omega)} \leq e^{-2^{2 j} T_{j}}\left|y\left(a_{j}+T_{j}\right)\right|_{L^{2}(\Omega)}$, and from the value of $T_{k}$ introduced above we thus obtain

$$
\left|y\left(a_{j+1}\right)\right|_{L^{2}(\Omega)} \leq e^{C 2^{j}-K 2^{j(2-\rho)}}\left|y\left(a_{j}\right)\right|_{L^{2}(\Omega)},
$$

which gives $\left|y\left(a_{j+1}\right)\right|_{L^{2}(\Omega)} \leq e^{\sum_{k=0}^{j}\left(C 2^{k}-K 2^{k(2-\rho)}\right)}\left|y_{0}\right|_{L^{2}(\Omega)}$. With $\rho \in(0,1)$, there exists $C>0$, such that

$$
\left|y\left(a_{j+1}\right)\right|_{L^{2}(\Omega)} \leq C e^{-C 2^{j(2-\rho)}}\left|y_{0}\right|_{L^{2}(\Omega)}, \quad 0 \leq j \leq j^{\mathfrak{M}} .
$$

Since $2^{2\left(j^{\mathfrak{M}}+1\right)} \geq \varepsilon_{1} / h^{2}=\mu_{\max }^{\mathfrak{M}}$ it furthermore follows that

$$
\left\|y\left(a_{j \mathfrak{M}}+1\right)\right\|_{L^{2}(\Omega)} \leq C e^{-C / h^{(2-\rho)}}\left\|y_{0}\right\|_{L^{2}(\Omega)} .
$$

The constant $C$ depends only on the map $\vartheta$ defining the mesh $\mathfrak{M}$ but not on the mesh size $h$.

Concerning the $L^{2}$ norm of $v$ we have $\|v\|_{L^{2}(Q)}^{2}=\sum_{0 \leq j \leq j^{\mathfrak{m}}}\|v\|_{L^{2}\left(\left(a_{j}, a_{j}+T_{j}\right) \times \Omega\right)}^{2}$. From Lemma 7.2 and Estimate (7.3) we deduce

$$
\|v\|_{L^{2}((0, T) \times \Omega)}^{2} \leq\left(C T_{0}^{-1} e^{2 C}+\sum_{1 \leq j \leq j^{\mathfrak{M}}} C T_{j}^{-1} e^{C 2^{j}} e^{-C 2^{(j-1)(2-\rho)}}\right)\left|y_{0}\right|_{L^{2}(\Omega)}^{2} .
$$

Hence, arguing as above there exists $0<C_{T}<\infty$, independent of $h$, depending only on $\vartheta$, such that

$$
\|v\|_{L^{2}((0, T) \times \Omega)} \leq C_{T}\left|y_{0}\right|_{L^{2}(\Omega)} .
$$

Since $v(t)=0$ for $t \in\left[a_{j \mathfrak{M}}+T_{j \mathfrak{M}}, T\right]$ and since $2^{2\left(j^{\mathfrak{M}}+1\right)} \geq \varepsilon_{1} / h^{2}=\mu_{\max }^{\mathfrak{M}}$ it furthermore follows that

$$
|y(T)|_{L^{2}(\Omega)} \leq C e^{-(C / h)^{2}}\left|y_{0}\right|_{L^{2}(\Omega)},
$$

as $\Pi_{E_{j} \mathfrak{M}} y\left(a_{j \mathfrak{M}}+T_{j^{\mathfrak{M}}}\right)=0$. This concludes the proof of Theorem 1.4.

REMARK 7.3. If we chose to directly control in the space $E_{j m}$ based on the partial observability result of Lemma 7.1, instead of the Lebeau-Robbiano construction of the 
control function we have done here, we would obtain a $L^{2}$ norm of the control that diverges to $+\infty$ as $h$ goes to zero. The Lebeau-Robbiano construction, making use of the natural parabolic exponential decay, is a key point to obtain a uniform bound for the $L^{2}$ norm of the control.

With the null controllability result we have obtained in $E_{j m}$ in Theorem 1.4, we have the following observability result which improves upon Lemma 7.1.

Corollary 7.4. For $j \leq j^{\mathfrak{M}}$ and $S>0$, the semi-discrete solution $q$ in $\mathscr{C}^{\infty}\left([0, S], E_{j}\right)$ to system (7.1) satisfies the following uniform observability estimate

$$
|q(0)|_{L^{2}(\Omega)} \leq C_{T}\left(\int_{0}^{S} \int_{\omega}|q(t)|^{2} d t\right)^{\frac{1}{2}} .
$$

Finally, in the spirit of the work of [LT06] the controllability result we have obtained yields the following relaxed observability estimate

Corollary 7.5. There exist $C_{T}>0$ and $C>0$ depending on $\Omega, \omega, T$, and $\vartheta$, such that the semi-discrete solution $q$ in $\mathscr{C}^{\infty}\left([0, T], \mathbb{C}^{\mathfrak{M}}\right)$ to

$$
\begin{cases}-\partial_{t} q+\mathcal{A}^{\mathfrak{M}} q=0 & \text { in }(0, T) \times \Omega, \\ q=0 & \text { on }(0, T) \times \partial \Omega, \\ q(T)=q_{F} \in \mathbb{C}^{\mathfrak{M}} & \end{cases}
$$

in the case $h \leq h_{0}$, satisfies

$$
|q(0)|_{L^{2}(\Omega)} \leq C_{T}\left(\int_{0}^{T} \int_{\omega}|q(t)|^{2} d t\right)^{\frac{1}{2}}+C e^{-C / h^{2}}\left|q_{F}\right|_{L^{2}(\Omega)} .
$$

Using this observability inequality, we can now provide some constructive way to compute a suitable semi-discrete control function. To this end, let $h \mapsto \phi(h) \in \mathbb{R}^{+}$be a function which tends to zero when $h$ goes to 0 and such that $e^{-C / h^{2}} / \phi(h) \rightarrow 0$. We have the following result.

TheOREM 7.6. Let $C_{T}, C$ and $h_{0}$ being the same as in Corollary 7.5.

For any mesh $\mathfrak{M}$ obtained from $\vartheta$ by (1.8) such that $h \leq h_{0}$, and any $y_{0} \in \mathbb{C}^{\mathfrak{M}}$, we consider the functional $q_{F} \in \mathbb{C}^{\mathfrak{M}} \mapsto J^{\mathfrak{M}}\left(q_{F}\right)$ defined by

$$
J^{\mathfrak{M}}\left(q_{F}\right)=\frac{1}{2} \int_{0}^{T}|q(t)|_{L^{2}(\omega)}^{2} d t+\frac{\phi(h)}{2}\left|q_{F}\right|_{L^{2}(\Omega)}^{2}+\left(y_{0}, q(0)\right)_{L^{2}(\Omega)},
$$

where $t \mapsto q(t)$ is the solution to the adjoint problem $-\partial_{t} q+\mathcal{A}^{\mathfrak{M}} q=0$ with final data $q(T)=q_{F}$.

This functional $J^{\mathfrak{m}}$ has a unique mimiser denoted by $q_{F, \text { opt }} \in \mathbb{C}^{\mathfrak{m}}$. This minimiser produces a solution $q_{\text {opt }}$ of the adjoint problem such that, if we define the control function $v(t)=\mathbf{1}_{\omega} q(t)$ then we have:

- The cost of the control is bounded as follows

$$
\int_{0}^{T}|v(t)|_{L^{2}(\omega)}^{2} d t \leq\left(C_{T}^{2}+\phi(h)\right)\left|y_{0}\right|_{L^{2}(\Omega)}^{2} .
$$

- The controlled solution y to (1.9) is such that

$$
|y(T)|_{L^{2}(\Omega)} \leq \sqrt{\phi(h)}\left(C_{T}+\sqrt{\phi(h)}\right)\left|y_{0}\right|_{L^{2}(\Omega)} .
$$


The proof of this result is not written here as it can be done along the lines of the proofs given for instance in [GL94, LT06]. Some further details will be given in [BHL09c], in connection with its fully-discrete counterpart.

Let us give some final remarks:

1. In practice, the functional $J^{\mathfrak{M}}$ is quadratic, strictly convex and coercive. Hence, the computation of $q_{F, \text { opt }}$ can be performed by using a conjugate gradient algorithm.

2. The same result holds with $\phi(h)=C e^{-C / h^{2}}$. Such a choice can be however quite unconvenient in practice as we do not know in general the value of the constant $C$ and since $e^{-C / h^{2}}$ is very likely to be smaller than machine precision for reasonable values of $h$.

3. A natural choice for $\phi$ is $\phi(h)=h^{\beta}$ with $\beta>0$ as large as desired. Minimizing $J^{\mathfrak{M}}$ we then obtain a control family that is uniformly bounded with respect to $h$ and such that the final state $y(T)$ tends to zero like $h^{\beta / 2}$. Note that the numerical scheme defined by the semi-discrete operator $\partial_{t}-\bar{D}\left(\gamma_{d} D\right)$ provides at most second-order accuracy for the computation of smooth solutions of the parabolic problem under study. A natural choice is then $\phi(h)=h^{4}$. In fact, a choice of a smaller value for $\phi(h)$ only results in a larger number of iterations of the conjugate gradient algorithm to achieve convergence.

4. As the semi-discrete controls we have obtained are bounded in $L^{2}$, then, up to a subsequence, these semi-discrete controls converge towards a function $v \in L^{2}((0, T) \times \omega)$ that actually drives the solution of the continuous parabolic problem to zero at time $T$.

5. In addition to space discretization, a time discretization can also be carried out (implicit Euler scheme or more general $\theta$-schemes). One can then observe the convergence of the fully-discrete control function to the semidiscrete control function as the time step goes to zero. See [BHL09c] for details, in particular for error estimates.

\section{Appendix A. Proofs of some technical results in Section 3.}

A.1. Proof of Lemma 3.7. For a multi-index $\delta$, by induction we have

$$
\partial^{\delta} \varphi=\lambda^{|\delta|} \nabla \psi^{\delta} \varphi+|\delta|(|\delta|-1) \lambda^{|\delta|-1} \varphi \mathcal{O}(1) .
$$

To prove (3.1) we proceed by induction on $|\alpha|+|\beta|$. The result holds for $|\alpha|=0$ and any $\beta$, and we assume it also holds in the case $|\alpha|+|\beta|=n$. In the case $|\alpha|+|\beta|=n+1$, with $|\alpha| \geq 1$, we write $\alpha=\alpha^{\prime}+\alpha^{\prime \prime}$ with $\left|\alpha^{\prime \prime}\right|=1$ and we have

$$
\begin{aligned}
\partial^{\beta}\left(r \partial^{\alpha} \rho\right) & =-s \partial^{\beta}\left(r \partial^{\alpha^{\prime}}\left(\left(\partial^{\alpha^{\prime \prime}} \varphi\right) \rho\right)\right)=-s \partial^{\beta}\left(\sum_{\delta^{\prime}+\delta^{\prime \prime}=\alpha^{\prime}}\left(\begin{array}{c}
\alpha^{\prime} \\
\delta^{\prime}
\end{array}\right)\left(\partial^{\delta^{\prime \prime}+\alpha^{\prime \prime}} \varphi\right) r \partial^{\delta^{\prime}} \rho\right) \\
& =-s \sum_{\substack{\delta^{\prime}+\delta^{\prime \prime}=\alpha^{\prime} \\
\beta^{\prime}+\beta^{\prime \prime}=\beta}}\left(\begin{array}{c}
\alpha^{\prime} \\
\delta^{\prime}
\end{array}\right)\left(\begin{array}{c}
\beta \\
\beta^{\prime}
\end{array}\right)\left(\partial^{\delta^{\prime \prime}+\beta^{\prime \prime}+\alpha^{\prime \prime}} \varphi\right) \partial^{\beta^{\prime}}\left(r \partial^{\delta^{\prime}} \rho\right) .
\end{aligned}
$$

Using the inductive hypothesis we see that the largest power in $s$ in obtained by picking $\delta^{\prime}=\alpha^{\prime}$ and $\delta^{\prime \prime}=0$ in the previous sum. The remainder is of the form $(|\alpha|-1) s^{|\alpha|-1} \mathcal{O}(1)$. The terms we selected lead to

$$
\begin{array}{r}
-s \sum_{\beta^{\prime}+\beta^{\prime \prime}=\beta}\left(\begin{array}{c}
\beta \\
\beta^{\prime}
\end{array}\right) \lambda^{\left|\beta^{\prime \prime}\right|} \varphi\left(\lambda \nabla \psi^{\beta^{\prime \prime}+\alpha^{\prime \prime}}+\left|\beta^{\prime \prime}\right| \mathcal{O}(1)\right)\left(\left|\alpha^{\prime}\right|^{\left|\beta^{\prime}\right|}(-s \varphi)^{\left|\alpha^{\prime}\right|} \lambda^{\left|\beta^{\prime}+\alpha^{\prime}\right|} \nabla \psi^{\alpha^{\prime}+\beta^{\prime}}\right. \\
\left.+\left|\alpha^{\prime}\right|\left|\beta^{\prime}\right|(s \varphi)^{\left|\alpha^{\prime}\right|} \lambda^{\left|\alpha^{\prime}+\beta^{\prime}\right|-1} \mathcal{O}(1)+\left|\alpha^{\prime}\right|\left(\left|\alpha^{\prime}\right|-1\right) s^{\left|\alpha^{\prime}\right|-1} \mathcal{O}_{\lambda}(1)\right)
\end{array}
$$


which follows as

$$
\begin{aligned}
&\left(\sum_{\beta^{\prime}+\beta^{\prime \prime}=\beta}\left(\begin{array}{c}
\beta \\
\beta^{\prime}
\end{array}\right)\left|\alpha^{\prime}\right|^{\left|\beta^{\prime}\right|}\right)(-s \varphi)^{|\alpha|} \lambda^{|\beta|+|\alpha|} \nabla \psi^{\beta+\alpha} \\
&+|\beta|(s \varphi)^{|\alpha|} \lambda^{|\alpha|+|\beta|-1} \mathcal{O}(1)+(|\alpha|-1) s^{|\alpha|-1} \mathcal{O}_{\lambda}(1)
\end{aligned}
$$

which concludes the proof of the first result, since $\sum_{\beta^{\prime}+\beta^{\prime \prime}=\beta}\left(\begin{array}{c}\beta \\ \beta^{\prime}\end{array}\right)\left|\alpha^{\prime}\right|^{\left|\beta^{\prime}\right|}=|\alpha|^{|\beta|}$. The same proof applies to $\partial^{\beta}\left(\rho \partial^{\alpha} r\right)$.

For (3.2), we first consider the case $|\alpha|=0$. We set $\nu(x, \sigma h):=r(x) \rho(x+\sigma h)$ and simply have $\nu(x, \sigma h)=e^{s(\varphi(x)-\varphi(x+\sigma h))}=e^{\mathcal{O}_{\lambda}(s h)}$, by a first-order Taylor formula, which gives the result in the case $|\beta|=0$. For $|\beta| \geq 1$, we observe that $\partial^{\beta} \nu(x, \sigma h)$ is a linear combination of terms of the form

$$
\begin{aligned}
s^{k} \partial^{\beta_{1}}(\varphi(x)-\varphi(x+\sigma h)) \cdots \partial^{\beta_{k}}(\varphi(x)-\varphi(x+\sigma h)) \nu(x, \sigma h), & \\
1 \leq k \leq|\beta|, & \beta_{1}+\cdots+\beta_{k}=\beta,
\end{aligned}
$$

which gives $\partial^{\beta} \nu(x, \sigma h)=\mathcal{O}_{\lambda}\left((s h)^{|\beta|}\right) e^{\mathcal{O}_{\lambda}(s h)}$, i.e., the result in the case $|\alpha|=0$.

Next, for $|\alpha| \geq 1$, we write $r(x)\left(\partial^{\alpha} \rho\right)(x+\sigma h)=\nu(x, \sigma h) \mu_{\alpha}(x+\sigma h)$, where we have set $\mu_{\alpha}:=r \partial^{\alpha} \rho$. By (3.1), this yields

$$
\begin{aligned}
\partial^{\beta}\left(r(x)\left(\partial^{\alpha} \rho\right)(x+\sigma h)\right) & =\sum_{\beta^{\prime}+\beta^{\prime \prime}=\beta}\left(\begin{array}{l}
\beta \\
\beta^{\prime}
\end{array}\right)\left(\partial^{\beta^{\prime}} \nu(x, \sigma h)\right)\left(\partial^{\beta^{\prime \prime}} \mu_{\alpha}(x+\sigma h)\right) \\
& =\mathcal{O}_{\lambda}\left(s^{|\alpha|}\left(1+(s h)^{|\beta|}\right)\right) e^{\mathcal{O}_{\lambda}(s h)} . \mathbf{P}
\end{aligned}
$$

A.2. Proof of Proposition 3.9. We recall that $r \rho=1$. By Lemma 3.6 we have $\tau^{+} \partial^{\alpha} \rho(x)=\partial^{\alpha} \rho(x)+C h \rho(x) \int_{0}^{1} r(x) \partial_{x} \partial^{\alpha} \rho(x+\sigma h / 2) d \sigma$, which by Lemma 3.7 yields $r \tau^{+} \partial^{\alpha} \rho=r \partial^{\alpha} \rho+s^{|\alpha|} \mathcal{O}_{\lambda}(s h) e^{\mathcal{O}_{\lambda}(s h)}=s^{|\alpha|} \mathcal{O}_{\lambda, \mathfrak{K}}(1)$. The proof is the same for $r \tau^{-} \partial^{\alpha} \rho$. For $r \mathrm{D} \rho, r \mathrm{~A} \partial^{\alpha} \rho=r \widehat{\partial^{\alpha} \rho}, r \mathrm{~A}^{2} \partial^{\alpha} \rho=r \widehat{\widehat{\partial^{\alpha}} \rho}$, and $r \mathrm{DD} \rho$ we proceed similarly, exploiting the formula in Lemma 3.6 and then applying the result of Lemma 3.7, e.g.,

$$
\begin{aligned}
\mathrm{D} \rho(x) & =\partial_{x} \rho(x)+C h^{2} \rho(x) \int_{-1}^{1}(1-|\sigma|)^{2} r(x)\left(\partial_{x}^{3} \rho\right)(x+\sigma h / 2) d \sigma \\
& =\partial_{x} \rho(x)+s \rho(x) \mathcal{O}_{\lambda, \mathfrak{K}}\left((s h)^{2}\right)=s r(x) \mathcal{O}_{\lambda, \mathfrak{K}}(1) .
\end{aligned}
$$

Noting that $\operatorname{AD} \rho=\widehat{\mathrm{D} \rho}(x)=(2 h)^{-1}(\rho(x+h)-\rho(x-h))$ we proceed as we did for $\mathrm{D} r$.

A.3. Proof of Lemma 3.10. By Lemma 3.6, we write

$$
\mathrm{D}\left(\partial^{\beta}\left(r \partial^{\alpha} \rho\right)\right)(x)=\partial_{x} \partial^{\beta}\left(r \partial^{\alpha} \rho\right)(x)+C h^{2} \int_{-1}^{1}(1-|\sigma|)^{2} \partial_{x}^{3} \partial^{\beta}\left(r \partial^{\alpha} \rho\right)(x+\sigma h / 2) d \sigma .
$$

By Lemma 3.7 we have $\partial_{x}^{3} \partial^{\beta}\left(r \partial^{\alpha} \rho\right)=\mathcal{O}_{\lambda}\left(s^{|\alpha|}\right)$, which yields the first result in the case $k=1$. For the case $k=2$, we proceed similarly, making use of the last formula listed in Lemma 3.6. For the averaging cases, we make use of the second formula in Lemma 3.6.

As in the proof of Lemma 3.7 we set $\nu(x, \sigma h):=r(x) \rho(x+\sigma h)$. We have

$$
\mathrm{D} \partial^{\beta^{\prime}} \nu(x, \sigma h)=\frac{1}{2} \int_{-1}^{1}\left(\partial_{x} \partial^{\beta^{\prime}} \nu\right)\left(x+\sigma^{\prime} h / 2, \sigma h\right) d \sigma^{\prime}=\mathcal{O}_{\lambda, \mathfrak{K}}(1), \quad\left|\beta^{\prime}\right| \leq|\beta|,
$$


for $s h \leq \mathfrak{K}$ by Lemma 3.7. Next, with $\mu_{\alpha}=r \partial^{\alpha} \rho$, we write $r(x) \partial^{\alpha} \rho(x+\sigma h)=$ $\nu(x, \sigma h) \mu_{\alpha}(x+\sigma h)$, which gives $\mathrm{D}^{\beta}\left(r(x) \partial^{\alpha} \rho(x+\sigma h)\right)$ as a linear combination of terms of the form

$$
\mathrm{A}\left(\partial^{\beta^{\prime}} \nu(., \sigma h)\right) \mathrm{D}\left(\partial^{\beta^{\prime \prime}} \mu_{\alpha}(.+\sigma h)\right)+\mathrm{D}\left(\partial^{\beta^{\prime}} \nu(., \sigma h)\right) \mathrm{A}\left(\partial^{\beta^{\prime \prime}} \mu_{\alpha}(.+\sigma h)\right), \quad \beta^{\prime}+\beta^{\prime \prime}=\beta,
$$

by the continuous and discrete Leibniz rules (Lemma 3.2). By the first part and Lemma 3.7 we have $\mathrm{D}\left(\partial^{\beta^{\prime \prime}} \mu_{\alpha}(x+\sigma h)\right)=\mathcal{O}_{\lambda, \mathfrak{K}}\left(s^{|\alpha|}\right)$. By Lemma 3.7, $\partial^{\beta^{\prime}} \nu(x, \sigma h)=$ $\mathcal{O}_{\lambda, \mathfrak{K}}(1)$ and $\partial^{\beta^{\prime \prime}} \mu_{\alpha}(x+\sigma h)=\mathcal{O}_{\lambda, \mathfrak{K}}\left(s^{|\alpha|}\right)$. The last result hence follows from (A.1). We proceed in a similar way for the case $k=2$. $\square$

A.4. Proof of Lemma 3.11. For the first two results, we proceed as in Lemma 3.10 and use Corollary 3.8.

For the last results we use the continuous and discrete Leibniz rules (Lemma 3.2) and Lemma 3.10.

A.5. Proof of Proposition 3.12. Taylor formulae yield

$$
\widehat{\mathrm{D} \rho}(x)=\frac{\rho(x+h)-\rho(x-h)}{2 h}=\partial_{x} \rho(x)+C h^{2} \int_{-1}^{1}(1-|\sigma|)^{2} \partial_{x}^{3} \rho(x+\sigma h) d \sigma,
$$

which in turn gives

$\left.\mathrm{D}^{k} \mathrm{~A}^{j} \partial^{\alpha}(r \widehat{\mathrm{D} \rho})\right)(x)=\mathrm{D}^{k} \mathrm{~A}^{j} \partial^{\alpha}\left(r \partial_{x} \rho\right)(x)+C h^{2} \int_{-1}^{1}(1-|\sigma|)^{2} \mathrm{D}^{k} \mathrm{~A}^{j} \partial^{\alpha}\left(r(x) \partial_{x}^{3} \rho(x+\sigma h)\right) d \sigma$,

and the first result follows by Lemma 3.10 (and Lemma 3.7 for the second equality).

Next, from Lemma 3.6, we write

$$
\mathrm{D}^{k}(r \mathrm{DD} \rho)(x)=\mathrm{D}^{k}\left(r \partial_{x}^{2} \rho\right)(x)+C h^{2} \int_{-1}^{1}(1-|\sigma|)^{3} \mathrm{D}^{k}\left(r(x) \partial_{x}^{4} \rho(x+\sigma h)\right) d \sigma,
$$

and the third result follows as above. For $\mathrm{D}^{k}\left(r \mathrm{~A}^{2} \rho\right)$ we use the formula for $\mathrm{A}^{2} \rho$ given in Lemma 3.6 and proceed as above.

A.6. Proof of Proposition 3.13. From (A.2) we write

$$
\begin{aligned}
\mathrm{A}^{j} \mathrm{D}^{k} \partial^{\beta}\left(r^{2}\left(\partial^{\alpha} \rho\right) \widehat{\mathrm{D} \rho}\right)(x)= & \mathrm{A}^{j} \mathrm{D}^{k} \partial^{\beta}\left(r^{2}\left(\partial^{\alpha} \rho\right) \partial_{x} \rho\right)(x) \\
& +C h^{2} \int_{-1}^{1}(1-|\sigma|)^{2} \mathrm{~A}^{j} \mathrm{D}^{k} \partial^{\beta}\left(r^{2}\left(\partial^{\alpha} \rho\right) \partial_{x}^{3} \rho(.+\sigma h)\right)(x) d \sigma,
\end{aligned}
$$

and we conclude with Lemma 3.11. For the next two results we use the formulae listed in Lemma 3.6 and proceed as above.

From Lemma 3.6, equation (A.2), and by Lemma 3.11 we have

$$
\begin{aligned}
\mathrm{A}^{j} \mathrm{D}^{k} \partial^{\alpha} & \left(r^{2} \widehat{\mathrm{D} \rho} \mathrm{D}^{2} \rho\right)=\mathrm{A}^{j} \mathrm{D}^{k} \partial^{\alpha}\left(r^{2}\left(\partial_{x} \rho\right) \partial_{x}^{2} \rho\right) \\
& +C h^{2} \int_{-1}^{1}(1-|\sigma|)^{2} \mathrm{~A}^{j} \mathrm{D}^{k} \partial^{\alpha}\left(r^{2} \partial_{x}^{3} \rho(.+\sigma h) \partial_{x}^{2} \rho\right) d \sigma \\
& +C^{\prime} h^{2} \int_{-1}^{1}(1-|\sigma|)^{3} \mathrm{~A}^{j} \mathrm{D}^{k} \partial^{\alpha}\left(r^{2}\left(\partial_{x} \rho\right) \partial_{x}^{4} \rho(.+\sigma h) d \sigma\right. \\
& +C C^{\prime} h^{4} \iint_{[-1,1]^{2}}(1-|\sigma|)^{2}\left(1-\left|\sigma^{\prime}\right|\right)^{3} \mathrm{~A}^{j} \mathrm{D}^{k} \partial^{\alpha}\left(r^{2} \partial_{x}^{3} \rho(.+\sigma h) \partial_{x}^{4} \rho\left(.+\sigma^{\prime} h\right)\right) d \sigma d \sigma^{\prime} \\
& =\partial_{x}^{k} \partial^{\alpha}\left(r^{2}\left(\partial_{x} \rho\right) \partial_{x}^{2} \rho\right)+s^{3} \mathcal{O}_{\lambda, \mathfrak{K}}\left((s h)^{2}\right) .
\end{aligned}
$$


The last result follows similarly.

Appendix B. Proofs of intermediate results in Section 4.

In this section, the calculus results of section 3 will be used and multiple averaging and difference operators will act on the weight functions and the coefficients $\xi_{1}$ and $\xi_{2}$. In the discrete setting, this in fact requires additional discretization points outside the meshes. This can be done quite naturaly since the weight functions and the coefficients are sufficiently smooth in a neighborhood of $\bar{\Omega}$.

We shall also use the notation $\mathrm{D}_{2}$ and $\mathrm{A}_{2}$ introduced in Remark 3.14 and denote by $D_{2} f$ (resp. $A_{2} f$ ) their respective actions on $\mathbb{C}^{\mathfrak{M}}$ (with extended boundary conditions).

B.1. Proof of Lemma 4.2. By Propositions 3.5 and 3.9, we have

$$
\begin{aligned}
\left|r \overline{D \rho}\left(\tau^{+} D v\right)\right|_{L^{2}(\Omega)}^{2} & =\left|\tau^{-}(r \overline{D \rho}) D v\right|_{L^{2}(\Omega)}^{2}-h(r \overline{D \rho})_{0}^{2}|D v|_{\frac{1}{2}}^{2} \\
& \leq C_{\lambda, \mathfrak{K}} s^{2}|D v|_{L^{2}(\Omega)}^{2}
\end{aligned}
$$

Similarly we have

$$
\left|r \overline{D \rho}\left(\tau^{-} D v\right)\right|_{L^{2}(\Omega)}^{2} \leq C_{\lambda, \mathfrak{K}} s^{2}|D v|_{L^{2}(\Omega)}^{2} .
$$

We also observe that

$$
\begin{aligned}
|r(\bar{D} D \rho) \overline{D v}|_{L^{2}(\Omega)}^{2} & \leq \int_{\Omega}(r \bar{D} D \rho)^{2} \overline{(D v)^{2}}=\int_{\Omega} \overline{\left.(r \bar{D} D \rho)^{2}\right)}(D v)^{2} \\
& -\frac{h}{2}(r(\bar{D} D \rho))_{0}^{2}|D v|_{\frac{1}{2}}^{2}-\frac{h}{2}(r(\bar{D} D \rho))_{N+1}^{2}|D v|_{N+\frac{1}{2}}^{2}
\end{aligned}
$$

which, by Proposition 3.9, yields

$$
|r(\bar{D} D \rho) \overline{D v}|_{L^{2}(\Omega)}^{2} \leq C_{\lambda, \mathfrak{K}} s^{4}|D v|_{L^{2}(\Omega)}^{2} .
$$

We also find

$$
|r \overline{D \rho} \overline{D v}|_{L^{2}(\Omega)}^{2} \leq C_{\lambda, \mathfrak{K}} s^{2}|D v|_{L^{2}(\Omega)}^{2} .
$$

We note that

$$
|\overline{\tilde{v}}|_{L^{2}(\Omega)}^{2} \leq \int_{\Omega} \overline{|\tilde{v}|^{2}}=|\tilde{v}|_{L^{2}(\Omega)}^{2}-\frac{h}{2}\left(|\tilde{v}|_{\frac{1}{2}}^{2}+|\tilde{v}|_{N+\frac{1}{2}}^{2}\right) \leq \int_{\Omega} \widetilde{|v|^{2}}=|v|_{L^{2}(\Omega)}^{2},
$$

by Proposition 3.5 and since $v^{\partial \mathfrak{M}}=0$. Since $\bar{D} \xi_{2_{d}}$ is bounded by $\mathrm{reg}^{0}$, by Proposition 3.9 and (B.5), we thus have

$$
\left|\left(r\left(\bar{D} \xi_{2 d}\right) \overline{D \rho}+h \mathcal{O}(1) r(\bar{D} D \rho)\right) \overline{\tilde{v}}\right|_{L^{2}(\Omega)}^{2} \leq C_{\lambda, \mathfrak{K}} s^{2}\left(1+(s h)^{2}\right)|v|_{L^{2}(\Omega)}^{2} .
$$

Similarly, since $\bar{D} \xi_{2 d}$ and $\Delta_{\xi} \varphi$ are bounded, estimates (B.1)-(B.4) and (B.6) yield the result, after an integration w.r.t. $t$. $\square$

B.2. Proof of Lemma 4.3. From the forms of $A_{1} v$ and $B_{1} v$ we have $I_{11}=$ $Q_{1}+Q_{2}+Q_{3}+Q_{4}$ with

$$
\begin{aligned}
& Q_{1}=2 \operatorname{Re} \iint_{Q} \xi_{1}^{2} r\left(\partial_{t} \rho\right)\left(\partial_{t}^{2} v\right) \partial_{t} v^{*} d t, \quad Q_{2}=2 \operatorname{Re} \iint_{Q} \xi_{1} \xi_{2} r \overline{D \rho}\left(\partial_{t}^{2} v\right) \overline{D v^{*}} d t, \\
& Q_{3}=2 \operatorname{Re} \iint_{Q} \xi_{1} r^{2}\left(\partial_{t} \rho\right) \overline{\tilde{\rho}} \bar{D}\left(\xi_{2 d} D v\right) \partial_{t} v^{*} d t, \quad Q_{4}=2 \operatorname{Re} \iint_{Q} \xi_{2} r^{2} \overline{\tilde{\rho}} \overline{D \rho} \bar{D}\left(\xi_{2 d} D v\right) \overline{D v^{*}} d t .
\end{aligned}
$$


Computation of $\boldsymbol{Q}_{\mathbf{1}}$. With $2 \operatorname{Re}\left(\partial_{t}^{2} v\right) \partial_{t} v^{*}=\partial_{t}\left|\partial_{t} v\right|^{2}$, an integration by parts yields

$$
\begin{aligned}
Q_{1} & =-\iint_{Q} \xi_{1}^{2} \partial_{t}\left(r \partial_{t} \rho\right)\left|\partial_{t} v\right|^{2} d t+\left[\int \xi_{\Omega}^{2} r\left(\partial_{t} \rho\right)\left|\partial_{t} v\right|^{2}\right]_{0}^{T_{*}} \\
& =s \lambda^{2} \iint_{Q} \xi_{1}^{2} \varphi\left(\partial_{t} \psi\right)^{2}\left|\partial_{t} v\right|^{2} d t-s \lambda\left[\int_{\Omega} \xi_{1}^{2} \varphi\left(\partial_{t} \psi\right)\left|\partial_{t} v\right|^{2}\right]_{0}^{T_{*}}+\iint_{Q} \beta_{11}^{(1)}\left|\partial_{t} v\right|^{2} d t
\end{aligned}
$$

with $\beta_{11}^{(1)}=s \lambda \varphi \mathcal{O}(1)$, by Lemma 3.7 .

Computation of $\boldsymbol{Q}_{\mathbf{2}}$. Since $\left.v\right|_{t=0}=0$, an integration by parts yields $Q_{2}=$ $Q_{2}^{(1)}+Q_{2}^{(2)}+Q_{2}^{(3)}$ with

$$
\begin{aligned}
& Q_{2}^{(1)}=-2 \operatorname{Re} \iint_{Q} \xi_{1} \xi_{2} \partial_{t}(r \overline{D \rho})\left(\partial_{t} v\right) \overline{D v^{*}} d t, \quad Q_{2}^{(2)}=-2 \operatorname{Re} \iint_{Q} \xi_{1} \xi_{2} r \overline{D \rho}\left(\partial_{t} v\right) \partial_{t} \overline{D v^{*}} d t, \\
& Q_{2}^{(3)}=2 \operatorname{Re} \int_{\Omega} \xi_{1} \xi_{2}\left(r \overline{D \rho}\left(\partial_{t} v\right) \overline{D v^{*}}\right)\left(T_{*}\right) .
\end{aligned}
$$

The last term, $Q_{2}^{(3)}$, vanishes since $\left.\psi\right|_{t=T_{*}}=$ Cst. Since $v^{\partial \mathfrak{M}}=0$, by Proposition 3.5 and Lemma 3.3 we have

$$
\begin{aligned}
& Q_{2}^{(1)}=-2 \operatorname{Re} \iint_{Q} \overline{\left(\xi_{1} \xi_{2} \partial_{t}(r \overline{D \rho})\right)} \widetilde{\partial_{t} v} D v^{*} d t-\underbrace{\frac{h^{2}}{4} \iint_{Q} D\left(\xi_{1} \xi_{2} \partial_{t}(r \overline{D \rho})\right) \partial_{t}|D v|^{2} d t}_{R_{2}^{(1)}}, \\
& Q_{2}^{(2)}=-2 \operatorname{Re} \iint_{Q} \overline{\xi_{1} \xi_{2} r \overline{D \rho}} \widetilde{\partial_{t} v} \partial_{t} D v^{*} d t-\frac{h^{2}}{2} \iint_{Q} D\left(\xi_{1} \xi_{2} r \overline{D \rho}\right)\left|D \partial_{t} v\right|^{2} d t,
\end{aligned}
$$

and, after an integration by parts w.r.t. $t$, we have

$$
R_{2}^{(1)}=\frac{h^{2}}{4} \iint_{Q} D\left(\xi_{1} \xi_{2} \partial_{t}^{2}(r \overline{D \rho})\right)|D v|^{2} d t-\frac{h^{2}}{4} \int_{\Omega} D\left(\xi_{1} \xi_{2} \partial_{t}(r \overline{D \rho})\right)\left(T_{*}\right)|D v|^{2}\left(T_{*}\right) .
$$

Since $2 \operatorname{Re} \widetilde{\partial_{t} v} \partial_{t} D v^{*}=D\left|\partial_{t} v\right|^{2}$ by Lemma 3.2, a discrete integration by parts (Proposition 3.5) yields

$$
Q_{2}^{(2)}=\iint_{Q} \overline{D\left(\xi_{1} \xi_{2} r \overline{D \rho}\right)}\left|\partial_{t} v\right|^{2} d t-\frac{h^{2}}{2} \iint_{Q} D\left(\xi_{1} \xi_{2} r \overline{D \rho}\right)\left|D \partial_{t} v\right|^{2} d t
$$

Lemma B.1. Provided $s h \leq \mathfrak{K}$, we have

$$
\begin{aligned}
& \frac{D\left(\xi_{1} \xi_{2} r \overline{D \rho}\right)}{\overline{D\left(\xi_{1} \xi_{2} r \overline{D \rho}\right)}}=-s \lambda^{2}\left(\xi_{1} \xi_{2} \varphi\left(\partial_{x} \psi\right)^{2}\right)_{d}+s \lambda \varphi_{d} \xi_{1} \xi_{2} \varphi\left(\partial_{x} \psi\right)^{2}+s \lambda \varphi \mathcal{O}(1)+s \mathcal{O}_{\lambda, \mathfrak{K}}(s h), \\
& \widehat{\xi_{1} \xi_{2} \partial_{t}(r \overline{D \rho})}=-s \lambda^{2}\left(\xi_{1} \xi_{2} \varphi\left(\partial_{t} \psi\right)\left(\partial_{x} \psi\right)\right)_{d}+s \lambda \varphi_{d} \mathcal{O}(1)+s \mathcal{O}_{\lambda, \mathfrak{K}}(s h), \\
& D\left(\xi_{1} \xi_{2} \partial_{t}^{2}(r \overline{D \rho})\right)=s \mathcal{O}_{\lambda, \mathfrak{K}}(1), \quad D\left(\xi_{1} \xi_{2} \partial_{t}(r \overline{D \rho})\right)=s \mathcal{O}_{\lambda, \mathfrak{K}}(1) .
\end{aligned}
$$


If follows that

$$
\begin{aligned}
Q_{2}= & -s \lambda^{2} \iint_{Q} \xi_{1} \xi_{2} \varphi\left(\partial_{x} \psi\right)^{2}\left|\partial_{t} v\right|^{2} d t+2 s \lambda^{2} \operatorname{Re} \iint_{Q}\left(\xi_{1} \xi_{2} \varphi\left(\partial_{t} \psi\right)\left(\partial_{x} \psi\right)\right)_{d} \widetilde{\partial_{t} v} D v^{*} d t \\
& +\iint_{Q} \gamma_{11}^{(2)}\left|D \partial_{t} v\right|^{2} d t+s h^{2} \iint_{Q} \mathcal{O}_{\lambda, \mathfrak{K}}(1)|D v|^{2} d t+s h^{2} \int_{\Omega} \mathcal{O}_{\lambda, \mathfrak{K}}(1)|D v|^{2}\left(T_{*}\right) \\
& +\iint_{Q} \beta_{11}^{(2)}\left|\partial_{t} v\right|^{2} d t+\operatorname{Re} \iint_{Q} \alpha_{11}^{(2,1)} \widetilde{\partial_{t} v} D v^{*} d t
\end{aligned}
$$

with

$$
\begin{aligned}
\gamma_{11}^{(2)} & =\frac{1}{2} h^{2} s \lambda^{2}\left(\xi_{1} \xi_{2} \varphi\left(\partial_{x} \psi\right)^{2}\right)_{d}+h^{2} s \lambda \varphi_{d} \mathcal{O}(1)+h \mathcal{O}_{\lambda, \mathfrak{K}}\left((s h)^{2}\right), \\
\beta_{11}^{(2)} & =s \lambda \varphi \mathcal{O}(1)+s \mathcal{O}_{\lambda, \mathfrak{K}}(s h), \quad \alpha_{11}^{(2,1)}=s \lambda \varphi_{d} \mathcal{O}(1)+s \mathcal{O}_{\lambda, \mathfrak{K}}(s h) .
\end{aligned}
$$

Proof of Lemma B.1. By Lemma 3.2 and Proposition 3.12, we have

$$
\begin{aligned}
D\left(\xi_{1} \xi_{2} r \overline{D \rho}\right)=D\left(\xi_{1} \xi_{2}\right) \widetilde{r \overline{D \rho}}+\widetilde{\xi_{1} \xi_{2}} D(r \overline{D \rho})= & \mathcal{O}(1)\left(\left(r \partial_{x} \rho\right)_{d}+s \mathcal{O}_{\lambda, \mathfrak{K}}\left((s h)^{2}\right)\right) \\
& +\left(\left(\xi_{1} \xi_{2}\right)_{d}+h \mathcal{O}(1)\right)\left(\left(\partial_{x}\left(r \partial_{x} \rho\right)\right)_{d}+s \mathcal{O}_{\lambda, \mathfrak{K}}\left((s h)^{2}\right)\right),
\end{aligned}
$$

which yields the second result by Lemma 3.7. We note that $\overline{D\left(\xi_{1} \xi_{2} r \overline{D \rho}\right)}=D_{2}\left(\xi_{1} \xi_{2} r \overline{D \rho}\right)$ (see Remark 3.14). We have $D_{2}\left(\xi_{1} \xi_{2} r \overline{D \rho}\right)=D_{2}\left(\xi_{1} \xi_{2}\right) A_{2}(r \overline{D \rho})+A_{2}\left(\xi_{1} \xi_{2}\right) D_{2}(r \overline{D \rho})$, and we proceed as above. The other results follow similarly. $\square$

Computation of $Q_{\mathbf{3}}$. With a discrete integration by parts (Proposition 3.5) and Lemma 3.2 , followed by an integration by parts w.r.t. $t$, we have

$$
\begin{aligned}
Q_{3}= & -2 \operatorname{Re} \iint_{Q} \xi_{2 d} D\left(\xi_{1} r^{2}\left(\partial_{t} \rho\right) \overline{\tilde{\rho}}\right) \widetilde{\partial_{t} v^{*}} D v d t+\iint_{Q} \xi_{2_{d}} \overline{\xi_{1} \partial_{t}\left(r^{2}\left(\partial_{t} \rho\right)\right.}|D v|^{2} d t \\
& \left.-\int_{\Omega} \xi_{2} \widetilde{\left(\xi_{1} r^{2}\left(\partial_{t} \rho\right)\right.} \overline{\tilde{\tilde{\rho}}}|D v|^{2}\right)\left(T_{*}\right) .
\end{aligned}
$$

Lemma B.2. Provided sh $\leq \mathfrak{K}$, we have

$$
\begin{aligned}
& D\left(\xi_{1} r^{2}\left(\partial_{t} \rho\right) \overline{\tilde{\rho}}\right)=-s \lambda^{2}\left(\xi_{1} \varphi\left(\partial_{t} \psi\right)\left(\partial_{x} \psi\right)\right)_{d}+s \lambda \varphi_{d} \mathcal{O}(1)+s \mathcal{O}_{\lambda, \mathfrak{K}}\left((s h)^{2}\right), \\
& \overline{\partial_{t}\left(\xi_{1} r^{2}\left(\partial_{t} \rho\right) \overline{\tilde{\rho}}\right)}=-s \lambda^{2}\left(\xi_{1} \varphi\left(\partial_{t} \psi\right)^{2}\right)_{d}+s \lambda \varphi_{d} \mathcal{O}(1)+s \mathcal{O}_{\lambda, \mathfrak{K}}\left((s h)^{2}\right), \\
& \overline{\xi_{1} r^{2}\left(\partial_{t} \rho\right) \overline{\tilde{\rho}}}=-s \lambda\left(\xi_{1} \varphi \partial_{t} \psi\right)_{d}+s \mathcal{O}_{\lambda, \mathfrak{K}}\left((s h)^{2}\right) .
\end{aligned}
$$

The proof follows from Lemma 3.7, Propositions 3.9 and 3.13. We thus have

$$
\begin{aligned}
Q_{3}= & 2 s \lambda^{2} \operatorname{Re} \iint_{Q}\left(\xi_{1} \xi_{2} \varphi\left(\partial_{t} \psi\right)\left(\partial_{x} \psi\right)\right)_{d} \widetilde{\partial_{t} v^{*}} D v d t-s \lambda^{2} \iint_{Q}\left(\xi_{1} \xi_{2} \varphi\left(\partial_{t} \psi\right)^{2}\right)_{d}|D v|^{2} d t \\
& +s \lambda \int_{\Omega}\left(\xi_{1} \xi_{2} \varphi \partial_{t} \psi\right)_{d}\left(T_{*}\right)|D v|^{2}\left(T_{*}\right)+\operatorname{Re} \iint_{Q} \alpha_{11}^{(3)} \widetilde{\partial_{t} v} D v^{*} d t \\
& +\iint_{Q} \nu_{11}^{(3)}|D v|^{2} d t+\int_{\Omega} \delta_{11}^{(3)}|D v|^{2}\left(T_{*}\right)
\end{aligned}
$$

where $\alpha_{11}^{(3)}=s \lambda \varphi_{d} \mathcal{O}(1)+s \mathcal{O}_{\lambda, \mathfrak{K}}\left((s h)^{2}\right), \nu_{11}^{(3)}=s \lambda \varphi_{d} \mathcal{O}(1)+s \mathcal{O}_{\lambda, \mathfrak{K}}\left((s h)^{2}\right)$, and $\delta_{11}^{(3)}=$ $s \mathcal{O}_{\lambda, \mathfrak{K}}\left((s h)^{2}\right)$. 
Computation of $\boldsymbol{Q}_{\mathbf{4}}$. By Lemmata 3.2 and 3.3, We have

$$
\begin{aligned}
Q_{4} & =-\iint_{Q} D\left(\xi_{2} \overline{\xi_{2}} r^{2} \overline{\tilde{\rho}} \overline{D \rho}\right)|D v|^{2} d t+2 \iint_{Q} \xi_{2}\left(\bar{D} \xi_{2 d}\right) r^{2} \overline{\tilde{\rho}} \overline{D \rho}|\overline{D v}|^{2} d t \\
& +\int_{0}^{T_{*}}\left(\left(\xi_{2} \overline{\xi_{2 d}} r^{2} \overline{\tilde{\rho}} \overline{D \rho}\right)_{N+1}|D v|_{N+\frac{1}{2}}^{2}-\left(\xi_{2} \overline{\xi_{2 d}} r^{2} \overline{\tilde{\rho}} \overline{D \rho}\right)_{0}|D v|_{\frac{1}{2}}^{2}\right) d t
\end{aligned}
$$

by a discrete integration by parts (Proposition 3.5).

Lemma B.3. Provided sh $\leq \mathfrak{K}$, we have

$$
\begin{aligned}
& D\left(\xi_{2} \overline{\xi_{2}} r^{2} \overline{\tilde{\rho}} \overline{D \rho}\right)=-s \lambda^{2}\left(\xi_{2}{ }^{2} \varphi\left(\partial_{x} \psi\right)^{2}\right)_{d}+s \lambda \varphi_{d} \mathcal{O}(1)+s \mathcal{O}_{\lambda, \mathfrak{K}}(s h) \\
& \xi_{2}\left(\bar{D} \xi_{2_{d}}\right) r^{2} \overline{\tilde{\rho}} \overline{D \rho}=s \lambda \varphi \mathcal{O}(1)+s \mathcal{O}_{\lambda, \mathfrak{K}}\left((s h)^{2}\right) .
\end{aligned}
$$

Since $r \overline{\tilde{\rho}}=1+\mathcal{O}_{\lambda, \mathfrak{K}}\left((s h)^{2}\right)$ by Proposition 3.9, and since $|\overline{D v}|^{2} \leq \overline{|D v|^{2}}$, it follows that we have

$$
\begin{aligned}
Q_{4} \geq & s \lambda^{2} \iint_{Q}\left(\xi_{2}^{2} \varphi\left(\partial_{x} \psi\right)^{2}\right)_{d}|D v|^{2} d t+\iint_{Q} \nu_{11}^{(4)}|D v|^{2} d t \\
& +\int_{0}^{T_{*}}\left(1+\mathcal{O}_{\lambda, \mathfrak{K}}\left((s h)^{2}\right)\right)\left(\left(\xi_{2} \overline{\xi_{2}} r \overline{D \rho}\right)_{N+1}|D v|_{N+\frac{1}{2}}^{2}-\left(\xi_{2} \overline{\xi_{2 d}} r \overline{D \rho}\right)_{0}|D v|_{\frac{1}{2}}^{2}\right) d t
\end{aligned}
$$

where $\nu_{11}^{(4)}=s \lambda \varphi_{d} \mathcal{O}(1)+s \mathcal{O}_{\lambda, \mathfrak{K}}(s h)$.

Proof of Lemma B.3. By Lemma 3.3, and Proposition 3.13 we write

$$
\begin{aligned}
D\left(\xi_{2} \overline{\xi_{2 d}}\right. & r^{2} \\
& \overline{\tilde{\rho}} \overline{D \rho})=D\left(\xi_{2} \overline{\xi_{2 d}}\right) \widetilde{r^{2} \overline{\tilde{\rho}} \overline{D \rho}}+\widetilde{\xi_{2} \overline{\xi_{2 d}}} D\left(r^{2} \overline{\tilde{\rho}} \overline{D \rho}\right) \\
& =\mathcal{O}(1)\left(r \partial_{x} \rho+s \mathcal{O}_{\lambda, \mathfrak{K}}\left((s h)^{2}\right)\right)_{d}+\left(\xi_{2 d}^{2}+h \mathcal{O}(1)\right)\left(\partial_{x}\left(r \partial_{x} \rho\right)+s \mathcal{O}_{\lambda, \mathfrak{K}}\left((s h)^{2}\right)\right)_{d},
\end{aligned}
$$

and the first result follows from Lemma 3.7. The second result follows from Lemma 3.7 and Proposition 3.9.

Gathering of the different terms. The results obtained in (B.7)-(B.10) yield

$$
I_{11} \geq I_{11}^{a}+I_{11}^{b}+W_{11}+Y_{11}-\tilde{X}_{11}-J_{11},
$$

where $I_{11}^{a}, W_{11}, Y_{11}$, and $J_{11}$ are as given in the statement of Lemma $4.3, \tilde{X}_{11}$ has the same form as $X_{11}$ in the statement of Lemma 4.3, and

$$
\begin{aligned}
I_{11}^{b}=-s \lambda^{2} \frac{h^{2}}{2} \iint_{Q}\left(\varphi \xi_{1}^{2}\left(\partial_{t} \psi\right)^{2}\right)_{d}\left|D \partial_{t} v\right|^{2} d t+2 s \lambda^{2} \int_{0}^{T_{*}}\left[\int_{\Omega} \xi_{1}^{2} \varphi\left(\partial_{t} \psi\right)^{2}\left|\partial_{t} v\right|^{2}\right. \\
\left.\quad+\int_{\Omega}\left(\xi_{2}{ }^{2} \varphi\left(\partial_{x} \psi\right)^{2}\right)_{d}|D v|^{2}+2 \operatorname{Re} \int_{\Omega}\left(\xi_{1} \xi_{2} \varphi\left(\partial_{t} \psi\right)\left(\partial_{x} \psi\right)\right)_{d} \widetilde{\partial_{t} v} D v^{*}\right] d t .
\end{aligned}
$$

Note that the first term in $I_{11}^{b}$ comes from the fact that we added exactly the opposite term in $W_{11}$ in order to ensure that $W \geq 0$ (see Lemma 4.7 and its proof). We conclude the proof of Lemma 4.3 with the following lemma. $\square$

Lemma B.4. Provided sh $\leq \mathfrak{K}$, we have $I_{11}^{b} \geq \iint_{Q} \mathcal{O}_{\lambda}(s h)\left|\partial_{t} v\right|^{2} d t$.

Proof. We write

$$
I_{11}^{b}=2 s \lambda^{2} \iint_{Q} \varphi_{d}\left|\xi_{1}\left(\partial_{t} \psi\right)_{d} \widetilde{\partial_{t}} v+\left(\xi_{2} \partial_{x} \psi\right)_{d} D v\right|^{2} d t+2 s \lambda^{2} \int_{0}^{T_{*}} L(t) d t \geq 2 s \lambda^{2} \int_{0}^{T_{*}} L(t) d t,
$$


with

$$
\begin{aligned}
L(t) & =\int_{\Omega} \varphi \xi_{1}^{2}\left(\partial_{t} \psi\right)^{2}\left|\partial_{t} v\right|^{2}-\left.\int_{\Omega}\left(\varphi \xi_{1}^{2}\left(\partial_{t} \psi\right)^{2}\right)_{d}\left|\widetilde{\left.\partial_{t} v\right|^{2}}-\frac{h^{2}}{4} \int_{\Omega}\left(\xi_{1}^{2} \varphi\left(\partial_{t} \psi\right)^{2}\right)_{d}\right| D \partial_{t} v\right|^{2} d t \\
& =\int_{\Omega} \xi_{1}^{2} \varphi\left(\partial_{t} \psi\right)^{2}\left|\partial_{t} v\right|^{2}-\int_{\Omega}\left(\xi_{1}^{2} \varphi\left(\partial_{t} \psi\right)^{2}\right)_{d} \widehat{\left.\partial_{t} v\right|^{2}} \\
& =\int_{\Omega}\left(\xi_{1}^{2} \varphi\left(\partial_{t} \psi\right)^{2}-\overline{\left(\xi_{1}^{2} \varphi\left(\partial_{t} \psi\right)^{2}\right)_{d}}\right)\left|\partial_{t} v\right|^{2},
\end{aligned}
$$

by Lemma 3.3 and Proposition 3.5 as $v^{\partial \mathfrak{M}}=0$. We conclude since $\xi_{1}^{2} \varphi\left(\partial_{t} \psi\right)^{2}-$ $\overline{\left(\xi_{1}^{2} \varphi\left(\partial_{t} \psi\right)^{2}\right)_{d}}=h \mathcal{O}_{\lambda}(1)$ by Lemma 3.6.

B.3. Proof of Lemma 4.4. From the forms of $A_{1} v$ and $B_{2} v$ we have $I_{12}=$ $Q_{1}+Q_{2}$ with

$$
Q_{1}=-2 s \operatorname{Re} \iint_{Q} \xi_{1}\left(\Delta_{\xi} \varphi\right) v^{*} \partial_{t}^{2} v d t \quad \text { and } \quad Q_{2}=-2 s \operatorname{Re} \iint_{Q} r \overline{\tilde{\rho}}\left(\Delta_{\xi} \varphi\right) v^{*} \bar{D}\left(\xi_{2 d} D v\right) d t
$$

With an integration by parts w.r.t. $t$ we obtain $Q_{1}=2 s \iint_{Q} \xi_{1}\left(\Delta_{\xi} \varphi\right)\left|\partial_{t} v\right|^{2} d t+R_{1}$, where

$$
\begin{aligned}
R_{1} & =2 s \operatorname{Re} \iint_{Q} \xi_{1} \partial_{t}\left(\Delta_{\xi} \varphi\right) v^{*} \partial_{t} v d t-2 s \operatorname{Re} \int_{\Omega} \xi_{1}\left(\Delta_{\xi} \varphi\right)\left(T_{*}\right) v^{*}\left(T_{*}\right) \partial_{t} v\left(T_{*}\right) \\
& =s \iint_{Q} \mathcal{O}_{\lambda}(1)|v|^{2} d t+s \int_{\Omega} \mathcal{O}_{\lambda}(1)|v|^{2}\left(T_{*}\right)+\operatorname{Re} \int_{\Omega} \mathcal{O}_{\lambda}(1) v^{*}\left(T_{*}\right) \partial_{t} v\left(T_{*}\right),
\end{aligned}
$$

using $2 \operatorname{Re} v^{*} \partial_{t} v=\partial_{t}|v|^{2}$, and an additional integration by parts w.r.t. $t$, since $\xi_{1} \partial_{t}\left(\Delta_{\xi} \varphi\right)=\mathcal{O}_{\lambda}(1), \xi_{1} \partial_{t}^{2}\left(\Delta_{\xi} \varphi\right)=\mathcal{O}_{\lambda}(1)$ and $\xi_{1} \Delta_{\xi} \varphi\left(T_{*}\right)=\mathcal{O}_{\lambda}(1)$.

For concision we now set $q=r \overline{\tilde{\rho}}\left(\Delta_{\xi} \varphi\right)$. For the term $Q_{2}$, a discrete integration by parts gives

$$
Q_{2}=2 s \iint_{Q} \tilde{q} \xi_{2 d}|D v|^{2} d t+2 s \operatorname{Re} \iint_{Q}(D q) \xi_{2 d} \tilde{v}^{*} D v d t
$$

Since by Proposition 3.9 we have $q=\Delta_{\xi} \varphi+\mathcal{O}_{\lambda, \mathfrak{K}}\left((s h)^{2}\right)$, then

$$
\tilde{q}=\left(\Delta_{\xi} \varphi\right)_{d}+\mathcal{O}_{\lambda, \mathfrak{K}}\left(h+(s h)^{2}\right)
$$

as $\widetilde{\Delta_{\xi} \varphi}=\left(\Delta_{\xi} \varphi\right)_{d}+\mathcal{O}_{\lambda}(h)$ since $\operatorname{reg}(\xi) \leq \operatorname{reg}^{0}$. We note also that

$$
D q=D(r \overline{\tilde{\rho}}) \widetilde{\Delta_{\xi} \varphi}+\widetilde{(r \overline{\tilde{\rho}})} D\left(\Delta_{\xi} \varphi\right)=\mathcal{O}_{\lambda, \mathfrak{K}}(1),
$$

by Propositions 3.9 and 3.12. We thus obtain $Q_{2}=2 s \iint_{Q}\left(\xi_{2} \Delta_{\xi} \varphi\right)_{d}|D v|^{2} d t+R_{2}$, with

$$
R_{2}=s \iint_{Q} \mathcal{O}_{\lambda, \mathfrak{K}}\left(h+(s h)^{2}\right)|D v|^{2} d t+s \operatorname{Re} \iint_{Q} \mathcal{O}_{\lambda, \mathfrak{K}}(1) \tilde{v}^{*} D v d t
$$

Observing that

$$
\Delta_{\xi} \varphi=\lambda^{2}\left|\nabla_{\xi} \psi\right|^{2} \varphi+\lambda \varphi \mathcal{O}(1),
$$

by Lemma 3.7, we obtain the desired result. 
B.4. Proof of Lemma 4.5. From the forms of $A_{2} v$ and $B_{1} v$ we have $I_{21}=$ $Q_{1}+Q_{2}+Q_{3}+Q_{4}$ with

$$
\begin{aligned}
& Q_{1}=2 \operatorname{Re} \iint_{Q} \xi_{1}^{2} r^{2}\left(\partial_{t}^{2} \rho\right)\left(\partial_{t} \rho\right) v \partial_{t} v^{*} d t, \quad Q_{2}=2 \operatorname{Re} \iint_{Q} \xi_{1} r^{2}\left(\partial_{t}^{2} \rho\right) \overline{D \rho} \xi_{2} v \overline{D v^{*}} d t \\
& Q_{3}=2 \operatorname{Re} \iint_{Q} \xi_{1} \xi_{2} r^{2}(\bar{D} D \rho)\left(\partial_{t} \rho\right) \overline{\tilde{v}} \partial_{t} v^{*} d t, \quad Q_{4}=2 \operatorname{Re} \iint_{Q} \xi_{2}{ }^{2} r^{2}(\bar{D} D \rho) \overline{D \rho} \overline{\tilde{v}} \overline{D v^{*}} d t
\end{aligned}
$$

Computation of $\boldsymbol{Q}_{\mathbf{1}}$. We set $q_{1}=\xi_{1}^{2} r^{2}\left(\partial_{t}^{2} \rho\right)\left(\partial_{t} \rho\right)$. With an integration by parts, we have

$$
\begin{aligned}
Q_{1}= & \iint_{Q} q_{1} \partial_{t}|v|^{2} d t=-\iint_{Q}\left(\partial_{t} q_{1}\right)|v|^{2} d t+\int_{\Omega} q_{1}\left(T_{*}\right)|v|^{2}\left(T_{*}\right) \\
= & 3 s^{3} \lambda^{4} \iint_{Q} \xi_{1}^{2} \varphi^{3}\left(\partial_{t} \psi\right)^{4}|v|^{2} d t-(s \lambda)^{3} \int_{\Omega} \xi_{1}^{2}\left(\varphi \partial_{t} \psi\right)^{3}\left(T_{*}\right)|v|^{2}\left(T_{*}\right) \\
& +\iint_{Q} \mu_{21}^{(1)}|v|^{2} d t+\int_{\Omega} \eta_{21}^{(1)}|v|^{2}\left(T_{*}\right),
\end{aligned}
$$

by Corollary 3.8, where $\mu_{21}^{(1)}=(s \lambda \varphi)^{3} \mathcal{O}(1)+s^{2} \mathcal{O}_{\lambda}(1)$ and $\eta_{21}^{(1)}=s^{2} \mathcal{O}_{\lambda}(1)$.

Computation of $\boldsymbol{Q}_{\mathbf{2}}$. We set $q_{2}=\xi_{1} \xi_{2} r^{2}\left(\partial_{t}^{2} \rho\right) \overline{D \rho}$. We have

$$
\begin{aligned}
Q_{2} & =2 \operatorname{Re} \iint_{Q} \widetilde{q_{2} v} D v^{*} d t=\iint_{Q} \tilde{q}_{2} D|v|^{2} d t+\frac{h^{2}}{2} \iint_{Q} D q_{2}|D v|^{2} d t \\
& =-\iint_{Q} \bar{D} \tilde{q}_{2}|v|^{2} d t+\frac{h^{2}}{2} \iint_{Q} D q_{2}|D v|^{2} d t
\end{aligned}
$$

by Proposition 3.5 and Lemmata 3.2 and 3.3, using $v^{\partial \mathfrak{M}}=0$.

Lemma B.5. Provided $s h \leq \mathfrak{K}$, we have $D q_{2}=s^{3} \mathcal{O}_{\lambda, \mathfrak{K}}(1)$ and

$\bar{D} \tilde{q}_{2}=\overline{D q_{2}}=-3(s \varphi)^{3} \lambda^{4} \xi_{1}\left(\partial_{t} \psi\right)^{2} \xi_{2}\left(\partial_{x} \psi\right)^{2}+(s \lambda \varphi)^{3} \mathcal{O}(1)+s^{2} \mathcal{O}_{\lambda}(1)+s^{3} \mathcal{O}_{\lambda, \mathfrak{K}}\left((s h)^{2}\right)$.

It follows that

$$
Q_{2}=3 s^{3} \lambda^{4} \iint_{Q} \varphi^{3} \xi_{1}\left(\partial_{t} \psi\right)^{2} \xi_{2}\left(\partial_{x} \psi\right)^{2}|v|^{2} d t+\iint_{Q} \mu_{21}^{(2)}|v|^{2} d t+\iint_{Q} \nu_{21}^{(2)}|D v|^{2} d t
$$

with $\mu_{21}^{(2)}=(s \lambda \varphi)^{3} \mathcal{O}(1)+s^{2} \mathcal{O}_{\lambda}(1)+s^{3} \mathcal{O}_{\lambda, \mathfrak{K}}\left((s h)^{2}\right)$ and $\nu_{21}^{(2)}=s \mathcal{O}_{\lambda, \mathfrak{K}}\left((s h)^{2}\right)$.

Proof of Lemma B.5. We write

$$
\begin{aligned}
D q_{2}=( & \left.D\left(\xi_{1} \xi_{2}\right)\right) \overline{r^{2}\left(\partial_{t}^{2} \rho\right) \overline{\overline{D \rho}}}+\widetilde{\xi_{1} \xi_{2}} D\left(r^{2}\left(\partial_{t}^{2} \rho\right) \overline{D \rho}\right)=\mathcal{O}(1)\left(\left(r^{2}\left(\partial_{t}^{2} \rho\right) \partial_{x} \rho\right)_{d}+s^{3} \mathcal{O}_{\lambda, \mathfrak{K}}\left((s h)^{2}\right)\right) \\
& +\left(\left(\xi_{1} \xi_{2}\right)_{d}+h \mathcal{O}(1)\right)\left(\left(\partial_{x}\left(r^{2}\left(\partial_{t}^{2} \rho\right) \partial_{x} \rho\right)\right)_{d}+s^{3} \mathcal{O}_{\lambda, \mathfrak{K}}\left((s h)^{2}\right)\right)
\end{aligned}
$$

by Lemmata 3.2 and 3.13. Since

$$
\begin{aligned}
& r^{2}\left(\partial_{t}^{2} \rho\right) \partial_{x} \rho=-(s \lambda \varphi)^{3}\left(\partial_{t} \psi\right)^{2}\left(\partial_{x} \psi\right)+s^{2} \mathcal{O}_{\lambda}(1) \\
& \begin{aligned}
\partial_{x}\left(r^{2}\left(\partial_{t}^{2} \rho\right) \partial_{x} \rho\right) & =-3(s \varphi)^{3} \lambda^{4}\left(\partial_{t} \psi\right)^{2}\left(\partial_{x} \psi\right)^{2}+s^{2} \mathcal{O}_{\lambda}(1)+(s \lambda \varphi)^{3} \mathcal{O}(1) \\
& =s^{3} \mathcal{O}_{\lambda}(1),
\end{aligned}
\end{aligned}
$$


by Corollary 3.8, we have

$$
D q_{2}=-3(s \varphi)^{3} \lambda^{4} \xi_{1}\left(\partial_{t} \psi\right)^{2} \xi_{2}\left(\partial_{x} \psi\right)^{2}+(s \lambda \varphi)^{3} \mathcal{O}(1)+s^{2} \mathcal{O}_{\lambda}(1)+s^{3} \mathcal{O}_{\lambda, \mathfrak{K}}\left((s h)^{2}\right),
$$

and the first result follows. We note that $\overline{D q_{2}}=D_{2} q_{2}$ (see Remark 3.14). We have

$$
\overline{D q_{2}}=\left(D_{2}\left(\xi_{1} \xi_{2}\right)\right) A_{2}\left(r^{2}\left(\partial_{t}^{2} \rho\right) \overline{D \rho}\right)+\left(A_{2}\left(\xi_{1} \xi_{2}\right)\right) D_{2}\left(r^{2}\left(\partial_{t}^{2} \rho\right) \overline{D \rho}\right) .
$$

Using Remark 3.14, proceeding as above we obtain the second result.

Computation of $\boldsymbol{Q}_{\mathbf{3}}$. We set $q_{3}=\xi_{1} \xi_{2} r^{2}(\bar{D} D \rho)\left(\partial_{t} \rho\right)$. By Proposition 3.5 and Lemma 3.3, we then have

$$
Q_{3}=2 \operatorname{Re} \iint_{Q} \widetilde{q_{3} \partial_{t} v^{*}} \tilde{v} d t=\underbrace{\iint_{Q} \tilde{q}_{3} \partial_{t}|\tilde{v}|^{2} d t}_{Q_{3}^{(1)}}+\underbrace{\frac{h^{2}}{2} \operatorname{Re} \iint_{Q}\left(D q_{3}\right)\left(D \partial_{t} v^{*}\right) \tilde{v} d t}_{Q_{3}^{(2)}},
$$

Lemma B.6. Provided $s h \leq \mathfrak{K}$, we have $D q_{3}=s^{3} \mathcal{O}_{\lambda, \mathfrak{K}}(1)$.

By Young inequalities, we have the following estimate

$$
\left|Q_{3}^{(2)}\right| \leq C h^{2} s \iint_{Q}\left|D \partial_{t} v\right|^{2} d t+s^{3}(s h)^{2} \iint_{Q} \mathcal{O}_{\lambda, \mathfrak{K}}(1)|v|^{2} d t,
$$

since $|\tilde{v}|^{2} \leq \widetilde{|v|^{2}}$ and then exploiting Proposition 3.5 and $v^{\partial \mathfrak{M}}=0$. Next, with an integration by parts, we see that

$$
\begin{aligned}
Q_{3}^{(1)} & =-\iint_{Q}\left(\partial_{t} \tilde{q}_{3}\right)|\tilde{v}|^{2} d t+\int_{\Omega} \tilde{q}_{3}\left(T_{*}\right)|\tilde{v}|^{2}\left(T_{*}\right) \\
& =-\iint_{Q}\left(\partial_{t} \tilde{q}_{3}\right) \widetilde{|v|^{2}} d t+\int_{\Omega} \tilde{q}_{3}\left(T_{*}\right) \widetilde{|v|^{2}}\left(T_{*}\right)-\frac{h^{2}}{4} \iint_{Q}\left(\partial_{t} \tilde{q}_{3}\right)|D v|^{2} d t-\frac{h^{2}}{4} \int_{\Omega} \tilde{q}_{3}\left(T_{*}\right)|D v|^{2}\left(T_{*}\right) \\
& =-\iint_{Q}\left(\partial_{t} \overline{\tilde{q}}_{3}\right)|v|^{2} d t+\int_{\Omega} \overline{\tilde{q}}_{3}\left(T_{*}\right)|v|^{2}\left(T_{*}\right)-\frac{h^{2}}{4} \iint_{Q}\left(\partial_{t} \tilde{q}_{3}\right)|D v|^{2} d t-\frac{h^{2}}{4} \int_{\Omega} \tilde{q}_{3}\left(T_{*}\right)|D v|^{2}\left(T_{*}\right),
\end{aligned}
$$

by Lemma 3.3 and Proposition 3.5, using $v^{\partial \mathfrak{M}}=0$.

Lemma B.7. We have

$$
\begin{aligned}
& \tilde{q}_{3}=s^{3} \mathcal{O}_{\lambda, \mathfrak{K}}(1), \quad \partial_{t} \tilde{q}_{3}=s^{3} \mathcal{O}_{\lambda, \mathfrak{K}}(1), \\
& \overline{\tilde{q}}_{3}=-(s \lambda \varphi)^{3} \xi_{1}\left(\partial_{t} \psi\right) \xi_{2}\left(\partial_{x} \psi\right)^{2}+s^{3} \mathcal{O}_{\lambda, \mathfrak{K}}\left((s h)^{2}\right)+s^{2} \mathcal{O}_{\lambda, \mathfrak{K}}(1), \\
& \partial_{t} \overline{\tilde{q}}_{3}=-3 s^{3} \lambda^{4} \varphi^{3} \xi_{1}\left(\partial_{t} \psi\right)^{2} \xi_{2}\left(\partial_{x} \psi\right)^{2}+(s \lambda \varphi)^{3} \mathcal{O}(1)+s^{2} \mathcal{O}_{\lambda}(1)+s^{3} \mathcal{O}_{\lambda, \mathfrak{K}}\left((s h)^{2}\right) .
\end{aligned}
$$

We have thus obtained,

$$
\begin{aligned}
Q_{3} \geq & 3 s^{3} \lambda^{4} \iint_{Q} \varphi^{3} \xi_{1}\left(\partial_{t} \psi\right)^{2} \xi_{2}\left(\partial_{x} \psi\right)^{2}|v|^{2} d t-(s \lambda)^{3} \int_{\Omega}\left(\varphi^{3} \xi_{1}\left(\partial_{t} \psi\right) \xi_{2}\left(\partial_{x} \psi\right)^{2}\right)\left(T_{*}\right)|v|^{2}\left(T_{*}\right) \\
& +\iint_{Q} \mu_{21}^{(3)}|v|^{2} d t+\int_{\Omega} \eta_{21}^{(3)}|v|^{2}\left(T_{*}\right)+\iint_{Q} \nu_{21}^{(3)}|D v|^{2} d t+\int_{\Omega} \delta_{21}^{(3)}|D v|^{2}\left(T_{*}\right) \\
& +\iint_{Q} \gamma_{21}\left|D \partial_{t} v\right|^{2} d t
\end{aligned}
$$


where

$$
\begin{aligned}
& \mu_{21}^{(3)}=(s \lambda \varphi)^{3} \mathcal{O}(1)+s^{2} \mathcal{O}_{\lambda}(1)+s^{3} \mathcal{O}_{\lambda, \mathfrak{K}}\left((s h)^{2}\right), \quad \eta_{21}^{(3)}=s^{3} \mathcal{O}_{\lambda, \mathfrak{K}}\left((s h)^{2}\right)+s^{2} \mathcal{O}_{\lambda, \mathfrak{K}}(1), \\
& \nu_{21}^{(3)}=s \mathcal{O}_{\lambda, \mathfrak{K}}\left((s h)^{2}\right), \quad \text { and } \delta_{21}^{(3)}=s \mathcal{O}_{\lambda, \mathfrak{K}}\left((s h)^{2}\right), \quad \gamma_{21}=h \mathcal{O}(s h) .
\end{aligned}
$$

Proof of Lemma B.6. We have

$$
D q_{3}=\left(D\left(\xi_{1} \xi_{2}\right)\right) \widetilde{r^{2}(\bar{D} D \rho)\left(\partial_{t} \rho\right)}+\widetilde{\xi_{1} \xi_{2}} D\left(r^{2}(\bar{D} D \rho)\left(\partial_{t} \rho\right)\right)=s^{3} \mathcal{O}_{\lambda, \mathfrak{K}}(1),
$$

by Proposition 3.13 and Corollary 3.8 , since $D\left(\xi_{1} \xi_{2}\right)$ is bounded.

Proof of Lemma B.7. From Proposition 3.13 we have

$$
q_{3}=\xi_{1} \xi_{2} r^{2}\left(\partial_{x}^{2} \rho\right) \partial_{t} \rho+s^{3} \mathcal{O}_{\lambda, \mathfrak{K}}\left((s h)^{2}\right), \quad \partial_{t} q_{3}=\xi_{1} \xi_{2} \partial_{t}\left(r^{2}\left(\partial_{x}^{2} \rho\right) \partial_{t} \rho\right)+s^{3} \mathcal{O}_{\lambda, \mathfrak{K}}\left((s h)^{2}\right) .
$$

By Lemma 3.3, we now write

$$
\begin{aligned}
\tilde{q}_{3} & =\widetilde{\xi_{1} \xi_{2}} \overline{r^{2}\left(\partial_{x}^{2} \rho\right) \partial_{t} \rho}+\frac{h^{2}}{4}\left(D\left(\xi_{1} \xi_{2}\right)\right) D\left(r^{2}\left(\partial_{x}^{2} \rho\right) \partial_{t} \rho\right)+s^{3} \mathcal{O}_{\lambda, \mathfrak{K}}\left((s h)^{2}\right) \\
& =\left(\left(\xi \xi_{2}\right)_{d}+h \mathcal{O}(1)\right)\left(\left(r^{2}\left(\partial_{x}^{2} \rho\right) \partial_{t} \rho\right)_{d}+s^{3} h^{2} \mathcal{O}_{\lambda, \mathfrak{K}}(1)\right)+h^{2} \mathcal{O}(1) \mathcal{O}_{\lambda, \mathfrak{K}}\left(s^{3}\right)+s^{3} \mathcal{O}_{\lambda, \mathfrak{K}}\left((s h)^{2}\right) \\
& =\left(\xi_{1} \xi_{2} r^{2}\left(\partial_{x}^{2} \rho\right) \partial_{t} \rho\right)_{d}+s^{3} \mathcal{O}_{\lambda, \mathfrak{K}}\left((s h)^{2}\right)+s^{2} \mathcal{O}_{\lambda, \mathfrak{K}}(1)
\end{aligned}
$$

by Lemma 3.11 and Corollary 3.8. Similarly, we find

$$
\partial_{t} \tilde{q}_{3}=\left(\xi_{1} \xi_{2} \partial_{t}\left(r^{2}\left(\partial_{x}^{2} \rho\right) \partial_{t} \rho\right)\right)_{d}+s^{3} \mathcal{O}_{\lambda, \mathfrak{K}}\left((s h)^{2}\right)+s^{2} \mathcal{O}_{\lambda, \mathfrak{K}}(1)
$$

Iterating the averaging procedure we obtain similar estimates for $\overline{\tilde{q}}_{3}$ and $\partial_{t} \overline{\tilde{q}}_{3}$ (sampled on the primal mesh) and we conclude with Corollary 3.8. $\square$

Computation of $\boldsymbol{Q}_{\mathbf{4}}$. We set $q_{4}=\xi_{2}{ }^{2} r^{2}(\bar{D} D \rho) \overline{D \rho}$. Observing that $\overline{D v^{*}}=\bar{D} \tilde{v}^{*}$, we have

$$
Q_{4}=\iint_{Q} q_{4} \bar{D}|\tilde{v}|^{2} d t=\underbrace{-\iint_{Q}\left(D q_{4}\right)|\tilde{v}|^{2} d t}_{Q_{4}^{(1)}}+\underbrace{\int_{0}^{T_{*}}\left(\left(q_{4}\right)_{N+1}\left|\tilde{v}_{N+\frac{1}{2}}\right|^{2}-\left(q_{4}\right)_{0}\left|\tilde{v}_{\frac{1}{2}}\right|^{2}\right) d t}_{Q_{4}^{(2)}},
$$

by Lemma 3.2 and Proposition 3.5. We note that $\tilde{v}_{\frac{1}{2}}=\frac{h}{2}(D v)_{\frac{1}{2}}$ and $\tilde{v}_{N+\frac{1}{2}}=$ $-\frac{h}{2}(D v)_{N+\frac{1}{2}}$. By Proposition 3.9 we have $q_{4}=s^{2} \mathcal{O}_{\lambda, \mathfrak{K}}(1) r \overline{D \rho}$. It follows that

$$
Q_{4}^{(2)}=(s h)^{2} \int_{0}^{T_{*}}\left(\mathcal{O}_{\lambda, \mathfrak{K}}(1)(r \overline{D \rho})_{0}|D v|_{\frac{1}{2}}^{2}+\mathcal{O}_{\lambda, \mathfrak{K}}(1)(r \overline{D \rho})_{N+1}|D v|_{N+\frac{1}{2}}^{2}\right) d t
$$

Next, by Lemma 3.3 and Proposition 3.5, we write

$$
\begin{aligned}
Q_{4}^{(1)} & =-\iint_{Q}\left(D q_{4}\right) \widetilde{|v|^{2}} d t+\frac{h^{2}}{4} \iint_{Q}\left(D q_{4}\right)|D v|^{2} d t \\
& =-\iint_{Q} \overline{D q_{4}}|v|^{2} d t+\frac{h^{2}}{4} \iint_{Q}\left(D q_{4}\right)|D v|^{2} d t .
\end{aligned}
$$

Lemma B.8. Provided $s h \leq \mathfrak{K}$, we have $D q_{4}=s^{3} \mathcal{O}_{\lambda, \mathfrak{K}}(1)$ and

$$
\overline{D q_{4}}=-s^{3} \lambda^{4} \varphi^{3} \xi_{2}^{2}\left(\partial_{x} \psi\right)^{4}+(s \lambda \varphi)^{3} \mathcal{O}(1)+s^{2} \mathcal{O}_{\lambda, \mathfrak{K}}(1)+s^{3} \mathcal{O}_{\lambda, \mathfrak{K}}\left((s h)^{2}\right) .
$$


We have thus obtained

$$
\begin{aligned}
Q_{4}= & 3 s^{3} \lambda^{4} \iint_{Q} \varphi^{3} \xi_{2}^{2}\left(\partial_{x} \psi\right)^{4}|v|^{2} d t+\iint_{Q} \mu_{21}^{(4)}|v|^{2} d t+\iint_{Q} \nu_{21}^{(4)}|D v|^{2} d t \\
& +\int_{0}^{T_{*}}\left(\mathcal{O}_{\lambda, \mathfrak{K}}\left((s h)^{2}\right)(r \overline{D \rho})_{0}|D v|_{\frac{1}{2}}^{2}+\mathcal{O}_{\lambda, \mathfrak{K}}\left((s h)^{2}\right)(r \overline{D \rho})_{N+1}|D v|_{N+\frac{1}{2}}^{2}\right) d t,
\end{aligned}
$$

where

$$
\mu_{21}^{(4)}=(s \lambda \varphi)^{3} \mathcal{O}(1)+s^{2} \mathcal{O}_{\lambda, \mathfrak{K}}(1)+s^{3} \mathcal{O}_{\lambda, \mathfrak{K}}\left((s h)^{2}\right), \quad \nu_{21}^{(4)}=s \mathcal{O}_{\lambda, \mathfrak{K}}\left((s h)^{2}\right) .
$$

Proof of Lemma B.8. By Proposition 3.13 we have

$$
\begin{aligned}
D q_{4}= & D\left(\xi_{2}{ }^{2}\right) \overline{r^{2}(\bar{D} D \rho) \overline{D \rho}}+\widetilde{\xi_{2}^{2}} D\left(r^{2}(\bar{D} D \rho) \overline{D \rho}\right) \\
= & \mathcal{O}(1)\left(\left(r^{2}\left(\partial_{x}^{2} \rho\right) \partial_{x} \rho\right)_{d}+s^{3} \mathcal{O}_{\lambda, \mathfrak{K}}\left((s h)^{2}\right)\right) \\
& \quad+\left(\xi_{2}^{2}+h \mathcal{O}(1)\right)\left(\left(\partial_{x}\left(r^{2}\left(\partial_{x}^{2} \rho\right) \partial_{x} \rho\right)\right)_{d}+s^{3} \mathcal{O}_{\lambda, \mathfrak{K}}\left((s h)^{2}\right)\right) \\
= & \xi_{2}^{2}\left(\partial_{x}\left(r^{2}\left(\partial_{x}^{2} \rho\right) \partial_{x} \rho\right)\right)_{d}+(s \lambda \varphi)_{d}^{3} \mathcal{O}(1)+s^{2} \mathcal{O}_{\lambda, \mathfrak{K}}(1)+s^{3} \mathcal{O}_{\lambda, \mathfrak{K}}\left((s h)^{2}\right) .
\end{aligned}
$$

Arguing as we did in the proof of Lemma B.5, we find that a similar estimate (sampled on the primal mesh) holds for $\overline{D q_{4}}$. We conclude with Corollary 3.8. 준

Collecting the estimates of $Q_{j}, j=1,2,3,4$, we have obtained in (B.12), (B.13), (B.17), and (B.20), we conclude the proof of Lemma 4.5.

B.5. Proof of Lemma 4.6. From the forms of $A_{2} v$ and $B_{2} v$ we have $I_{22}=$ $Q_{1}+Q_{2}$ with

$Q_{1}=-2 s \operatorname{Re} \iint_{Q} \xi_{1} r\left(\partial_{t}^{2} \rho\right)\left(\Delta_{\xi} \varphi\right)|v|^{2} d t, \quad$ and $Q_{2}=-2 s \operatorname{Re} \iint_{Q} \xi_{2} r(\bar{D} D \rho)\left(\Delta_{\xi} \varphi\right) \overline{\tilde{v}} v^{*} d t$.

By Lemma 3.4 we have $\overline{\tilde{v}}=v+h^{2} \bar{D} D v / 4$ which gives $Q_{2}=Q_{2}^{\prime}+Q_{2}^{\prime \prime}$ with

$$
\begin{aligned}
& Q_{2}^{\prime}=-2 s \operatorname{Re} \iint_{Q} \xi_{2} r(\bar{D} D \rho)\left(\Delta_{\xi} \varphi\right)|v|^{2} d t \\
& Q_{2}^{\prime \prime}=-\frac{s h^{2}}{2} \operatorname{Re} \iint_{Q} \xi_{2} r(\bar{D} D \rho)\left(\Delta_{\xi} \varphi\right)(\bar{D} D v) v^{*} d t .
\end{aligned}
$$

We first work on the expressions $Q_{1}$ and $Q_{2}^{\prime}$.

LEmma B.9. Provided $s h \leq \mathfrak{K}$ we have $\xi_{1} r \partial_{t}^{2} \rho=\xi_{1} s^{2} \lambda^{2}\left(\partial_{t} \psi\right)^{2} \varphi^{2}+s \mathcal{O}_{\lambda}(1)$ and $\left.\xi_{2} r(\bar{D} D \rho)=\xi_{2}\left(r \partial_{x}^{2} \rho+s^{2} \mathcal{O}_{\lambda, \mathfrak{K}}\left((s h)^{2}\right)\right)=\xi_{2}(s \lambda \varphi)^{2}\left(\partial_{x} \psi\right)^{2}+s \mathcal{O}_{\lambda}(1)+s^{2} \mathcal{O}_{\lambda, \mathfrak{K}}\left((s h)^{2}\right)\right)$.

The proof follows by Proposition 3.9 and Lemma 3.7.

Using (B.11), we have $Q_{1}+Q_{2}^{\prime}=-\iint_{Q} \mu|v|^{2} d t$ with

$$
\begin{aligned}
\mu & =2 s\left(s^{2} \lambda^{2}\left|\nabla_{\xi} \psi\right|^{2} \varphi^{2}+s \mathcal{O}_{\lambda}(1)+s^{2} \mathcal{O}_{\lambda, \mathfrak{K}}\left((s h)^{2}\right)\right)\left(\lambda^{2}\left|\nabla_{\xi} \psi\right|^{2} \varphi+\lambda \varphi \mathcal{O}(1)\right) \\
& =2 s^{3} \lambda^{4}\left|\nabla_{\xi} \psi\right|^{4} \varphi^{3}+s^{3} \lambda^{3} \varphi^{3} \mathcal{O}(1)+s^{2} \mathcal{O}_{\lambda}(1)+s^{3} \mathcal{O}_{\lambda, \mathfrak{K}}\left((s h)^{2}\right) .
\end{aligned}
$$

We now turn to the term $Q_{2}^{\prime \prime}$. For concision we set $q:=r \xi_{2}(D \bar{D} \rho)\left(\Delta_{\xi} \varphi\right)$. Since $v^{\partial \mathfrak{M}}=0$, discrete integrations by parts give

$$
\begin{aligned}
Q_{2}^{\prime \prime} & =-\frac{s h^{2}}{2} \operatorname{Re} \iint_{Q} q(\bar{D} D v) v^{*} d t=\frac{s h^{2}}{2} \iint_{Q} \tilde{q}|D v|^{2} d t+\frac{s h^{2}}{2} \operatorname{Re} \iint_{Q}(D q) \tilde{v}^{*} D v d t \\
& =\frac{s h^{2}}{2} \iint_{Q} \tilde{q}|D v|^{2} d t-\frac{s h^{2}}{4} \iint_{Q}(\bar{D} D q)|v|^{2} d t .
\end{aligned}
$$


We have $\Delta_{\xi} \varphi=\mathcal{O}_{\lambda}(1)$ and thus from Lemma B.9 we have $q=s^{2} \mathcal{O}_{\lambda, \mathfrak{K}}(1)$. The same estimate naturally holds for $\tilde{q}$. With the following lemma we conclude the proof.

Lemma B.10. Provided $s h \leq \mathfrak{K}$, we have $h^{2} \bar{D} D q=s(s h) \mathcal{O}_{\lambda, \mathfrak{K}}(1)$.

Proof. We set $p=\xi_{2}\left(\Delta_{\xi} \varphi\right)$ and observe that $\|p\|_{\infty}=\mathcal{O}_{\lambda}(1),\|D p\|_{\infty}=\mathcal{O}_{\lambda}(1)$, and $\|h \bar{D} D p\|_{\infty}=\mathcal{O}_{\lambda}(1)$. We thus have

$$
\begin{aligned}
h^{2} \bar{D} D q & =h^{2}(\bar{D} D p) \overline{\widetilde{r \bar{D} D \rho}}+2 h^{2} \overline{D p} \overline{\overline{D(r \bar{D} D \rho)}}+h^{2} \overline{\tilde{p}}(\bar{D} D(r \bar{D} D \rho)) \\
& =\left(h+h^{2}\right) s^{2} \mathcal{O}_{\lambda, \mathfrak{K}}(1),
\end{aligned}
$$

by Propositions 3.9 and 3.12 .

B.6. Proof of Lemma 4.7. We have $W=\iint_{Q} p\left|D \partial_{t} v\right|^{2} d t$ with

$$
p=\frac{1}{2} h^{2} s \lambda^{2}\left(\xi_{1} \varphi\left|\nabla_{\xi} \psi\right|^{2}\right)_{d}+h^{2} s \lambda \varphi_{d} \mathcal{O}(1)+h \mathcal{O}(s h)+h \mathcal{O}_{\lambda, \mathfrak{K}}\left((s h)^{2}\right) .
$$

Since $\left|\nabla_{\xi} \psi\right| \geq C>0$, we see that for $\lambda$ sufficiently large, the first term above dominates the second and third terms for any $h, s$, so that we obtain $p \geq h^{2} s\left(C-C^{\prime} s h\right)$ and thus $W \geq 0$ for $s h$ sufficiently small. Next, since $\operatorname{reg}(\xi) \leq \operatorname{reg}^{0}$, we see that

$$
Y=\int_{0}^{T_{*}}\left(q_{N+1}|D v|_{N+\frac{1}{2}}^{2}-q_{0}|D v|_{\frac{1}{2}}^{2}\right) d t, \quad \text { with } \quad q=\left(1+\mathcal{O}_{\lambda, \mathfrak{K}}\left((s h)^{2}\right)\right) \xi_{2} \overline{\xi_{2}} r \overline{D \rho} .
$$

By (4.1) we have $Y \geq 0$ for $s h$ sufficiently small.

B.7. Proof of Lemma 4.9. By Lemma 3.2 we have $r_{d} D u=\tilde{v} r_{d} D \rho+r_{d} \tilde{\rho} D v$, which by Proposition 3.9, yields

$$
\left|r_{d} D u\right|_{L^{2}(\Omega)}^{2} \leq C_{\lambda, \mathfrak{K}}\left(\left|\tilde{v} r_{d} D \rho\right|_{L^{2}(\Omega)}^{2}+|D v|_{L^{2}(\Omega)}^{2}\right),
$$

We observe that

$$
\left|\tilde{v} r_{r} D \rho\right|_{L^{2}(\Omega)}^{2}=\int_{\Omega}|\tilde{v}|^{2}\left(r_{d} D \rho\right)^{2} \leq \int_{\Omega} \widetilde{|v|^{2}}\left(r_{d} D \rho\right)^{2}=\int_{\Omega}|v|^{2} \overline{\left(r_{d} D \rho\right)^{2}}=s^{2} \int_{\Omega} \mathcal{O}_{\lambda, \mathfrak{K}}(1)|v|^{2},
$$

since $v^{\partial \mathfrak{M}}=0$ and by Proposition 3.9, which yields the first result.

The proof of the second result is similar, yet simpler. We have $r \partial_{t} u=\partial_{t} v+$ $r\left(\partial_{t} \rho\right) u$, which implies

$$
\left|r \partial_{t} u\right|_{L^{2}(\Omega)}^{2} \leq C_{\lambda, \mathfrak{K}}\left(\left|\partial_{t} v\right|_{L^{2}(\Omega)}^{2}+s^{2}|v|_{L^{2}(\Omega)}^{2}\right) .
$$

The last result follows the same.

Appendix C. On the construction of the Carleman weight function. We describe here the succession of arguments used in the construction of the Carleman weight function $\psi$. Its regularity class is $\mathscr{C}^{k}(\overline{\tilde{Q}})$ for a certain $k \in \mathbb{N}$ prescribed in advance. Note however that the set $\Omega$ itself needs to be of class $\mathscr{C}^{k}$.

We first start with a function $\phi_{1}(t) \in \mathscr{C}^{\infty}\left(\left[0, T_{*}\right]\right)$ such that $\partial_{t} \phi_{1}(0) \geq C>0$, $\partial_{t} \phi_{1}\left(T_{*}\right) \leq-C<0$, and $\phi_{1}(0)=\phi_{1}\left(T_{*}\right)=0$, and $\phi_{1}(t)>0$ if $t \in\left(0, T_{*}\right)$. We also choose $\phi_{2}(x) \in \mathscr{C}^{k}(\bar{\Omega})$ such that $\phi_{2} \geq C>0$ and $\partial_{n_{x}} \phi_{2} \leq-C^{\prime}<0$ in $V_{\partial \Omega}$, which can be achieved by choosing the neighborhood $V_{\partial \Omega}$ sufficiently small. We next set $\phi(t, x)=\phi_{1}(t) \phi_{2}(x)$. This function satisfies the desired properties listed in 
Assumption 2.1 on the boundaries $\left(0, T_{*}\right) \times \partial \Omega$ (and in its neighborhood $\left.\left(0, T_{*}\right) \times V_{\partial \Omega}\right)$, $\{0\} \times(\Omega \backslash \omega)$ and $\left\{T_{*}\right\} \times \Omega$.

We choose $y_{0}$ in $\{0\} \times \omega$. We enlarge $Q$ in a small neighborhood of $y_{0}$ which leaves $\partial Q$ unchanged outside of $\{0\} \times \omega$. We call $\mathcal{Q}$ this extension of $Q$ and we extend the function $\phi$ to $\mathcal{Q}$ in a $\mathscr{C}^{k}$ manner.

The function $\phi$ exhibits only one critical point points in $Q$. It can be pulled back to the interior of $\mathcal{Q} \backslash Q$ by composing $\phi$ with a diffeomorphism (see [FI96] for the construction of such a diffeomorphism). The resulting function is the weight function $\psi$ and it satisfies all the properties listed in Assumption 2.1.

Acknowledgments: The authors thank Assia Benabdallah for discussions on the subject of the present article.

\section{REFERENCES}

[AE84] J.-P. Aubin and I. Ekeland, Applied Non Linear Analysis, John Wiley \& Sons, New York, 1984

[Bar00] V. Barbu, Exact controllability of the superlinear heat equation, Appl. Math. Optim. 42 (2000), 73-89.

[BHL09a] F. Boyer, F. Hubert, and J. Le Rousseau, Discrete carleman estimates for elliptic operators in arbitrary dimension and applications, In prep. (2009).

[BHL09b] F. Boyer, F. Hubert, and J. Le Rousseau, On the approximation of the nullcontrollability problem for parabolic equations, Algoritmy09, 18th Conference on Scientific Computing (Slovak University of Technology, ed.), 2009.

[BHL09c] F. Boyer, F. Hubert, and J. Le Rousseau, Uniform null-controllability properties for space/time-discretized parabolic equations, Preprint, http://hal.archives-ouvertes.fr/hal-00429197/fr/ (2009).

[FCZ00] E. Fernández-Cara and E. Zuazua, Null and approximate controllability for weakly blowing up semilinear heat equations, Ann. Inst. H. Poincaré, Analyse non lin. 17 (2000), 583-616.

[FI96] A. Fursikov and O. Yu. Imanuvilov, Controllability of evolution equations, vol. 34, Seoul National University, Korea, 1996, Lecture notes.

[GL94] R. Glowinski and J.-L. Lions, Exact and approximate controllability for distributed parameter systems, Acta Numer., Cambridge Univ. Press, Cambridge, 1994, pp. 269378.

[JL99] D. Jerison and G. Lebeau, Harmonic analysis and partial differential equations (Chicago, IL, 1996), Chicago Lectures in Mathematics, ch. Nodal sets of sums of eigenfunctions, pp. 223-239, The University of Chicago Press, Chicago, 1999.

[KS91] M. V. Klibanov and F. Santosa, A computational quasi-reversibility method for Cauchy problems for Laplace's equation, SIAM J. Appl. Math. 51 (1991), 1653-1675.

[Le 07] J. Le Rousseau, Représentation Microlocale de Solutions de Systèmes Hyperboliques, Application à l'Imagerie, et Contributions au Contrôle et aux Problèmes Inverses pour des équations Paraboliques, Mémoire d'habilitation à diriger des recherches, Universités d'Aix-Marseille, Université de Provence, 2007, http://tel.archives-ouvertes.fr/tel-00201887/fr/.

[LL09] J. Le Rousseau and G. Lebeau, On Carleman estimates for elliptic and parabolic operators. applications to unique continuation and control of parabolic equations, Preprint (2009).

[LR95] G. Lebeau and L. Robbiano, Contrôle exact de l'équation de la chaleur, Comm. Partial Differential Equations 20 (1995), 335-356.

[LT06] S. Labbé and E. Trélat, Uniform controllability of semidiscrete approximations of parabolic control systems, Systems Control Lett. 55 (2006), 597-609.

[LZ98a] G. Lebeau and E. Zuazua, Null-controllability of a system of linear thermoelasticity, Arch. Rational Mech. Anal. 141 (1998), 297-329.

[LZ98b] A. Lopez and E. Zuazua, Some new results to the null controllability of the 1-d heat equation, Sminaire sur les quations aux Drives Partielles, 1997-1998, Exp. No. VIII, 22 pp., cole Polytech., Palaiseau (1998).

[Mil06] L. Miller, On the controllability of anomalous diffusions generated by the fractional laplacian, Mathematics of Control, Signals, and Systems 3 (2006), 260-271. 
[Zhe08] C. Zheng, Controllability of the time discrete heat equation, Asymptotic Analysis 59 (2008), 139-177.

[Zua06] E. Zuazua, Control and numerical approximation of the wave and heat equations, International Congress of Mathematicians, Madrid, Spain III (2006), 1389-1417. 